\title{
1 Retracing the evolution from antimicrobial to targeting
}

2 peptides

3

4 Caspari, O.D..$^{1,2 *}$, Garrido, C. ${ }^{1}$, Choquet, Y. ${ }^{1}$, Wollman F.-A. ${ }^{1}$, Lafontaine, $\mathrm{I}^{1 *}$

5

6 Affiliations :

7 Institut de Biologie Physico-Chimique (CNRS/Sorbonne Université), 13 Rue

8 Pierre et Marie Curie, 75005 Paris, France

9

10 Current address :

11 2Department of Microbiology, Institut Pasteur, 28 Rue Docteur Roux, 75015

12 Paris, France

13

$14 *$ Correspondance :

15 ODC : odc20@cantab.net

16 IL : ingrid.lafontaine@ibpc.fr

17

18 Keywords : Evolution, endosymbiosis, organellogenesis, chloroplast,

19 mitochondria, subcellular targeting, protein import, transit peptide,

20 presequence, Chlamydomonas reinhardtii 


\section{Abstract}

22 We experimentally challenged the endosymbiotic hypothesis that organelle-

23 targeting peptides derive from antimicrobial amphipathic peptides delivered by

24 the host cell, to which organelle progenitors became resistant. To explore the

25 molecular changes required to convert such antimicrobial peptides into bona fide

26 organelle-targeting peptides, we expressed a set of 13 antimicrobial peptides of

27 various origins in the green alga Chlamydomonas reinhardtii that serves as a

28 model for both mitochondrial and chloroplast import. The peptides were

29 modified to match distinctive features of mitochondrial and chloroplast targeting

30 peptides, and we assessed their targeting potential by following the intracellular

31 localization and maturation of a Venus fluorescent reporter used as cargo

32 protein. We present a temporal evolutionary scenario that emphasizes the early

33 contribution of exchanging Lysines with Arginines in the sequence of the

34 antimicrobial peptide, the evolution of a processing site followed by the addition

35 of unstructured sequence and protein interaction sites that allow the selective

36 targeting to the chloroplast.

\section{Introduction}

38 Antimicrobial peptides (AMPs) have recently been proposed to be at the

39 evolutionary origin of N-terminal targeting peptides (TPs) that direct proteins

40 into mitochondria and chloroplasts ${ }^{1,2}$. Part of the innate immune system, AMPs

41 are produced by virtually all types of cells in a bid to kill or control microbial

42 adversaries ${ }^{3,4}$. The proposed evolutionary scenario posits that early during

43 endosymbiosis, the host used AMPs to attack the bacterial proto-endosymbiont. 
44 A bacterial resistance mechanism, whereby the AMP is imported into the

45 bacterial cytoplasm, would have generated a pathway for host proteins to reach

46 the bacterial cytosol - a plausible first step in the evolution of a protein import

47 machinery. Cationic, Helical-Amphipathic Ribosomally-produced AMPs (HA-

48 RAMPs) and TPs share key physico-chemical properties and they were shown, in

49 some instances, to retain cross-functionality5: several TPs showed antimicrobial

50 activity and five HA-RAMPs, fused to a cleavage-site containing TP element, were

51 shown to promote the import of a Venus fluorescent protein into either the

52 mitochondria or the chloroplast of the model green alga Chlamydomonas

53 reinhardtii.

54 The main part of mTPs and the central element of cTPs appear derived from HA-

55 RAMPs on account of a shared cationic, amphipathic helical structure that often

56 encompasses the entire length of the HA-RAMPs ${ }^{2}$ and mTPs ${ }^{6,7}$. Although plant

57 cTPs have been described as unstructured ${ }^{8}$, algal cTPs more closely resemble

58 mTPs in being helical ${ }^{9}$. Helices were observed by NMR in membrane-mimetic

59 environments in both algal and plant CTPs $^{10-14}$, and signatures of amphipathic

60 helices can be detected in a majority of cTPs ${ }^{5}$.

61 In addition to the helices, both mTPs and cTPs contain recognition sites at the C-

62 terminus where processing peptidases in the mitochondrial matrix (MPP) and

63 the chloroplast stroma (SPP) cleave off the cargo protein ${ }^{15}$. These recognition

64 sites encompass some 10 residues upstream of the cleavage site and are

65 structurally distinct from the rest of the TPs, even showing a weak sequence

66 conservation $6,16,17$. While targeting information is usually contained within mTP

67 sequences upstream of the cleavage site, targeting by cTPs shorter than ca. 60

68 amino acids often requires downstream unstructured sequence stretches in the 
$\mathrm{N}$-terminal domain of the mature protein ${ }^{18,19}$. Besides the amphipathic helical module and the C-terminal cleavage module shared between mTPs et cTPs, it has been argued that distinct features at their N-termini conferred organelle specificity to each set of targeting peptides $6,16,20-26$.

In this study, we systematically introduced modifications into candidate HARAMPs to retrace likely steps along the evolution of TPs that improved targeting ${ }^{1,2}$. We thus retrace the evolutionary switches from HA-RAMPs to TPs by adding $\mathrm{N}$ - and $\mathrm{C}$-terminal TP elements to $13 \mathrm{HA}$-RAMP sequences, chosen for their similarity to TPs, in a bid to generate targeting to the mitochondria or chloroplast in the model green alga Chlamydomonas reinhardtii. Besides providing a guide to the most likely evolutionary sequence of events, this dataset also provides new insights into how different $\mathrm{TP}$ elements contribute to differential targeting and to the efficiency of protein import. We show that some HA-RAMPs natively contain such sequence elements, with some HA-RAMPs being prone to chloroplast targeting while others show a preference for the mitochondria. Furthermore, we provide evidence for a critical evolutionary contribution of a substitution of cationic residues from Lysine (K) in HA-RAMPs to Arginine (R) in TPs.

\section{Results}

Figure 1 shows the major sequence features of the 13 HA-RAMPs used in the present study (Fig. 1A) together with those of a typical cTP and a typical mTP (Fig. 1B). On the right-hand side, these peptides are represented according to their proportion of $\alpha$-helical amphipathic structure. Three HA-RAMPs (Magainin II, Brevinin 1B and Dermaseptin S4) were chosen based on a manual comparison 
with TP sequences, and a further ten were chosen based on scoring as highly

94 similar to TPs in a recent analysis ${ }^{5}$, such that different AMP families would be

95 represented.

96 To explore targeting, the 13 HA-RAMPs were systematically modified (Fig. 2),

97 notably by adding non-helical TP elements from the $\mathrm{N}$ - and/or C-terminal

98 domains of Chlamydomonas Rubisco activase (RBCA) cTP, or a C-terminal

99 domain of similar length from mitochondrial $\gamma$-carbonic anhydrase 2 (CAG2)

100 mTP (Fig. 1B, Fig. 2). Peptides carrying the 15 amino-acid long cTP N-terminus

101 (cTP-N) will be denoted cP. Similarly, peptides with C-terminal elements from

102 RBCA-cTP (cTP-C) or CAG2-mTP (mTP-C) will be denoted $\mathrm{P}^{\mathrm{c}}$ or $\mathrm{P}^{\mathrm{m}}$ respectively.

103 Micrographs for three biological replicates per construct are displayed on Sup.

104 Figs. 1-18.

105 In the absence of any modifications, only Brevinin-2I (B2I) and Magainin 2 (MII)

106 out of the set 13 HA-RAMPs were capable of organelle-targeting visible in

107 fluorescence microscopy (Fig. 2A). When equipped with B2I, the fluorescent

108 reporter Venus shows mitochondrial localization: the Venus signal is observed as

109 a characteristic network pattern that co-localizes with MitoTracker fluorescence

110 (Fig. 3). By contrast, in the case of MII only a small fraction of fluorescence signal

111 in the Venus channel shows co-localisation with the mitotracker dye, signifying

112 that targeting is only partial (Fig. 3).

\section{$113 \mathrm{~K} / \mathrm{R}$ content contributes to functional divergence between HA-RAMP and TP}

114 We looked for differences in primary sequence that might impede the ability of

115 the remaining 11 HA-RAMPs to target. As illustrated on Supp. Fig. 19, extant HA-

116 RAMPs and organellar TPs display a few differences in their amino-acid content. 
117 HA-RAMPs are richer in helix-breaking Glycines (G) but poorer in Prolines (P),

118 whereas TPs are enriched in Serines (S) compared to HA-RAMPs. Most of the

119 other amino acid frequencies are broadly similar between the two sets of 120 peptides and within range of the uniprot average. As expected, both HA-RAMPs

121 and TPs are poor in acidic residues (D, E) but enriched in basic residues (K or R).

122 However, their complement in basic residues is markedly different (Fig. 4A):

123 HA-RAMPs are rich in Lysines (K) whereas TPs are rich in Arginines (R).

124 In order to see whether these contrasting differences in K/R ratio contributed to

125 the functional divergence between HA-RAMPs and TPs, we substituted all

126 instances of $\mathrm{R}$ by $\mathrm{K}$ in TPs and of $\mathrm{K}$ by $\mathrm{R}$ in HA-RAMPs. In the rest of the text, the

127 basic amino acid mostly present in a given peptide $\mathrm{P}$ will be indicated by a

128 subscript as $\mathrm{P}_{\mathrm{R}}$ or $\mathrm{P}_{\mathrm{K}}$. HA-RAMPs with $\mathrm{K} \rightarrow \mathrm{R}$ transition showed reduced

129 antimicrobial activity, as illustrated on Fig. 4B by the increased minimum

130 inhibitory concentrations for MII, Dermaseptin S4 (DS4) or Brevinin 1E (B1E).

131 Conversely, when the natively R-rich Chlamydomonas Rubisco activase (RBCA)

132 cTP $\left({ }^{\mathrm{C}} \mathrm{CH}_{\mathrm{R}} \mathrm{c}\right)$ and mitochondrial $\gamma$-carbonic anhydrase 2 (CAG2) mTP $\left(\mathrm{MH}_{\mathrm{R}}{ }^{\mathrm{m}}\right)$

133 underwent systematic $\mathrm{R} \rightarrow \mathrm{K}$ substitutions $\left({ }^{\mathrm{c}} \mathrm{CH}_{\mathrm{K}}^{\mathrm{c}}, \mathrm{MH}_{\mathrm{K}} \mathrm{m}\right)$, their ability to target

134 the chloroplast and the mitochondria were reduced or abolished (Fig. 4C). Note

135 that TP helical fragments, devoid of N- and C-terminal domains, are denoted as

$136 \mathrm{MH}$ for mTP and $\mathrm{CH}$ for cTP (Fig. 1B). These experiments demonstrate the

137 respective functional contributions of $\mathrm{K}$ and $\mathrm{R}$ residues to antimicrobial and

138 organelle targeting activity. We then systematically re-examined the organelle

139 targeting ability of the set of 13 HA-RAMP that had undergone $\mathrm{K} \rightarrow \mathrm{R}$

140 substitutions (Fig. 2B). $\mathrm{MII}_{\mathrm{R}}$ now shows full mitochondrial targeting, and $\mathrm{R} 2 \mathrm{G}_{\mathrm{R}}$ 
141 (Ranatuerin 2G) as well as $\mathrm{CP}_{\mathrm{R}}$ (Cecropin $\mathrm{P} 3$ ) show partial targeting, as

142 evidenced by significant overlap of Venus and mitotracker fluorescence (Fig. 4D).

\section{Targeting peptide elements contribute to targeting}

\section{$144 \quad$ C-termini matter for targeting}

145 Addition of mTP-C, the cleavage-site containing C-terminal element of CAG2-

146 mTP (Fig. 1B) enabled mitochondrial targeting for ES1 $1_{\mathrm{R}}$ (Esculentin $1 \mathrm{~S}$ ) and

$147 \quad \mathrm{R} 2 \mathrm{G}_{\mathrm{K}}$ and improved the targeting already observed for $\mathrm{R} 2 \mathrm{G}_{\mathrm{R}}$ and $\mathrm{CP} 3_{\mathrm{R}}$, in 148 addition to continued targeting by B2I and MII (Fig. 2CD). The impact of adding 149 cTP-C, the C-terminal element derived from RBCA-cTP (Fig. 1B) is even more 150 important: cTP-C significantly enabled chloroplast targeting (Fig. 2E,F, Fig. 5B),

151 which could be seen in 9 HA-RAMP constructs involving EHF (Enterocin HF), 152 B15 (Bacillocin 1580), LCA (Leucocin A), S1D (Sarcotoxin 1D) and CP3 $3_{R}$. The 153 addition of cTP-C to the helical part of the CAG2-mTP $\left(\mathrm{MH}_{\mathrm{R}}{ }^{\mathrm{c}}\right)$ even partially 154 retargets the construct to the chloroplast (Fig. 5C), and deletion of cTP-C 155 abolishes chloroplast targeting by RBCA-cTP $\left({ }^{\mathrm{c}} \mathrm{CH}_{\mathrm{R}}\right)$. By comparison, deletion of 156 mTP-C reduces but does not totally abolish mitochondrial targeting of CAG2157 mTP $\left(\mathrm{MH}_{\mathrm{R}}\right)$, and addition of mTP-C fails to rescue ${ }^{\mathrm{c}} \mathrm{CH}_{\mathrm{R}}\left({ }^{\mathrm{c}} \mathrm{CH}_{\mathrm{R}}{ }^{\mathrm{m}}\right)$. Note that the 158 addition of cTP-C was also compatible with mitochondrial localization by B2I 159 and MII and the addition of MTP-C also enabled chloroplast localization by EHF $_{\mathrm{R}}$ 160 (Fig. 2D-F, Fig. 5A,B).

\section{$161 \quad N$-termini matter for chloroplast targeting}

162 The sole addition of cTP-N generated at least partial chloroplast targeting (Fig. 163 2G,H, Fig. 5D) in 6 HA-RAMP constructs, notably by SI Moricin (SIM) as well as $164 \mathrm{EHF}_{\mathrm{R}}, \mathrm{B} 15_{\mathrm{R}}, \mathrm{E} 1 \mathrm{~S}_{\mathrm{R}}$ and $\mathrm{CP} 3_{\mathrm{R}}$. However, cTP-N also enabled mitochondrial 
165

166

167

168

169

170

171

172

173

174

175

176

177

178

180

181

182

183

184

185

186

187

targeting (Fig. 2G,H, Fig. 5E) in three HA-RAMPs that had not previously shown mitochondrial localization, B1E, S1D and $\mathrm{DDM}_{\mathrm{R}}$ (Dermadistinctin M).

The importance of cTP-N as a chloroplast determinant becomes most evident when combined with a C-terminal element: addition of cTP-N to constructs equipped with mTP-C (Fig. 2I,J) resulted in 10 HA-RAMP constructs targeting the chloroplast (EHF, B15, SIM, E1S, LCA, CP3 $3_{\mathrm{K}}$ ) as well as 6 constructs showing dual targeting to the chloroplast and the mitochondria (S1D, B1E, MII,$B 2 I_{R}$ ). Combining cTP-N with cTP-C (Fig. 2KL) generates at least partial chloroplast targeting in 22 out of 26 HA-RAMP constructs (including 1 instance of dual targeting by $\mathrm{R}_{2} \mathrm{G}_{\mathrm{R}}$ ); only Dermaseptin S4 (DS4) fails to show any chloroplast targeting. Consider B2I with cTP-N (cB2I, Fig. 2G-La) as an example of the effect of combining cTP-N with C-termini, shown in Fig. 5F. Equipped with mTP-C, ${ }^{\mathrm{c}} \mathrm{B} 2 \mathrm{I}_{\mathrm{K}}{ }^{\mathrm{m}}$ targets the mitochondria whereas addition of $\mathrm{cTP}-\mathrm{C}\left({ }^{\mathrm{c}} \mathrm{B} 2 \mathrm{I}_{\mathrm{K}}{ }^{\mathrm{c}}\right)$ generates partial targeting to the chloroplast. Upon $\mathrm{K} \rightarrow \mathrm{R}$, the presence of $\mathrm{mTP}-\mathrm{C}\left({ }^{\mathrm{c} B} 2 \mathrm{I}_{\mathrm{R}}^{\mathrm{m}}\right)$ now supports dual targeting and full chloroplast targeting is observed in the presence of cTP-C $\left({ }^{c} B 2 I_{R}{ }^{c}\right)$.

Finally, Fig. 5G shows that while addition of cTP-N to the CAG2-mTP abolished mitochondrial targeting $\left({ }^{\mathrm{c} M H_{\mathrm{R}}} \mathrm{m}\right)$, the $\mathrm{cTP}-\mathrm{N} / \mathrm{cTP}-\mathrm{C}$ combination also retargets $\mathrm{MH}$ to the chloroplast, and enables partial chloroplast targeting of a control in which the central element is composed of a poly-Alanine tract $\left({ }^{c} A A^{c}\right)$ devoid of amphipathic properties and of a no peptide control $\left(^{c_{-}}\right)^{c}$. The two latter experiments demonstrate that there are enough determinants for recognition of the chloroplast translocon dispersed between the Nter and Cter of a cTP, to target Venus to the chloroplast, albeit with a lower efficiency than when an amphipathic helix is present in between. 
190

191 silico comparisons of the studied peptides. In Principal Component Analyses (on

192 ACC Z-scales ${ }^{5}$ ) comparing TP N- and C-termini with our 13 HA-RAMPs (Sup. Fig.

193 20), cTPs and mTPs could be differentiated much better by N- compared to C-

194 termini, supporting N-termini as important specificity determinants.

195 Furthermore, even though algal cTP N-termini are more charged, less 196 hydrophobic and more disordered than higher plant cTPs (Sup. Fig. 21) (in line 197 with algal cTPs being more mTP-like ${ }^{9}$ ), Chlamydomonas cTP N-termini are 198 recognized as chloroplast-targeting in a model trained on Arabidopsis TP and 199 vice versa (Sup. Fig. 21), confirming that essential physico-chemical features of 200 chloroplast determination in CTP N-termini are maintained in both lineages.

\section{HA-RAMPs dominate targeting specificity}

202 Considering Fig. 2 by columns reveals that organelle specificity is, to a large 203 extent, determined by HA-RAMPs. Only 3 HA-RAMPs (B2I, R2G, MII; referred to 204 hereafter as the mt-set) account for $>70 \%$ of all constructs where mitochondrial 205 targeting is seen. Similarly, 5 HA-RAMPs (E1S, LCA, SIM, B15, EHF; referred to as 206 the cp-set) account for the majority ( $\sim 57 \%)$ of all chloroplast targeting, and for 207 two thirds when excluding the cTP-N/cTP-C combination that generates 208 chloroplast targeting across most HA-RAMPs. In some instances, a set of peptide 209 modifications may switch targeting from the mitochondria to the chloroplast, yet 210 the major effect of modifications, i.e. exchanging $K \rightarrow$ R (Fig. 4, Sup. Fig. 22) or 211 adding TP elements (Fig. 5, 6), is to enhance the targeting ability to an organelle 212 determined by the HA-RAMP primary sequence properties (Fig. 2). 
214 To understand what differentiates mt-set from cp-set HA-RAMPs, we examined

215 the sequence characteristics of our 13 HA-RAMPs with those of well-

216 characterized Chlamydomonas targeting peptides (Fig. 6). In Chlamydomonas,

217 mTPs are ca. 35 residues in length (Fig. 6A). By contrast, most cTPs are

218 significantly longer $(\mathrm{p}=0.0017)$. The difference is even greater than shown here

219 given that many cTPs require a contribution from post-cleavage site residues for

220 successful targeting5,18,19. Consistently, cp-set HA-RAMPs (in green, Fig. 6A) are

221 longer than mt-set HA-RAMPs (in orange; $\mathrm{p}=0.0184$ ) and require further

222 elongation by addition of TP elements before targeting can be observed (Fig. 2).

223 Most mTPs fold into an amphipathic helix for most of the sequence $(\sim 80 \%$ on

224 average, Fig. 6B) starting directly from their N-terminus (Fig. 6C). The fraction

225 dedicated to amphipathic helix formation in cTPs is significantly lower (Fig. 6B,

$226 \mathrm{p}<0.0001$ ), with a longer stretch of non-helical sequence at the N-terminus (Fig.

227 6C, $\mathrm{p}<0.0001)$. A PCA analysis based on these properties, plus the fraction of $\mathrm{R}$

228 and K, recapitulates the combined properties within each peptide (Sup. Fig. 23).

229 The PCA shows that cp-set HA-RAMPs are localized with cTPs in the upper left

230 area of the graph, consistent with a lower helix fraction (Fig. 1, Fig. 6B) due to

231 longer sequence stretches upstream of the main helix than in the mt-set HA-

232 RAMPs (Fig. 1, Fig. 6C, p=0.0199). Consistent with the importance of these

233 helical features, LCA, the most helical among cp-set HA-RAMPs also shows the

234 highest fraction of only partial chloroplast targeting (3 out of 5 targeting 235 constructs, Fig. 2j rows E,F,K). Furthermore, SIM and E1S, the two cp-set HA-

236 RAMPs with the shortest pre-helix segments (Fig7C), both require addition of 237 cTP-N for chloroplast targeting (Fig. 2i,k). 
cTPS are predicted to be more prone to protein interaction than mTPS

239 cTPs appear more likely to interact with proteins than mTPs (Sup. Fig. 24), just

240 like cp-set HA-RAMPs. 'FGLK' motifs that are reportedly TOC interaction sites in

241 plant cTPs $^{20}$ appear shortened to 'GLK' motifs in Chlamydomonas but they are

242 associated with an increased protein interaction potential in cTPs compared to

243 mTPs $(\mathrm{p}<0.0001)$, including within cTP-N and cTP-C elements. However, Hsp70-

244 interaction sites ${ }^{23}$, which are commonly found at cTP N-termini ${ }^{20}$, also occur at a

245 high frequency in mTP sequences and HA-RAMPs (Sup. Fig. 24, 25).

247 To characterize the maturation of HA-RAMP-targeted proteins upon organellar

248 import, we performed immunoblotting experiments (Fig. 7). Indicative examples

249 were selected for clarity; a more comprehensive overview is provided in Sup.

$250 \quad$ Fig. 26.

251 In the absence of a dedicated cleavage site (Fig. 7A), we found some cleavage did 252 occur but preproteins are also maintained: two bands are observed in constructs 253 that were localized in an organelle by fluorescence imaging (lanes d,f-j). The 254 upper band corresponds to unprocessed Venus, which is a fusion of the reporter 255 with the HA-RAMP construct and migrates at varying positions depending on the 256 length of the presequence. The lower band corresponds to the processed form 257 migrating closer to the "free" Venus position (lane c) depending on the exact site 258 of cleavage. The cTP and mTP controls (lanes a,b) contain 23 residues between 259 cleavage site and Venus (Venus+23), and maintain very little preprotein 260 migrating above. In HA-RAMP constructs by contrast, the majority of Venus 261 remained in the unprocessed top band for constructs targeting the mitochondria 262 (lanes $\mathrm{f}-\mathrm{j}$ ). Nonetheless, the presence of a faint processed form in the lower band 
263 just above free Venus shows that some cleavage occurs. The proportion of

264 processed versus unprocessed Venus preprotein is much higher in partially

265 chloroplast-targeted ${ }^{\mathrm{c}} \mathrm{SIM}_{\mathrm{K}}$ (lane d), hinting at more efficient degradation of the

266 unprocessed form in the chloroplast (see also Sup. Fig. 26).

267 Addition of cleavage sites improves cleavage across organelles: In the presence

268 of either cTP-C (Fig. 7B, Blot 1) or mTP-C (Fig. 7B, Blot 2), which contain the

269 RBCA-cTP and CAG2-mTP cleavage sites respectively, targeting constructs

270 appear to be cleaved at a site corresponding to TP controls (Venus+23).

271 Constructs equipped with either of the two cleavage sites deliver a processed

272 Venus of similar size, independently of whether the mitochondria or the

273 chloroplast are targeted (Fig. 7B). These results indicate that mTP and cTP

274 cleavage sites are recognized in both organelles. We note that in the case of HA-

275 RAMP constructs equipped with mTP-C, cleavage appears to occur not at the

276 cleavage site previously identified for CAG2 (Fig. 7B Blot 2 lane a, top band,

277 corresponding to Venus+23), but instead at or near a second site closer to "free"

278 Venus (compare Fig. 7B Blot 2 lane a, bottom band to lanes b-g), implying

279 processing within ten residues upstream of Venus. Unlike mTP-C (Fig. 7B Blot 2

280 lane h), cTP-C appears to be processed in the absence of targeting (Fig. 7B Blot 1

281 lanes a,b) at a site different to the one used in organelles (Fig. 7B Blot 1 lanes c-

$282 \mathrm{~h})$.

283 A switch from $\mathrm{K}$ to $\mathrm{R}$ (Fig. 7C), which improved targeting, also increased the

284 amount of processed form relative to that of the unprocessed form in all cases,

285 whether due to a gain of targeting (lanes f-i) or an increase in efficiency (lanes b-

286 e), suggesting that cleavage can serve as a proxy for targeting. Indeed, there is a

287 remarkable correlation between which constructs show cleavage, as observed in 
288 immunoblots, and which constructs show targeting, as judged from microscopy

289 (Sup. Fig. 26). We note that faint processed bands can be seen for several 290 unmodified HA-RAMPs (Sup. Fig. 26), suggesting further peptides in addition to $291 \mathrm{~B}^{2} \mathrm{I}_{\mathrm{K}}$ and $\mathrm{MII}_{\mathrm{K}}$ (Fig. 2) may be able to support some amount of targeting that is, 292 however, too partial to be seen in fluorescence microscopy.

\section{Discussion}

\section{How TPs may have evolved from HA-RAMPs}

295 In an assessment of the hypothesis that extant TPs are derived from a subclass of 296 antimicrobial peptides that harbor an amphipathic helix, the HA-RAMPs ${ }^{1,2}$, we 297 studied a systematic series of HA-RAMP constructs. This dataset allows us to 298 propose an evolutionary scenario (Fig. 8) for the conversion of such peptides 299 from antimicrobial to bona fide targeting peptides whose properties allow 300 differential targeting between mitochondria and chloroplasts. We showed that a $301 \mathrm{~K}$ to R switch reduces cytotoxicity of HA-RAMPs, while increasing targeting. This 302 dual effect must produce a strong selection pressure favoring this exchange at an 303 early step of the endosymbiosis process (Fig. 8A). The underlying mechanism 304 warrants further research, but the finding is in line with the fact that Arginines 305 provide a better uptake efficiency than other cationic residues because of the 306 guanidinium group forming bidentate hydrogen bonds with membrane 307 phosphate groups ${ }^{27,28}$. Differences in bulkiness or in $\mathrm{pKa}^{29,30}$, or trans-acting 308 factors such as regulation of targeting through ubiquination of $\mathrm{K}^{31}$, may also play 309 a role. Mitochondria appear able to tolerate the presence of HA-RAMP310 containing preproteins, suggesting mTPs could have evolved relatively easily 
311 from HA-RAMPs. Even unaltered HA-RAMPs can in some cases deliver cargo,

312 providing a plausible starting point for protein import. Thus addition of cleavage

313 sites would likely have come as a second step (Fig. 8B), allowing presequences to

314 be degraded separately from the cargo protein ${ }^{32}$. Interestingly, Arginines feature

315 prominently in Chlamydomonas cleavage site consensus sequences ${ }^{17}$, thus

316 cleavage sites may be generated by the $\mathrm{K} \rightarrow \mathrm{R}$ switch, potentially accounting for

317 increased chloroplast-localization among cTP-N bearing constructs (Fig. 2GH).

318 Since cTPs evolved in a cell that already had mTPs, additional discriminating

319 elements were needed to avoid large scale mistargeting. We found that $\mathrm{N}$ -

320 terminal elements interact with suitable C-termini to determine chloroplast

321 targeting. The $15 \mathrm{~N}$-terminal residues have been suggested as specificity

322 determinants in the literature before ${ }^{20-22}$, however, the contribution of this

323 element to chloroplast targeting has remained under debate. Our data suggests

324 that rather than providing an absence of Arginines ${ }^{21,33}$ (Sup. Fig. 21) or a

325 difference in Hsp70 binding $\operatorname{sites}^{20,25,26}$ (Sup. Fig. 24), cTP N-termini appear to

326 contribute unstructured sequence upstream of the amphipathic helix. Thus HA-

327 RAMPs that intrinsically contained relatively unstructured N-termini proved

328 most able to support chloroplast targeting even in the absence of cTP-N. The

329 need to contain unstructured sequence ${ }^{8}$ as well as the increased length of cTPs,

330 even extending beyond the cleavage site in many cases ${ }^{5,18,19}$, appear

331 mechanistically related to the import system. Whereas mitochondrial import

332 makes use of the proton gradient across the inner membrane to power uptake of

333 positively charged presequences ${ }^{34,35}$, energized chloroplast import requires the

334 cTP to be elongated enough to reach across both TOC and TIC and contact the

335 translocation motor ${ }^{20,36}$. Highly structured sequence elements such as a helix at 
336 the Venus N-terminus ${ }^{37}$ can thus impede chloroplast targeting in the absence of a

337 sufficiently long presequence. HA-RAMPs with suitably unstructured C-termini,

338 such as SIM in this study, were thus most likely to generate chloroplast targeting

339 even in the absence of an added TP C-terminus. Similarly, higher chloroplast

340 targeting among cTP-C constructs may be due to mTP-C being more structured,

341 containing a long predicted amphipathic helix. Unstructured elements at $\mathrm{N}$ - and

342 C- termini may also contribute to chloroplast targeting by enabling interactions

343 with protein receptors associated with TOC. In basal algal lineages, a conserved F

344 at cTP N-termini appears to play a role in contacting TOC through an interaction

345 with cytoplasmic POTRA domains of TOC75 $38-40$, mimicking Omp85-interactions

346 in the bacterial cytoplasm mediated by C-terminal sequence motifs ${ }^{41}$, which may

347 make a TOC-interacting N-terminus an ancient feature of cTPs. In the green

348 lineage, TOC-interaction has instead been attributed to semi-conserved 'FGLK'

349 motifs $^{25,42-44}$, although the requirement for $F$ appears to be relaxed in

350 Chlamydomonas (Fig. 5G) ${ }^{45}$. The presence of GLK sites with high predicted

351 interactivity in cTP-N and cTP-C may explain the high chloroplast targeting

352 potential of constructs equipped with both elements. Thus the evolution of the

353 chloroplast import machinery and chloroplast-specific TPs likely went hand in

354 hand. To generate unstructured, protein interactive $\mathrm{N}$ - and C-termini, cTPs may

355 have evolved from HA-RAMPs that already contained unstructured sequence

356 elements (Fig. 8C), as seen for cp-set HA-RAMPs. Alternatively, cTPs may have

357 co-opted existing mTPs that already contained $\mathrm{R}$ and a cleavage site, yet were

358 still recognized by cyanobacterial HA-RAMP importers which were part of their

359 resistance mechanism to antimicrobial attack. In this case, the key innovation

360 that generated cTPs may simply have been the addition of an unstructured, 
361 possibly TOC-interacting N-terminus to mTPs (Fig. 8D), which we showed here

362 to be sufficient to retarget mt-set HA-RAMPs equipped with TP C-termini at least

363 partially to the chloroplast.

\section{The legacy of HA-RAMPs: Amphipathic helices and membrane interaction}

365 A direct interaction with the membrane bilayer, rather than protein receptors,

366 would provide a basic mechanism for a first step in organelle targeting and could

367 explain how TPs can be functionally specific whilst diverse in sequence. Core

368 components of the outer membrane translocons, such as the translocation pores

369 TOM40 and TOC75 are widely conserved, and both yeast TOM40 and plant

370 TOC75 retain the ability to interact with TPs in the absence of dedicated receptor

371 proteins ${ }^{46-48}$. In contrast to the core components, dedicated TP receptor proteins

372 are not conserved between the key eukaryotic lineages and should be regarded

373 as later addition to translocation machineries ${ }^{49,50}$. Where receptor interactions

374 are required for targeting, binding sites need to be restored when using

375 heterologously expressed proteins, e.g. a leading F needs to be added to plant

376 cTPs to generate cyanelle-targeting in glaucophytes ${ }^{51}$ (see previous paragraph).

377 Yet for the most part, heterologous expression of TPs across even large

378 phylogenetic distances generates reliable targeting in both plastids and

379 mitochondria ${ }^{52-54}$, such as ophistokont mTPs in trypanosomes and cyanelle cTPs

380 in plant chloroplasts. Most strikingly, chromatophore targeting peptides from

381 Paulinella chromatophora, an amoeba that has undergone a primary

382 endosymbiosis with an $\alpha$-cyanobacterium ca. 1 Mya, target tobacco

383 chloroplasts ${ }^{55}$. TP-enabled targeting must therefore use a universally shared

384 mechanism. In HA-RAMPs, amphipathic helices serve to insert into target 
membranes. Individual mTPs and cTPs have also been shown to interact with

membrane bilayers ${ }^{6,14}$. Our finding that targeting specificity is largely

387 determined by the sequence of the HA-RAMPs points to amphipathic helical

388 elements being directly involved in determining target specificity. While $\mathrm{N}$ - and

389 C-terminal elements aid specificity determination, particularly for chloroplast

390 import, their influence fails to explain why some HA-RAMPs target preferentially

391 the mitochondria or exclusively the chloroplast. In analogy to Rubisco small

392 subunit cTP interacting with chloroplast-mimetic membranes only in presence of

393 the chloroplast-specific galactolipid MGDG56, we suggest amphipathic helices

394 may interact specifically with the membranes of the targeted organelle ${ }^{3}$. Further

395 understanding of lipid interactions, the relationship between extant translocons

396 and bacterial resistance importers, as well as of the peptidases involved in the

397 degradation of TPs, should shed new light on the evolutionary process that

398 allowed the stable integration of proto-organelles through endosymbiosis.

399 Materials and Methods

400 Construct generation

401 Venus expression constructs were designed in SnapGene (v4.3.11) and 402 generated by integrating PCR-amplified (Q5 hot start high-fidelity, \#M0515, New

403 England Biolabs) DNA fragments into plasmid pODC53 ${ }^{57}$ upstream of Venus 404 using Gibson assembly (NEBuilder HiFi DNA assembly, \#E5520S, New England 405 Biolabs). Chlamydomonas TP sequences were amplified from genomic DNA 406 extracted from strain T222+ (CC-5101). Templates for codon optimized HA407 RAMP, RP and $\mathrm{R} \rightarrow \mathrm{K}$ modified $\mathrm{TP}$ sequences were obtained by gene synthesis 
408 (Eurofins Genomics). Correct assembly was verified by sequencing (Eurofins

409 Genomics). Linear transformation cassettes were generated through restriction

410 digest with EcoRV (New England Biolabs).

\section{Transformation and fluorescence screen conditions}

412 Constructs were transformed into wild-type strain T222+ (CC-5101) using the

413 protocol described previously ${ }^{58}$, except using $4 \mu \mathrm{l}$ of DNA at $2 \mu \mathrm{g} / \mu \mathrm{l}$.

414 Transformants selected for Paromomycin resistance were grown in $200 \mu \mathrm{l}$ TAP in

415 96-well plates under $50 \mu \mathrm{mol}$ photons $\mathrm{m}^{-2} \mathrm{~s}^{-2}$ for 3-5 days and then screened for

416 Venus expression in a fluorescence plate reader (CLARIOstar, BMG Labtech) as

417 described previously57.

418 Microscopy

419 Cells were grown in 96-well plates and epifluorescence microscopy was carried

420 out as described before ${ }^{5}$. Strains with suspected mitochondrial targeting were

421 subjected to $0.1 \mu \mathrm{M}$ MitoTracker Red CMXRos (ThermoFisher) in growth 422 medium for $30 \mathrm{~min}$ in the dark and washed with TAP prior to taking

423 epifluorescence images with the same settings as before ${ }^{5}$, using a white light LED

424 and a beam splitter at 535nm (423052-0104-000, Zeiss) in combination with a

425 custom-made filter set (559/34 BrightLine HC, Beamsplitter T 585 LP, 607/36

426 BrightLine HC; AHF Analysentechnik AG) for detecting the MitoTracker signal.

427 Images were adjusted in Fiji ${ }^{59}$ (ImgageJ version 2.0.0) as described before ${ }^{5}$, and

428 final figures assembled in PowerPoint (Microsoft PowerPoint for Mac 2011,

429 Version 14.6.3). 


\section{Western Blots}

431 Cells were grown up in $10 \mathrm{ml}$ TAP Erlenmeyer Flasks under $30 \mu \mathrm{mol}$ photons $\mathrm{m}^{-2}$ $432 \mathrm{~s}^{-1}$, shaken at 160RPM until late mid-log phase. $2 \mathrm{ml}$ Aliquots were harvested by

433 centrifugation at room temperature in a tabletop centrifuge, resuspended in $30 \mu \mathrm{l}$

434 storage buffer (1× Roche cOmplete ${ }^{\mathrm{TM}}$ Mini proteinase inhibitor cocktail, $10 \mathrm{mM}$

$\left.435 \mathrm{NaF}, 0.2 \mathrm{M} \mathrm{DTT}, 0.2 \mathrm{M} \mathrm{NaCO}_{3}\right)$ and kept at $-20^{\circ} \mathrm{C} .20 \mu \mathrm{l}$ boiling buffer $(1 \times$ Roche 436 cOmplete $^{\mathrm{TM}}$ Mini proteinase inhibitor cocktail, 10mM NaF, $50 \mathrm{~g} / \mathrm{L} \mathrm{SDS}, 200 \mathrm{~g} / \mathrm{L}$

437 sucrose) was added to thawed aliquots, which were then boiled for 50 seconds.

438 Cell debris was removed by centrifugation (tabletop centrifuge, maximum speed, 43915 minutes, $4^{\circ} \mathrm{C}$ ). Chlorophyll content of $5 \mu$ l supernatant in $1 \mathrm{ml}$ water was 440 estimated as $1 \mu \mathrm{g}$ chlorophyll $\mu \mathrm{l}^{-1}=0.11 \times\left(\mathrm{OD}_{680}-\mathrm{OD}_{770}\right)$, with Absorbances at $441680 \mathrm{~nm}\left(\mathrm{OD}_{680}\right)$ and $770 \mathrm{~nm}\left(\mathrm{OD}_{770}\right)$ recorded in a spectrophotometer. Samples 442 were diluted to load identical volumes, and a constant $10 \mu \mathrm{g}$ chlorophyll were 443 loaded onto large gels $(35 \times 27 \mathrm{~cm}$; resolving gel: $12 \%$ Acrylamide, $0.32 \%$ 444 Bisacrylamide, $0.25 \mu \mathrm{l} / \mathrm{ml}$ Tetramethylethylenediamine, $250 \mathrm{mg} / \mathrm{L}$ Ammonium 445 Persulfate, $375 \mathrm{mM}$ Tris/HCl pH 8.8; stacking gel: 5\% Acrylamide, $0.133 \%$ 446 Bisacrylamide, $0.666 \mu \mathrm{l} / \mathrm{ml}$ Tetramethylethylenediamine, $666 \mathrm{mg} / \mathrm{L}$ Ammonium 447 Persulfate, $125 \mathrm{mM}$ Tris/HCl $\mathrm{pH}$ 6.8) alongside a molecular size marker 448 (Precision Plus Dual Extra Prestained Protein Standards, BioRad), and gels run 449 overnight at room temperature at 18 A. Proteins in the size range $<25 \mathrm{kDA}$ to $450>75 \mathrm{kDa}$ were transferred onto $0.1 \mu \mathrm{m}$ nitrocellulose membranes for $1 \mathrm{~h}$ at $0.8 \mathrm{~A}$ $451 \mathrm{~cm}^{-2}$, using 5 layers of $3 \mathrm{~mm}$ filter paper (Whatman) soaked in transfer buffer 1 452 (40 mM Aminocaproic acid, 20\% Isopropanol, 25 mM Tris/HCl pH 9.4) between 453 at the cathode, followed by gel, membrane and 2 filter papers soaked in transfer 454 buffer 2 (20\% Isopropanol, $25 \mathrm{mM}$ Tris/HCl pH 10.4), and finally 3 filter papers 
455 soaked in transfer buffer 3 (20\% Isopropanol, $300 \mathrm{mM}$ Tris/HCl pH 10.4) at the

456 anode. Proteins were fixed and visualized by Ponceau Red staining, incubated for

$4571 \mathrm{~h}$ at room temperature in block (30 g/L skimmed milk powder, $0.1 \%$ tween-20,

458 1x PBS: $140 \mathrm{mM} \mathrm{NaCl}, 30 \mathrm{mM} \mathrm{KCl}, 100 \mathrm{mM} \mathrm{Na} 2 \mathrm{HPO}_{4}, 15 \mathrm{mM} \mathrm{KH}_{2} \mathrm{PO}_{4}$ ) and

459 immunolabelled overnight at $4^{\circ} \mathrm{C}$ using an $\alpha$-FLAG $1^{\circ}$ antibody (Sigma Aldrich

460 F1804, diluted 1:10 000 in block). Membranes were thoroughly washed $(0.1 \%$

461 tween-20, 1x PBS), treated for $1 \mathrm{~h}$ at room temperature with horseradish

462 peroxidase-conjugated $\alpha$-mouse $2^{\circ}$ antibody (diluted 1:10 000 in block), washed

463 again and revealed using ECL recorded on a ChemiDoc (Biorad). Blots were

464 analyzed and converted into TIFF format using the Image Lab software (Version

465 6.0.0 build 26, BioRad), final figures were assembled in PowerPoint (Microsoft).

466 Antimicrobial activity assay

467 Minimum inhibitory concentration assays were performed as described before ${ }^{5}$.

468 Sequence Data Set

469 TPs with experimentally-confirmed cleavage sites were recovered from 470 proteomic studies: Chlamydomonas reinhardtii cTP (Terashima 2011), mTP 471 (Tardif 2012); Arabidopsis thaliana cTP (Ge 2008, Rowland 2015), mTP (Huang 472 2009). For each peptide, we recovered the full-length protein sequence from

473 NCBI and UNIPROT. Cytoplasmic control sequences were generated by taking N-

474 terminal sequence stretches of random length, matching the distribution of 475 peptide lengths observed in our Chlamydomonas TP dataset, from a random 476 subset of Chlamydomonas proteins with a validated cytoplasmic location in 477 UNIPROT. For calculating amino acid frequencies, the same HA-RAMP sequences 478 were used as before ${ }^{5}$. 


\section{Amphipathic helix prediction}

480 Amphipatic $\alpha$-helices are predicted as described previously, following the 481 principle of the Heliquest algorithm ${ }^{60}$. Briefly, the approach aims to establish the

482 longest sequence stretch that contains identifiable hydrophilic and hydrophobic

483 faces. The algorithm is iterated such that multiple non-overlapping helices can be 484 found within a given peptide (Fig. 1). Consequently, helix fractions are calculated 485 as number of residues within all predicted helices divided by the total number of 486 residues in the peptide; for evaluating the number of upstream residues, only the 487 longest helix was considered (Fig. 7, Sup. Fig. 23).

488 ACC terms

489 In order to evaluate the physicochemical properties of our peptides we used the 490 approach we previously described ${ }^{5}$. Briefly, each amino acid is described in 491 terms of 3 'Z-scale' values ${ }^{61}$ that can be interpreted as representing a residue's 492 hydrophobic, steric and electronic properties. Auto-cross-covariances (ACC) 493 between nearby residues are calculated up to a distance of four amino acids, 494 generating a quantitative representation of a given peptide in terms of 36 ACC 495 terms.

\section{Visualization}

497 We performed a principal component analysis (PCA) to visualize the 498 relationships between peptides as described by their 36 ACC terms (Sup. Fig. 20) 499 or by their 5 salient TP properties (Sup. Fig. 23) using python package sklearn $500 \quad \mathrm{v} 0.22 .1^{62}$ 
502 TP N-termini, defined as the N-terminal 15 amino acids, were analysed (Sup. Fig.

503 21) as follows: Charge profiles were generated as described in the literature ${ }^{25}$.

504 Hydrophobicity of TP N-termini was estimated using the Heliquest standalone

505 application ${ }^{60}$. To evaluate disorder, we used IUpred2A, a software that calculates

506 the probability for each residue of being in a disordered region ${ }^{63}$. We used the

507 default 'Long' setting, which has been reported to be more accurate than the

508 alternative 'Short' setting ${ }^{64}$. The disorder of a given sequence was taken as the

509 mean of the probability values for each residue (average residue disorder

510 probability).

511 Statistical prediction

512 To evaluate the predictive power of ACC terms ${ }^{5}$ obtained for 15 residue-long TP

513 N-termini with regards to localization (Sup. Fig. 21) we used a binomial logistic

514 regression classifier. We performed one hundred 5-fold cross validation runs. In

515 each set of one hundred runs, we randomly selected sequences such that the

516 same number of mTPs and cTPs were used. For $C$. reinhardtii we used 33 mTP

517 and 33 cTP and for $A$. thaliana we use 29 mTP and 29 cTP sequences. We used an

518 elastic net penalty in a saga solver with an 11-ratio (a-parameter) of 0 , which is

519 equivalent to using only ridge penalty, where all features take part in the model,

520 and a $C$ parameter $(1 / \lambda)$ of 0.1 . The a and $1 / \lambda$ parameters were optimised with

52110 -fold cross validation. Firstly, when $1 / \lambda=1$, the best accuracy (0.82) was

522 obtained with a between 0 and 0.09 . Secondly, with $\mathrm{a}=0$, the best accuracy

523 (0.82) was obtained with $1 / \lambda=0.1$. Custom scripts were written in python

524 (v3.7.6). 
526 Values for the Boman index, a quantitative proxy for whether a peptide is more

527 likely to interact with proteins (high values) or lipids (low values), were

528 calculated as described in the literature ${ }^{65}$. Anchor2 interactivity values, a second

529 proxy for protein interaction potential developed for disordered sequences ${ }^{66}$,

530 were calculated using the IUPred2A standalone application and webserver 63,67 .

531 Interaction sites for Hsp70 were predicted as described by Ivey and coworkers ${ }^{23}$

532 based on experimental affinity values for individual amino acids. Putatively TOC-

533 interacting 'FGLK' sites were established by searching for presence of $\mathrm{F}$ and [P or

$534 \mathrm{G}$ ] and [K or R] and [A or L or V], and absence of [D and E], within each 8-residue

535 window of a sequence, corresponding to rule 22 by Chotewutmontri and co-

536 workers ${ }^{25}$ that was recommended by the authors in personal communication.

537 'FGLK-1' sites were established the same way, but requiring the presence of only

538 three of the four positive determinants. Custom scripts were implemented in $\mathrm{R}^{68}$

539 (v3.6.1) using RStudio ${ }^{69}$ (v1.2.1335).

\section{$540 \quad$ Statistical analysis}

541 Chlamydomonas TP distributions (Fig. 6, Sup.Fig. 24) were compared using two-

542 sided t-tests ( $n=34$ for mTPs and $n=85$ for cTPs), and associated mt-set and cp-

543 set HA-RAMPs were compared using one-sided t-tests based on the trends set by

544 TPs ( $n=3$ for mt-set and $n=5$ for cp-set HA-RAMPs) in $\mathrm{R}^{68}$ using RStudio ${ }^{69}$.

545 Multiple Kruskal statistical tests are performed (same Chlamydomonas mTPs

546 and cTPs as above, plus $n=382$ HA-RAMPs) to evaluate the distribution of amino

547 acids (Fig. 4A, Sup. Fig. 19) in the different groups, followed by Dunn post-hoc

548 tests (scipy v1.4.1). 


\section{Acknowledgments}

550 Thank you to Tiffina Benhamou and Gabriel Chemin, who worked on aspects of

551 this study with ODC during their summer internships. We also thank Prakitchai

552 Chotwutmontri and Barry Bruce for their help in correctly replicating their

553 Hsp70-binding and FGLK-motif finding algorithms.

554 Financial support by annual funding from the Centre National de la Recherche

555 Scientifique and Sorbonne University to UMR 7141, by the ChloroMitoRAMP

556 ANR grant (ANR-19-CE13-0009) and by LabEx Dynamo (ANR-LABX-011), as

557 well as by the Rothschild Foundation, is gratefully acknowledged.

\section{Competing interest statement}

559 The authors declare no competing interests. The funders had no role in the

560 design of the study; in the collection, analyses, or interpretation of data; in the

561 writing of the manuscript, or in the decision to publish the results.

\section{Data availability statement}

563 The authors confirm that the data supporting the findings of this study are

564 available within the article and its supplementary materials.

\section{Code availability statement}

566 Custom code generated in the course of this project will be made available

567 without restrictions upon request to the authors. 


\section{Materials availability statement}

569 All constructs and an informative subset of expressing strains will be made

570 available through the Chlamydomonas resource centre.

\section{Author contributions}

572 FAW, IL and YC conceptualized the project and acquired funds. ODC devised,

573 conducted and visualized the wet-lab experimental part of the investigation, and

574 was chiefly responsible for assigning subcellular localization interpretations

575 based on microscopic evidence. CG carried out the dry-lab/bioinformatic part of

576 the investigation and associated visualization under the supervision of IL,

577 writing custom software to do so (ODC contributed to the analysis of FGLK and

578 Hsp70-binding sites). ODC wrote the original draft of the manuscript. FAW, IL,

579 ODC and YC reviewed and edited the manuscript. All authors approved the final

580 manuscript.

\section{References}

582 1. Wollman, F.-A. An antimicrobial origin of transit peptides accounts for

583 early endosymbiotic events. Traffic 17, 1322-1328 (2016).

2. Caspari, O. D. \& Lafontaine, I. The role of antimicrobial peptides in the evolution of endosymbiotic protein import. PLoS Pathog. 17, e1009466 586 (2021).

587 3. Lazzaro, B. P., Zasloff, M. \& Rolff, J. Antimicrobial peptides: Application $588 \quad$ informed by evolution. Science 368, (2020).

589 4. Joo, H. S., Fu, C. I. \& Otto, M. Bacterial strategies of resistance to $590 \quad$ antimicrobial peptides. Philos. Trans. R. Soc. B Biol. Sci. 371, (2016).

591 5. Garrido, C., Caspari, O. D., Choquet, Y., Wollman, F. A. \& Lafontaine, I. 592 Evidence Supporting an Antimicrobial Origin of Targeting Peptides to Endosymbiotic Organelles. Cells 9, 1795 (2020). 
594

595

596

597

598

599

600

601

602

603

604

605

606

607

608

609

610

611

612

613

614

615

616

617

618

619

620

621

622

623

624

625

626

627

628

629

630

6. von Heijne, G., Steppuhn, J. \& Herrmann, R. G. Domain structure of mitochondrial and chloroplastic targeting peptides. Eur. J. Biochem. 180, 535-545 (1989).

7. von Heijne, G. Mitochondrial targeting sequences may form amphiphilic helices. EMBO J. 5, 1335-1342 (1986).

8. von Heijne, G. \& Nishikawa, K. Chloroplast transit peptides. The perfect random coil? FEBS Lett. 278, 1-3 (1991).

9. Franzén, L.-G. G., Rochaix, J.-D. D. \& von Heijne, G. Chloroplast transit peptides from the green alga Chlamydomonas reinhardtii share features with both mitochondrial and higher plant chloroplast presequences. FEBS Lett. 260, 169-172 (1990).

10. Lancelin, J. M. et al. NMR structures of a mitochondrial transit peptide from the green alga Chlamydomonas reinhardtii. FEBS Lett. 391, 203-208 (1996).

11. Krimm, I., Gans, P., Hernandez, J. F., Arlaud, G. J. \& Lancelin, J. M. A coil-helix instead of a helix-coil motif can be induced in a chloroplast transit peptide from Chlamydomonas reinhardtii. Eur. J. Biochem. 265, 171-180 (1999).

12. Wienk, H. L. J., Wechselberger, R. W., Czisch, M. \& Kruijff, B. De. Structure , Dynamics, and Insertion of a Chloroplast Targeting Peptide in Mixed. 8219-8227 (2000).

13. Bruce, B. D. The paradox of plastid transit peptides: Conservation of function despite divergence in primary structure. Biochim. Biophys. Acta Mol. Cell Res. 1541, 2-21 (2001).

14. Bruce, B. D. The role of lipids in plastid protein transport. Plant Mol. Biol. 38, 223-46 (1998).

15. Teixeira, P. F. \& Glaser, E. Processing peptidases in mitochondria and chloroplasts. Biochim. Biophys. Acta - Mol. Cell Res. 1833, 360-370 (2013).

16. Köhler, D. et al. Identification of protein N-termini in Cyanophora paradoxa cyanelles: transit peptide composition and sequence determinants for precursor maturation. Front. Plant Sci. 6, 1-11 (2015).

17. Tardif, M. et al. Predalgo: A new subcellular localization prediction tool dedicated to green algae. Mol. Biol. Evol. 29, 3625-3639 (2012).

18. Bionda, T. et al. Chloroplast import signals: The length requirement for translocation in vitro and in vivo. J. Mol. Biol. 402, 510-523 (2010).

19. Caspari, O. D. Chloroplast transit peptides often require downstream unstructured sequence in Chlamydomonas reinhardtii. bioRxiv (2021). doi:https://www.biorxiv.org/content/10.1101/2021.11.26.470094 
20. Chotewutmontri, P., Holbrook, K. \& Bruce, B. D. Plastid Protein Targeting: Preprotein Recognition and Translocation. Int. Rev. Cell Mol. Biol. 330, 227-294 (2017).

21. Lee, D. W. et al. Molecular Mechanism of the Specificity of Protein Import into Chloroplasts and Mitochondria in Plant Cells. Mol. Plant (2019). doi:10.1016/j.molp.2019.03.003

22. Bhushan, S., Kuhn, C., Berglund, A., Roth, C. \& Glaser, E. The role of the Nterminal domain of chloroplast targeting peptides in organellar protein import and miss-sorting. FEBS 580, 3966-3972 (2006).

23. Ivey, R. a, Subramanian, C. \& Bruce, B. D. Identification of a Hsp70 recognition domain within the rubisco small subunit transit peptide. Plant Physiol. 122, 1289-1299 (2000).

24. Ivey, R. a \& Bruce, B. D. In vivo and in vitro interaction of DnaK and a chloroplast transit peptide. Cell Stress Chaperones 5, 62-71 (2000).

25. Chotewutmontri, P., Reddick, L. E., McWilliams, D. R., Campbell, I. M. \& Bruce, B. D. Differential Transit Peptide Recognition during Preprotein Binding and Translocation into Flowering Plant Plastids. Plant Cell Online 24, 3040-3059 (2012).

26. Chotewutmontri, P. \& Bruce, B. D. Non-native, N-terminal Hsp70 molecular motor recognition elements in transit peptides support plastid protein translocation. J. Biol. Chem. 290, 7602-7621 (2015).

27. Rothbard, J. B., Jessop, T. C., Lewis, R. S., Murray, B. A. \& Wender, P. A. Role of membrane potential and hydrogen bonding in the mechanism of translocation of guanidinium-rich peptides into cells. J. Am. Chem. Soc. 126, 9506-9507 (2004).

28. Mitchell, D. J., Kim, D. T., Steinman, L., Fathman, C. G. \& Rothbard, J. B. Polyarginine enters cells more efficiently than other polycationic homopolymers. J. Pept. Res. 56, 318-25 (2000).

29. Li, L., Vorobyov, I. \& Allen, T. W. The different interactions of lysine and arginine side chains with lipid membranes. J. Phys. Chem. B 117, 1190611920 (2013).

30. Li, J. et al. Membrane Active Antimicrobial Peptides: Translating Mechanistic Insights to Design. Front. Neurosci. 11, 1-18 (2017).

31. Mattiroli, F. \& Sixma, T. K. Lysine-targeting specificity in ubiquitin and ubiquitin-like modification pathways. Nat. Struct. Mol. Biol. 21, 308-316 (2014).

32. Kmiec, B., Teixeira, P. F. \& Glaser, E. Shredding the signal: targeting peptide degradation in mitochondria and chloroplasts. Trends Plant Sci. 19, 771-8 (2014). 
33. Lee, D. W. et al. Cross-Species Functional Conservation and Possible Origin of the N-Terminal Specificity Domain of Mitochondrial Presequences. Front. Plant Sci. 11, 1-7 (2020).

34. Garg, S. G. \& Gould, S. B. The Role of Charge in Protein Targeting Evolution. Trends Cell Biol. 26, 894-905 (2016).

35. Martin, J., Mahlke, K. \& Pfanner, N. Role of an energized inner membrane in mitochondrial protein import: $\Delta \Psi$ drives the movement of presequences. J. Biol. Chem. 266, 18051-18057 (1991).

36. Nakai, M. New Perspectives on Chloroplast Protein Import. Plant Cell Physiol. 59, 1111-1119 (2018).

37. Rekas, A., Alattia, J. R., Nagai, T., Miyawaki, A. \& Ikura, M. Crystal structure of venus, a yellow fluorescent protein with improved maturation and reduced environmental sensitivity. J. Biol. Chem. 277, 50573-50578 (2002).

38. Knopp, M., Garg, S. G., Handrich, M. \& Gould, S. B. Major Changes in Plastid Protein Import and the Origin of the Chloroplastida. iScience 23, 100896 (2020).

39. Sommer, M. S. et al. Chloroplast Omp85 proteins change orientation during evolution. Proc. Natl. Acad. Sci. U. S. A. 108, 13841-13846 (2011).

40. Wunder, T., Martin, R., Löffelhardt, W., Schleiff, E. \& Steiner, J. M. The invariant phenylalanine of precursor proteins discloses the importance of Omp85 for protein translocation into cyanelles. BMC Evol. Biol. 7, 236 (2007).

41. Robert, V. et al. Assembly factor Omp85 recognizes its outer membrane protein substrates by a species-specific C-terminal motif. PLoS Biol. 4, 1984-1995 (2006).

42. Holbrook, K. et al. Functional Analysis of Semi-conserved Transit Peptide Motifs and Mechanistic Implications in Precursor Targeting and Recognition. Mol. Plant 9, 1286-1301 (2016).

43. Pilon, M. et al. Functional domains of the ferredoxin transit sequence involved in chloroplast import. Journal of Biological Chemistry 270, 38823893 (1995).

44. Lee, D. W., Lee, S., Oh, Y. J. \& Hwang, I. Multiple sequence motifs in the rubisco small subunit transit peptide independently contribute to Toc159dependent import of proteins into chloroplasts. Plant Physiol. 151, 129141 (2009).

45. Razzak, A., Lee, D. W., Yoo, Y. \& Hwang, I. Evolution of rubisco complex small subunit transit peptides from algae to plants. Sci. Rep. 7, 9279 (2017). 
46. Hill, K. et al. Tom 40 forms the hydrophilic channel of the mitochondrial import pore for preproteins. Nature 395, 516-521 (1998).

47. Hinnah, S. C., Hill, K., Wagner, R., Schlicher, T. \& Soll, J. Reconstitution of a chloroplast protein import channel. EMBO J. 16, 7351-7360 (1997).

48. Hinnah, S. C., Wagner, R., Sveshnikova, N., Harrer, R. \& Soll, J. The chloroplast protein import channel Toc75: pore properties and interaction with transit peptides. Biophys. J. 83, 899-911 (2002).

49. Duncan, O., Murcha, M. W. \& Whelan, J. Unique components of the plant mitochondrial protein import apparatus. Biochim. Biophys. Acta - Mol. Cell Res. 1833, 304-313 (2013).

50. Schneider, A. Evolution of mitochondrial protein import - Lessons from trypanosomes. Biol. Chem. 401, 663-676 (2020).

51. Steiner, J. M. \& Löffelhardt, W. Protein translocation into and within cyanelles. Mol. Membr. Biol. 22, 123-132 (2005).

52. Atkinson, N. et al. Introducing an algal carbon-concentrating mechanism into higher plants: Location and incorporation of key components. Plant Biotechnol. J. 14, 1302-1315 (2016).

53. Steiner, J. M., Yusa, F., Pompe, J. A. \& Löffelhardt, W. Homologous protein import machineries in chloroplasts and cyanelles. Plant J. 44, 646-652 (2005).

54. Mani, J., Meisinger, C. \& Schneider, A. Peeping at TOMs - Diverse entry gates to mitochondria provide insights into the evolution of eukaryotes. Mol. Biol. Evol. 33, 337-351 (2016).

55. Singer, A. et al. Massive Protein Import into the Early-Evolutionary-Stage Photosynthetic Organelle of the Amoeba Paulinella chromatophora. Curr. Biol. 27, 2763-2773 (2017).

56. Pinnaduwage, P. \& Bruce, B. D. In vitro interaction between a chloroplast transit peptide and chloroplast outer envelope lipids is sequence-specific and lipid class-dependent. J. Biol. Chem. 271, 32907-32915 (1996).

57. Caspari, O. D. Introduction of a leaky stop codon as molecular tool in Chlamydomonas reinhardtii. PLoS One 15, e0237405 (2020).

58. Onishi, M. \& Pringle, J. R. Robust Transgene Expression from Bicistronic mRNA in the Green Alga Chlamydomonas reinhardtii. G3 (Bethesda). 6, 4115-4125 (2016).

59. Schindelin, J. et al. Fiji: an open-source platform for biological-image analysis. Nat. Methods 9, 676-682 (2012).

60. Gautier, R., Douguet, D., Antonny, B. \& Drin, G. HELIQUEST: a web server to 
screen sequences with specific alpha-helical properties. Bioinformatics 24, 2101-2102 (2008).

748

749

750

751

752

753

754

755

756

757

758

759

760

761

762

763

764

765

766

767

768

61. Hellberg, S., Sjostrom, M., Skagerberg, B. \& Wold, S. Peptide Quantitative Structure-Activity Relationships, a Multivariate Approach. J. Med. Chem. 30, 1126-1135 (1987).

62. Pedregosa, F. et al. Scikit-learn: Machine Learning in Python. J. Mach. Learn. Res. 12, 2825-2830 (2011).

63. Erdős, G. \& Dosztányi, Z. Analyzing Protein Disorder with IUPred2A. Curr. Protoc. Bioinforma. 70, 1-15 (2020).

64. Nielsen, J. T. \& Mulder, F. A. A. Quality and bias of protein disorder predictors. Sci. Rep. 9, 1-11 (2019).

65. Boman, H. G. Antibacterial peptides: basic facts and emerging concepts. J. Intern. Med. 254, 197-215 (2003).

66. Mészáros, B., Simon, I. \& Dosztányi, Z. Prediction of Protein Binding Regions in Disordered Proteins. PLoS One 5, e1000376 (2009).

67. Mészáros, B., Erdös, G. \& Dosztányi, Z. IUPred2A: Context-dependent prediction of protein disorder as a function of redox state and protein binding. Nucleic Acids Res. 46, W329-W337 (2018).

68. R Core Team. R: A Language and Environment for Statistical Computing. (2019).

69. RStudio Team. RStudio: Integrated Development for R. (2020).

70. Meyer, M. et al. Assembly of the algal CO2-fixing organelle, the pyrenoid, is guided by a Rubisco-binding motif. Sci. Adv. 6, eabd2408 (2020). 


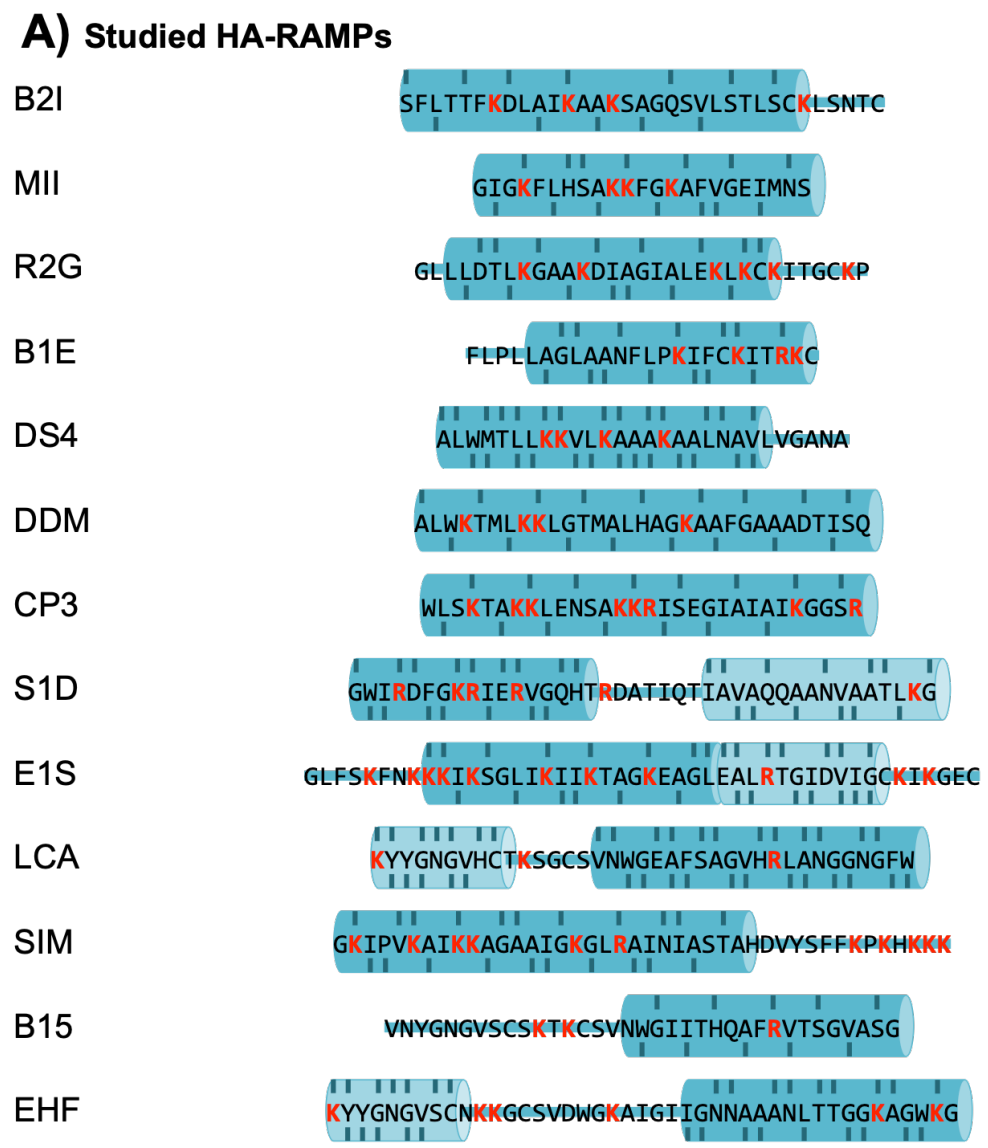

\section{B) TP controls}

RBCA-cTP

C) Negative control peptides

$\begin{array}{ll}\text { RP1 } & \text { NIVVYWNFTLWHMDINARNAGCDGEGS } \\ \text { RP2 } & \text { DEVNNDNCRIKFIKGDISSESDIKMNINY }\end{array}$

Figure 1. Peptide sequences under study. Amino acid sequences are shown

773 using the one-letter code. Positively charged residues are highlighted in red. The

774 fraction of the sequence predicted to fold into amphipathic helices (see Methods)

775 is provided by a pie-chart to the right of the sequence; for TPs in (B) this was 
776 calculated up to the cleavage site indicated by a downward arrow. Predicted

777 amphipathic helices are highlighted using a cylinder cartoon, with residues

778 contributing to the hydrophilic/hydrophobic face indicated on the top/bottom.

779 No helix could be predicted within RBCA-cTP, and thus the indicated helix is

780 taken from a published NMR-structure obtained in membrane-mimetic

781 conditions ${ }^{11}$. Note that the two helices of E1S are at an angle to each other and

782 therefore cannot form a single continuous amphipathic helix. Abbreviations: A)

783 B2I: Brevinin 2ISb, R2G: Ranatuerin 2G, MII: Magainin 2, B1E: Brevinin 1E, DS4:

784 Dermaseptin S4, DDM: Dermadistinctin M, CP3: Cecropin P3, S1D: Sarcotoxin 1D,

785 E1S: Esculentin 1S, LCA: Leucocin A, SIM: SI Moricin, B15: Bacillocin 1580, EHF:

786 Enterocin HF; B) TP - targeting peptide, cTP: chloroplast TP, mTP: mitochondrial

787 TP, RBCA: Rubisco activase, CAG2: $\gamma$-carbonic anhydrase, cTP-N: cTP N-terminal

788 element, CH: cTP helix, MH: mTP helix, cTP-C: cTP C-terminal element, mTP-C:

789 mTP C-terminal element; C) RP: random peptide. 


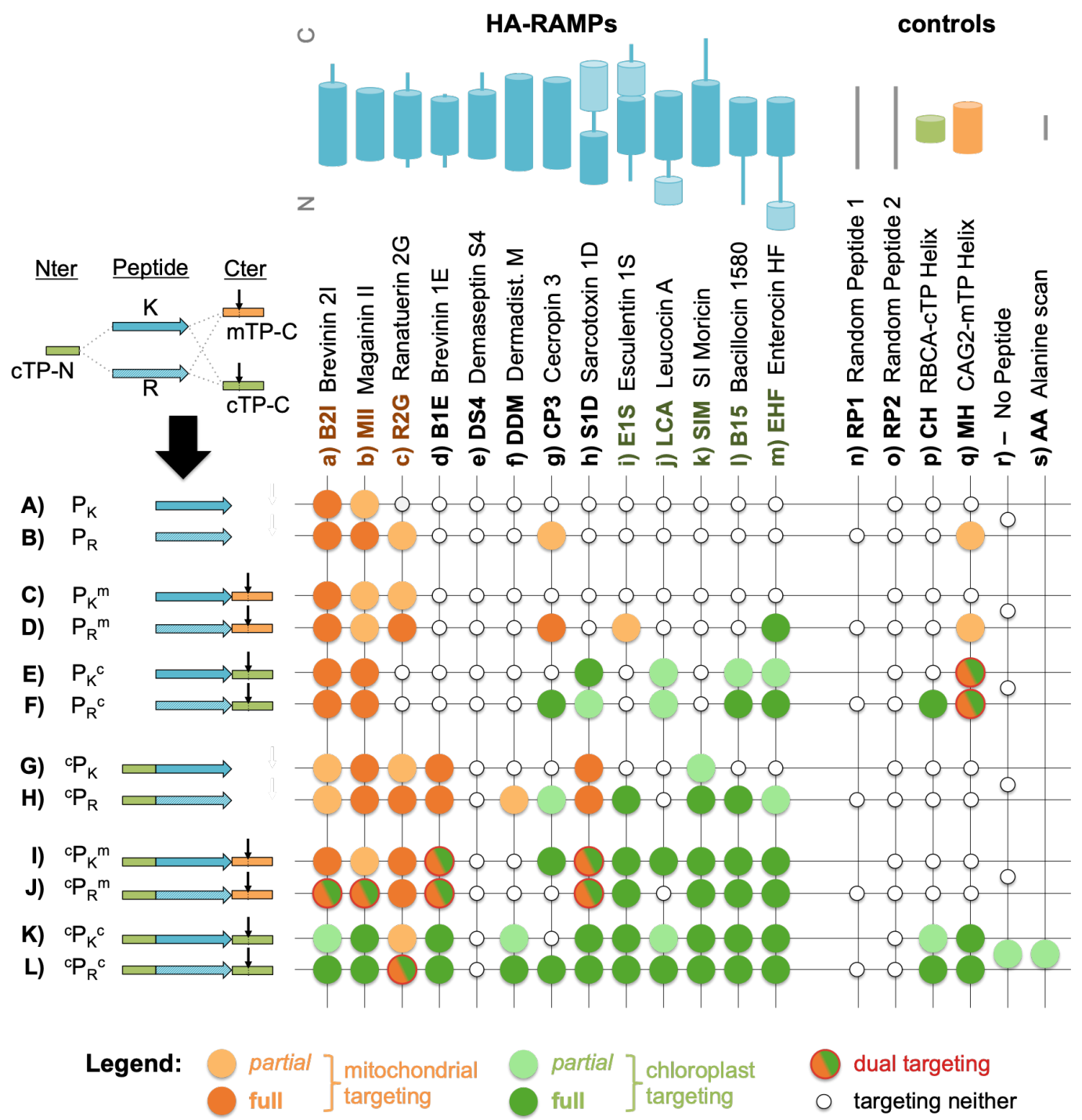

791 Figure 2. TP modifications enable HA-RAMP targeting. Chimeric constructs

792 were generated by combining TP elements with HA-RAMPs (see Fig. 1 for

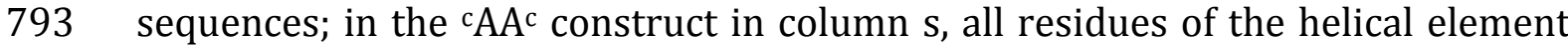
794 "CH" within RBCA-cTP are replaced by Alanines). The overview graph shows in 795 each column (a-s) one of the peptides with a cartoon indicating the position of 796 the predicted amphipathic helices within the sequence; and in each row (A-L) a 797 combination of peptide, K/R modification and TP-element indicated by a cartoon 798 and the following shorthand: $\mathrm{P}=$ peptide, $\mathrm{K}=$ contains mostly Lysines, $\mathrm{R}=$ 799 contains mostly Arginines, ${ }^{m}=\mathrm{mTP}$ element, ${ }^{c}=\mathrm{cTP}$ element $\left({ }^{\mathrm{c} P}=\mathrm{cTP}-\mathrm{N}, \mathrm{P}^{\mathrm{c}}=\right.$ 800 cTP-C). In each case, an overview of observed targeting is provided by a colour 
802 Fig. 1-18. Note that the present results for $B 2 \mathrm{I}_{K}{ }^{c}, \mathrm{MII}_{\mathrm{K}}^{\mathrm{c}}, \mathrm{S} 1 \mathrm{D}_{\mathrm{K}}^{\mathrm{c}}, \mathrm{B} 15_{\mathrm{K}}^{\mathrm{c}}$ and $\mathrm{EHF}_{\mathrm{K}}^{\mathrm{c}}$

803 (row E, columns a,b,h,l,m) confirmed our previous report on these strains ${ }^{5}$.

804

\section{MitoTracker Venus Chlorophyll Merge}
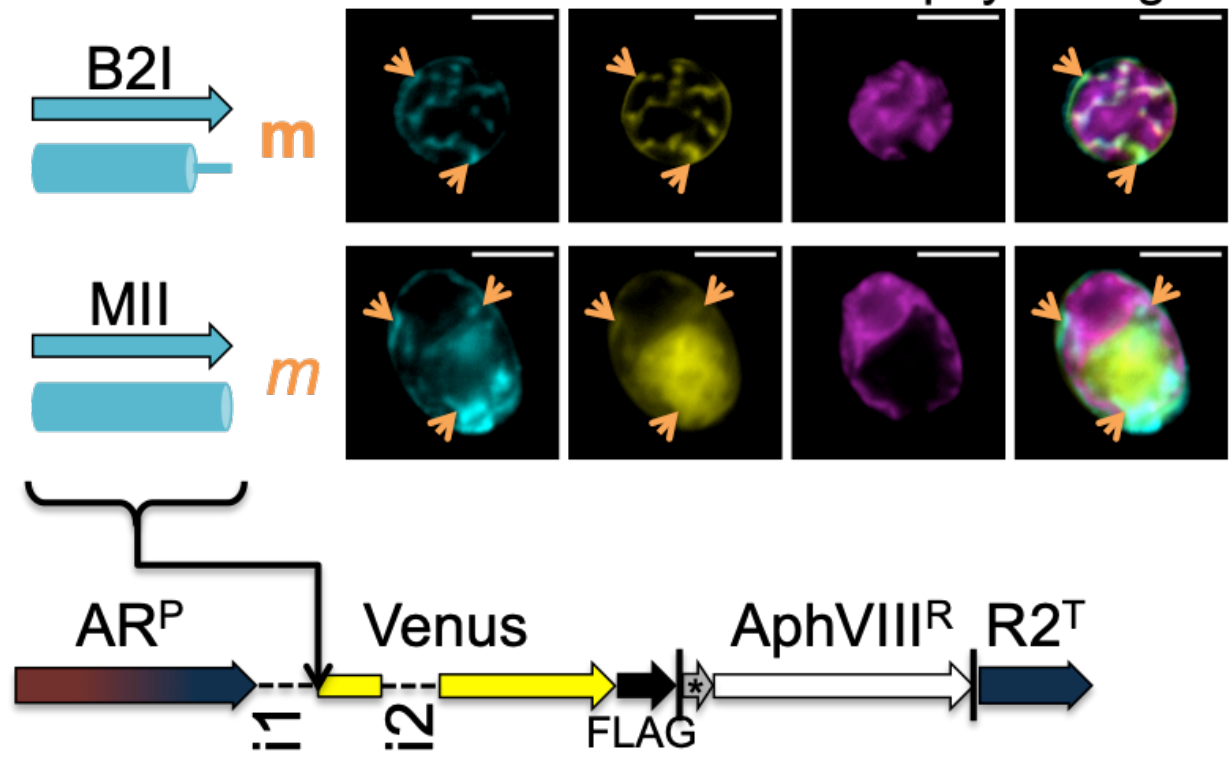

Figure 3. Unmodified HA-RAMPs can target the mitochondria. Indicated

peptides were inserted upstream of a Venus fluorescent protein reporter in a bicistronic expression system for Chlamydomonas ${ }^{57}$. $\mathrm{AR}^{\mathrm{P}}$ : hybrid HSP70a-RBCS2 promoter, i1: RBCS2 intron 1, i2: RBCS2 intron 2, FLAG: FLAG-tag, |: stop codon, *: bicistronic bridge sequence tagcat, AphVIII ${ }^{R}$ : Paromomycin resistance gene, $\mathrm{R}^{\mathrm{T}}$ : RBCS2 terminator. Epifluorescence microscopy images of selected examples are shown. False-coloured yellow fluorescence from the Venus channel reports on the subcellular localization of the fluorescent reporter. MitoTracker fluorescence, false-coloured in cyan, indicates the position of mitochondria (although parts of the cell exterior are sometimes also stained), with salient features highlighted with orange arrows to indicate co-localisation with the Venus channel. Chlorophyll autofluorescence, shown in magenta, indicates the location of the chloroplast. Scale bars are $5 \mu \mathrm{m}$. Refer to Fig. 1 for sequences and 
818 Sup. Fig. 1 and 2 for biological replicates. Where a construct was interpreted as

819 generating reporter localization in mitochondria or chloroplast, this is indicated

820 by an orange ' $m$ ' or a green ' $c$ ' respectively, in bold for full targeting or in italics

821 for partial targeting.

A) Basic residues differ

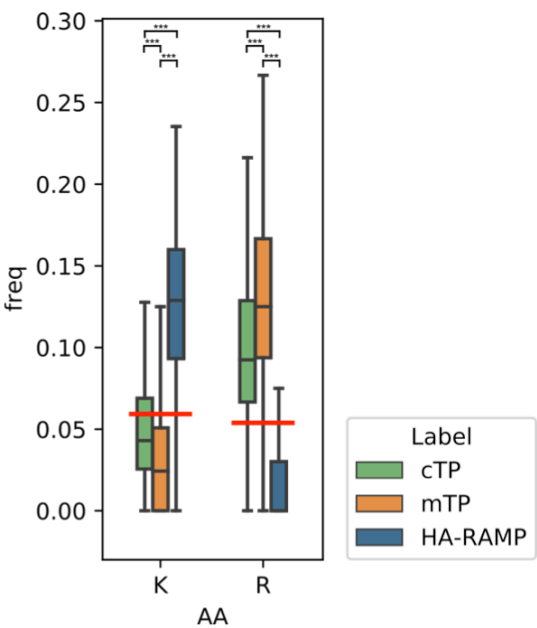

B) $K \rightarrow R$ reduces AMP activity

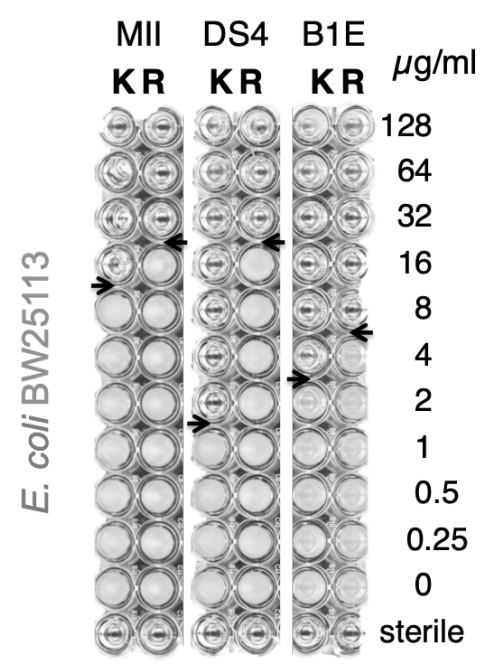

C) $R \rightarrow K$ reduces TP targeting

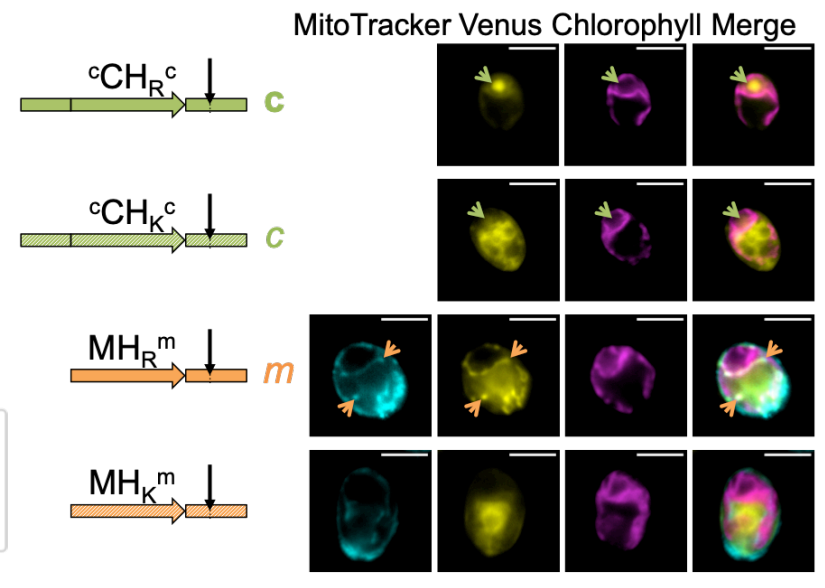

D) $K \rightarrow R$ increases HA-RAMP targeting

MitoTracker Venus Chlorophyll Merge
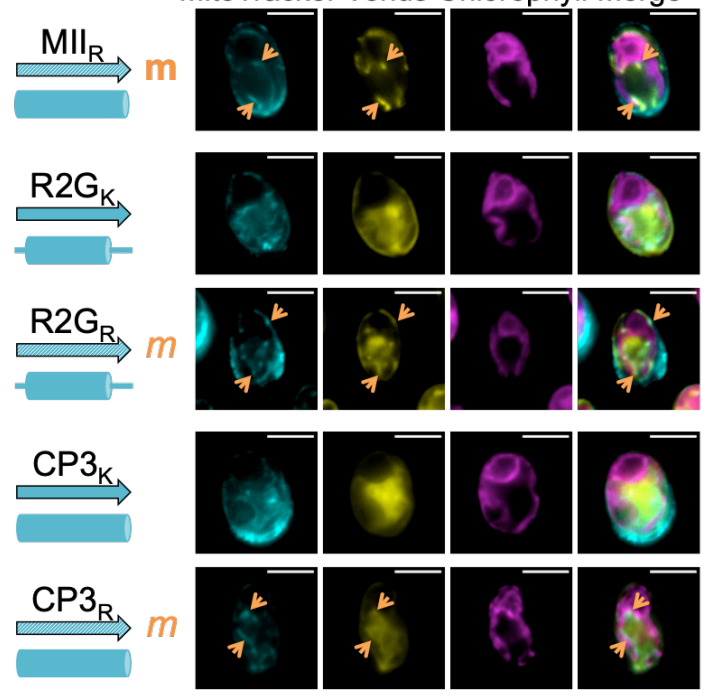

822

823 Figure 4. $\mathbf{K}$ is for killing, $\mathbf{R}$ is for targeting. A) Lysine (K) and Arginine (R)

824 frequencies for Chlamydomonas TPs and HA-RAMPs are shown as boxplots

825 (center line: median; box limits: upper and lower quartiles; whiskers: $\min / \max$ 826 values within $1.5 \mathrm{x}$ interquartile range). To give a baseline for comparison, their 827 average across the Uniprot database is given as red horizontal line. Statistically 
828 significant differences are indicated with stars: (Multiple Kruskal-Wallis tests

829 followed by Dunn post-hoc tests, $\left.{ }^{* * *} \mathrm{p}<0.0001\right)$. See Sup. Fig. 19 for all amino

830 acids. B) Grayscale photos of antimicrobial activity growth assays in the

831 presence of dilutions of three selected antimicrobial peptides against E. coli

832 strain BW25113 show reduced activity when natively K-rich sequences (K, left

833 hand columns) are altered by replacing all Ks by Rs ( $\mathrm{R}$, right hand columns). C

834 and D) Epifluorescence images of selected chimeric TP control (C) or HA-RAMP

835 (D) constructs are shown as in Fig. 3. MH: mTP helical fragment, CH: cTP helical

836 fragment. Orange arrows highlight co-localisation of Venus signal with the

837 MitoTracker dye, demonstrating mitochondrial localisation; green arrows

838 highlight the presence of Venus signal in the pyrenoid, demonstrating

839 chloroplast localization. Where a construct was interpreted as generating

840 reporter localization in mitochondria or chloroplast, this is indicated by an

841 orange ' $m$ ' or a green 'c' respectively. 
A) mTP-C: mitochondrial targeting MitoTracker Venus Chlorophyll Merge

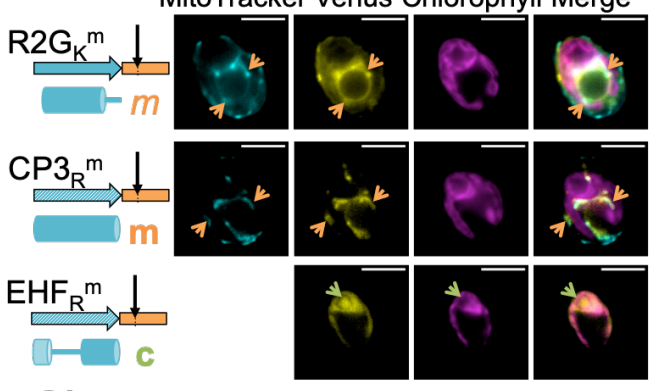

C) cTP-C is important for chloroplast
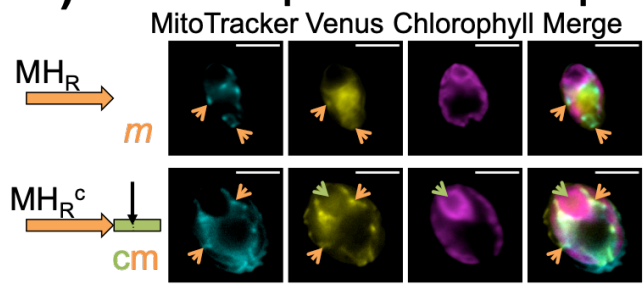

D) cTP-N: chloroplast targeting
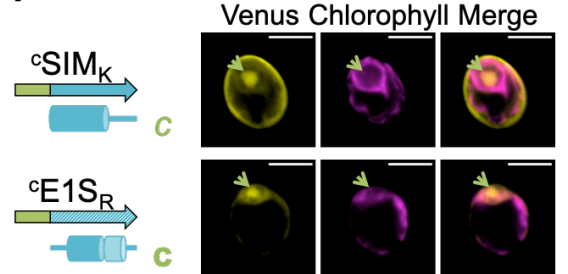

F) cTP-N interacts with C-ter to determ
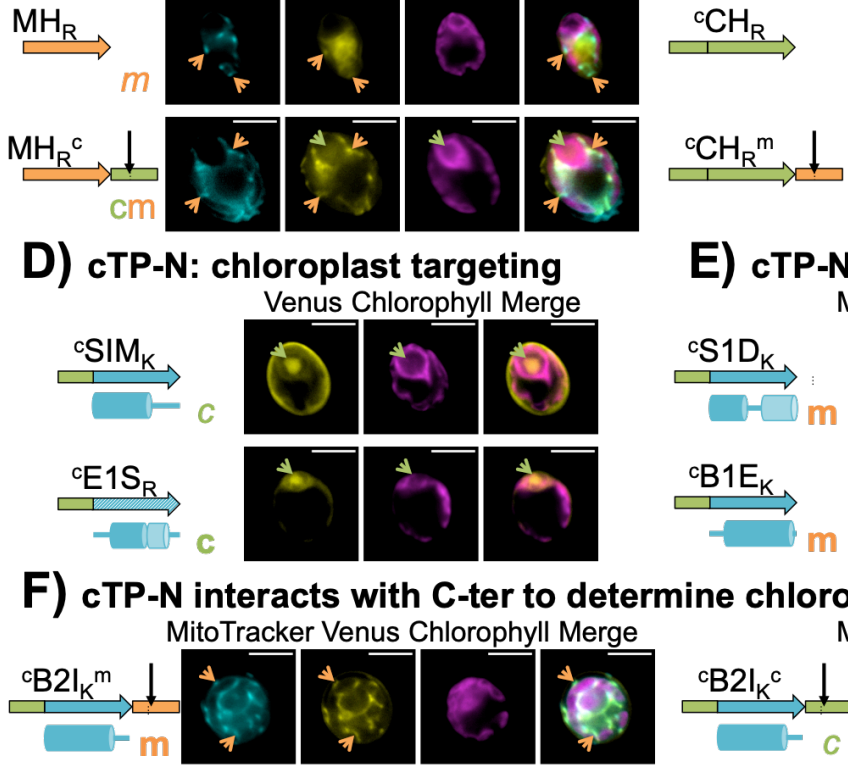

B) cTP-C: chloroplast targeting MitoTracker Venus Chlorophyll Merge
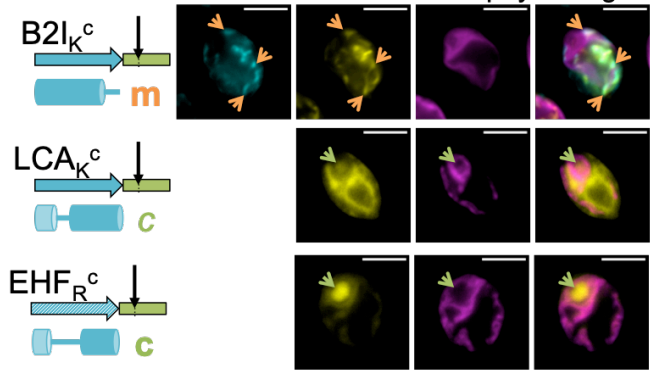

targeting in TP controls

Venus Chlorophyll Merge
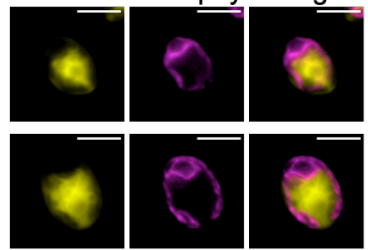

E) cTP-N compatible with mitochondria MitoTracker Venus Chlorophyll Merge

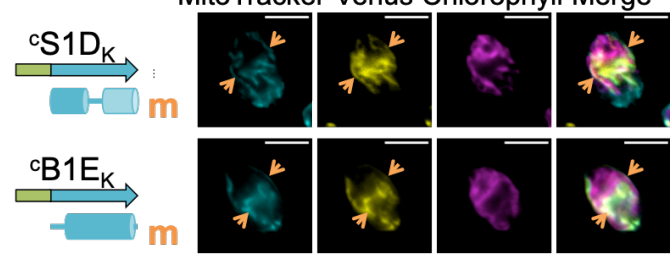

ine chloroplast localisation

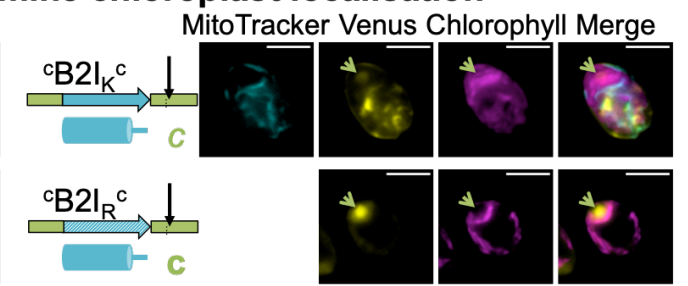

G) Combining CTP-N and CTP-C generates strong chloroplast determination MitoTracker Venus Chlorophyll Merge
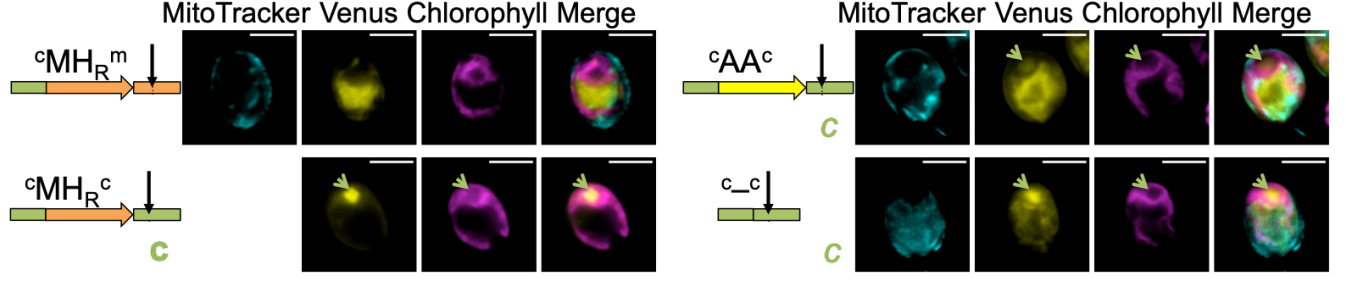

843 Figure 5. TP N- and C-termini enable chloroplast targeting by HA-RAMPs.

844 Exemplary epifluorescence images are shown as in Fig. 3; same convention as in

845 Fig. 4 for Venus localisation. Note that cTP-C contains a pyrenoid localization

846 motif $^{70}$, explaining the particularly pronounced Venus accumulation in the

847 pyrenoid in constructs carrying this element. The result for $B 2 \mathrm{I}_{\mathrm{K}}^{\mathrm{c}}$ in (B) confirms 
A) cTPs are longer

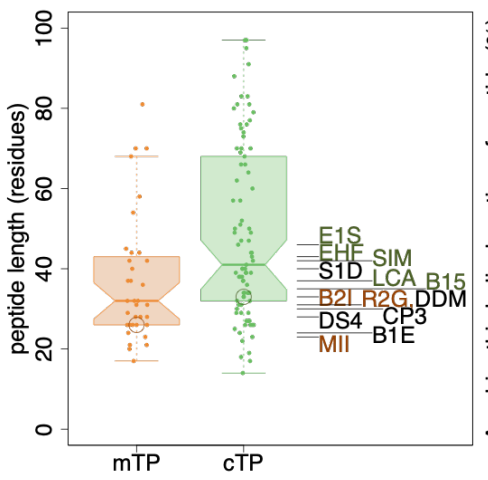

B) cTPs are less helical

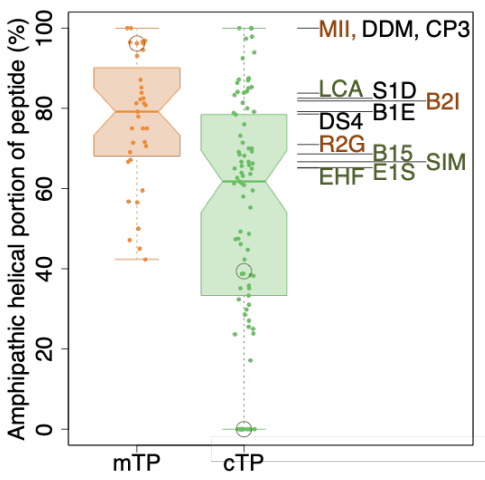

C) main helix start

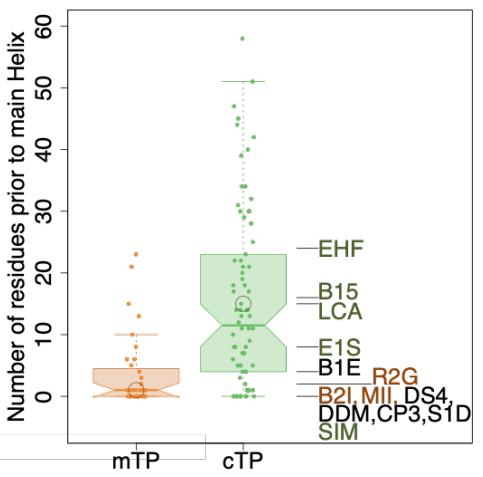

851 Figure 7. Chloroplast and mitochondrial targeting HA-RAMPs match cTPs

852 and mTPs respectively. For salient properties, Chlamydomonas mTPs and cTPs

853 are compared to our 13 HA-RAMPs. TP distributions are shown as boxplots

854 (center line: median; box limits: upper and lower quartiles; whiskers: $\min / \mathrm{max}$

855 values within $1.5 x$ interquartile range), coloured points represent individual

856 peptides. The position of the CAG2-mTP and RBCA-cTP is circled in each graph.

857 The non-zero value for RBCA-cTP in (B) and the single circle in (C) report on the 858 amphipathic helix established by an NMR-study ${ }^{11}$, since no helix could be 859 predicted by our approach. HA-RAMPs are colour-coded by preferred targeting:

860 orange $=$ mt-set; green $=$ cp-set (cf. Fig. 2). A) cTPs are significantly longer than 861 mTPs ( $p=0.0017)$, and $c p-s e t$ are longer than mt-set HA-RAMPs $(p=0.0184)$. B)

862 Helices make up a significantly smaller fraction of cTPs than mTPs $(\mathrm{p}<0.0001)$,

863 and HA-RAMPs show a similar trend (0.1122). C) cTPs contain significantly

864 longer sequence stretches upstream of the longest predicted helix than mTPs $865(\mathrm{p}<0.0001)$, as do cp-set compared to mt-set HA-RAMPs $(\mathrm{p}=0.0205)$. Reported $\mathrm{p}$ 866 values were obtained through two-way t-tests for TPs and one-way t-tests for 867 HA-RAMPs. 


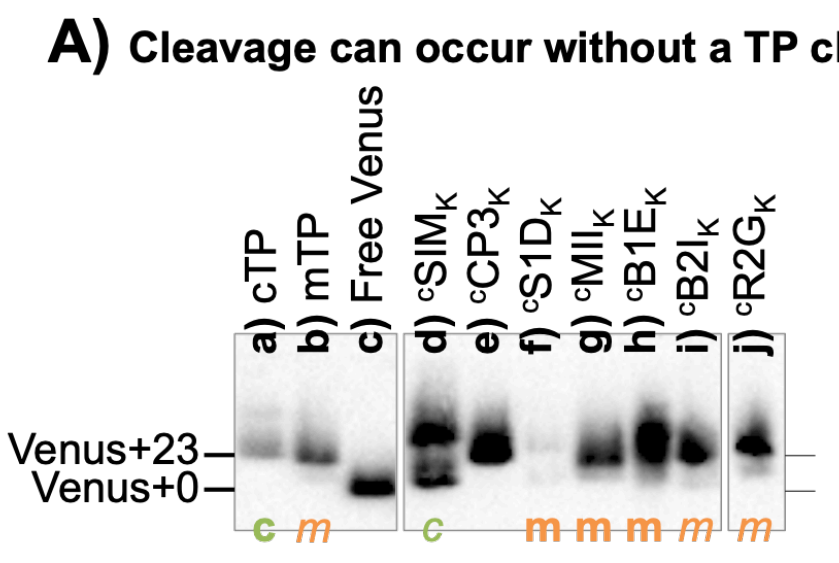

\section{B) TP cleavage sites are recognized in both organelles}

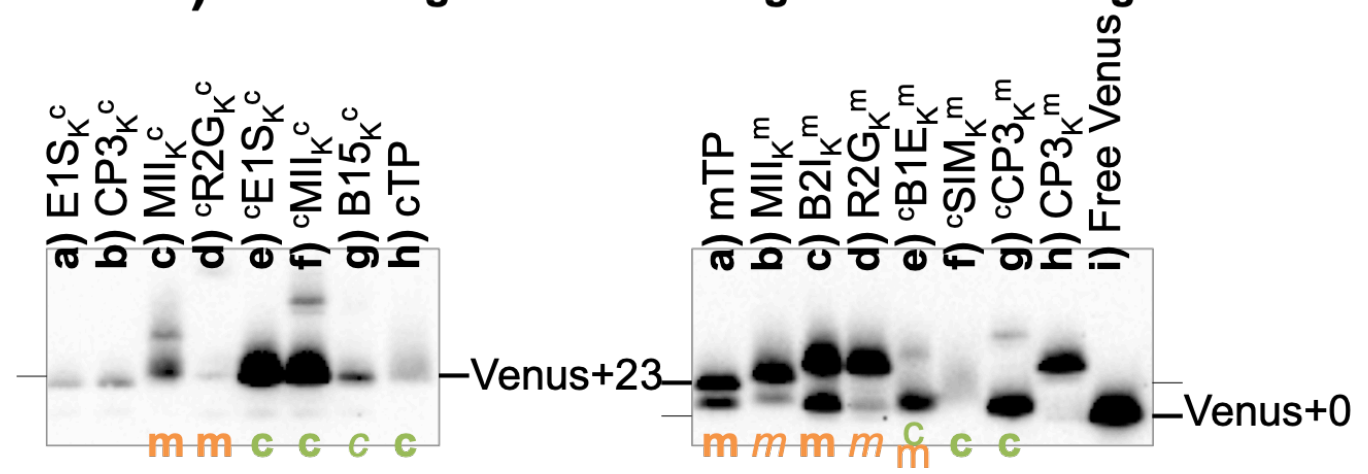

Blot 1: cTP-C

Blot 2: mTP-C

C) Increase in targeting upon $K \rightarrow R$ is visible in cleavage

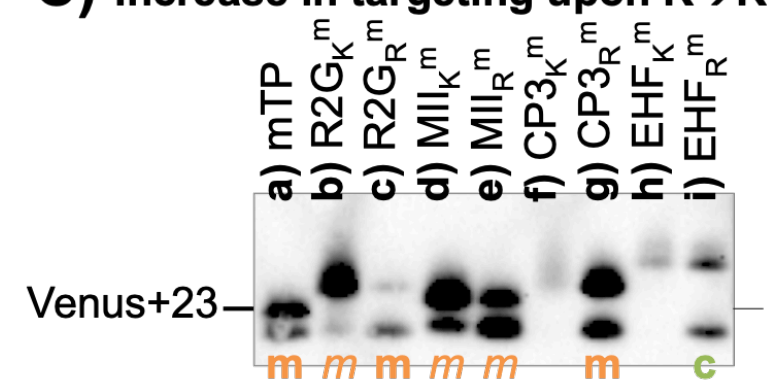

869 Figure 8. Import is associated with maturation of the preprotein. Western

870 Blots used an $\alpha$-FLAG antibody on selected constructs as indicated above blots.

871 Where a construct was interpreted as generating reporter localization by

872 fluorescence microscopy (cf. Fig. 2, Sup. Fig. 1-18) in mitochondria or

873 chloroplast, this is indicated by an orange ' $m$ ' or a green ' $c$ ' respectively, in bold

874 for full targeting or in italics for partial targeting. In A) some lanes were spliced

875 for clarity; the uncropped blot is provided in Sup. Fig. 26. 


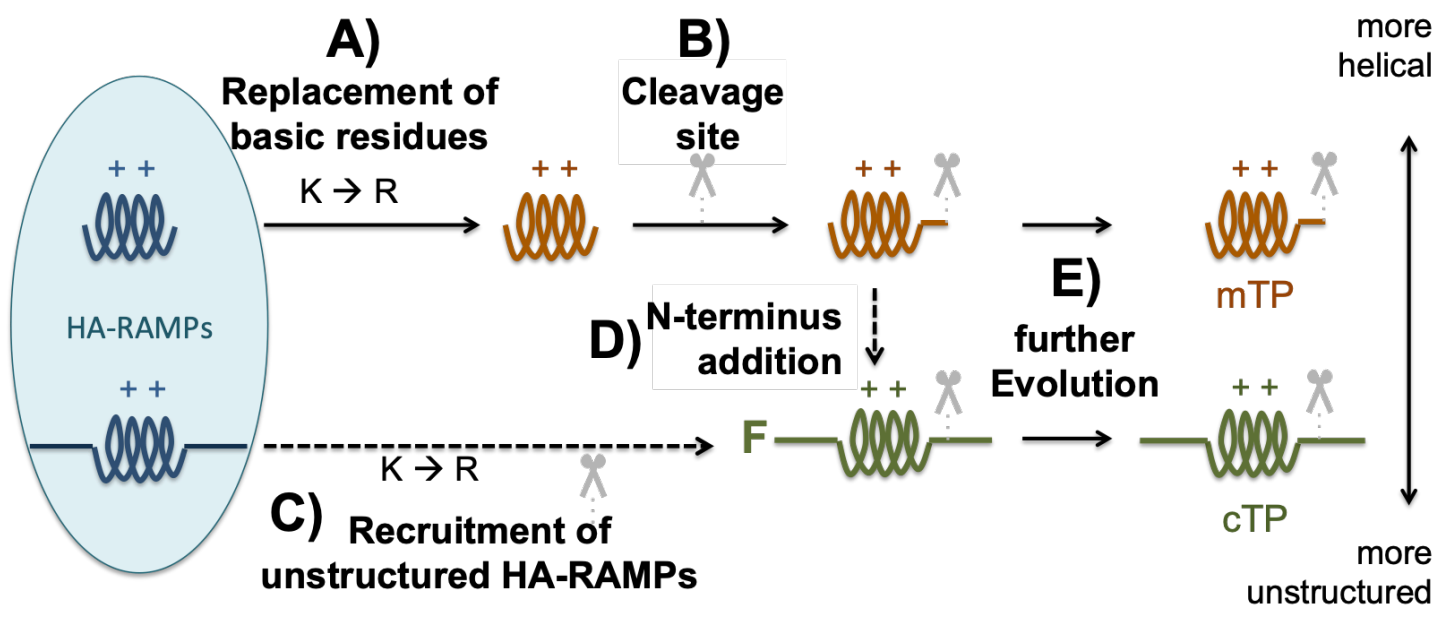

876

877 Figure 9. A proposed progression for the evolution of targeting peptides

878 from antimicrobial origins. A) HA-RAMPs would have first changed $\mathrm{K}$ to $\mathrm{R}$ and

879 B) acquired a cleavage site to become mTPs. To generate cTPs, either C) more 880 unstructured HA-RAMPs were recruited directly to become cTPs by undergoing

881 a K to R shift and acquiring a cleavage site separately, or D) mTPs acquired an N-

882 terminal non-helical domain. Early cTP N-termini likely interacted with TOC75

883 POTRA domains via a motif starting with $\mathrm{F}^{39,40}$. E) Further evolution would have

884 reinforced the differences between cTPs and mTPs to limit mis-targeting. 
bioRxiv preprint doi: https://doi.org/10.1101/2021.12.03.471120; this version posted December 3, 2021. The copyright holder for this

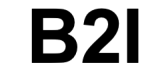

A

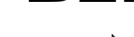

Strain 1
MitoTrackerVenus Chlorophy

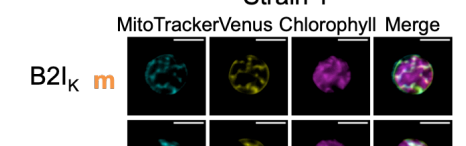

B $\Longrightarrow$

C

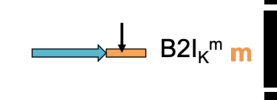

D $\Longleftrightarrow \mathrm{B} 2 \mathrm{I}_{\mathrm{R}}^{\mathrm{m}} \mathrm{m}$

E

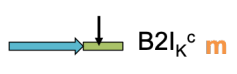

$\mathbf{F} \Longrightarrow \mathrm{B} 2 \mathrm{I}_{\mathrm{R}}^{\mathrm{c}} \mathrm{m}$

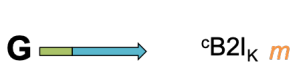

$\mathbf{H} \rightleftharpoons \quad{ }^{\mathrm{C}} \mathrm{B} 2 \mathrm{I}_{\mathrm{R}} \mathrm{m}$

$\mathrm{I} \rightleftharpoons \mathrm{CB} 2 \mathrm{~K}_{\mathrm{k}}^{\mathrm{m}} \mathrm{m}$

$\mathbf{J} \rightleftharpoons{ }^{\mathrm{CB} 2} 21_{\mathrm{R}}^{\mathrm{m}} \mathrm{Cm}$

$\mathbf{K} \rightleftharpoons b^{\mathrm{C}} \mathrm{B} 2 \mathrm{~K}^{\mathrm{c}}$

$\mathbf{L} \rightleftharpoons \mathrm{CB} 21_{\mathrm{R}}{ }^{\mathrm{C}} \mathrm{C}$
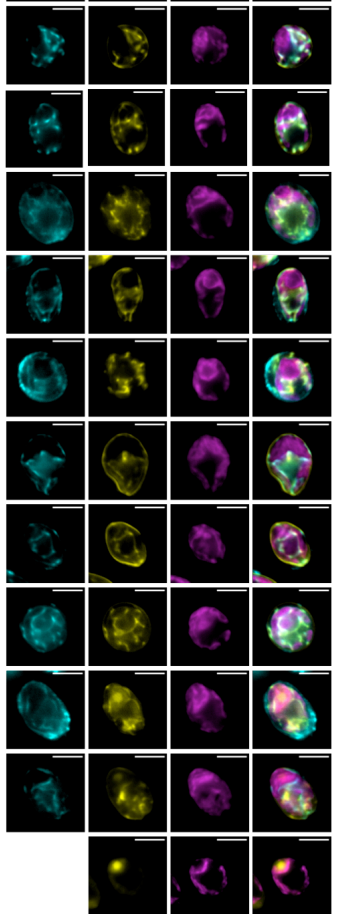
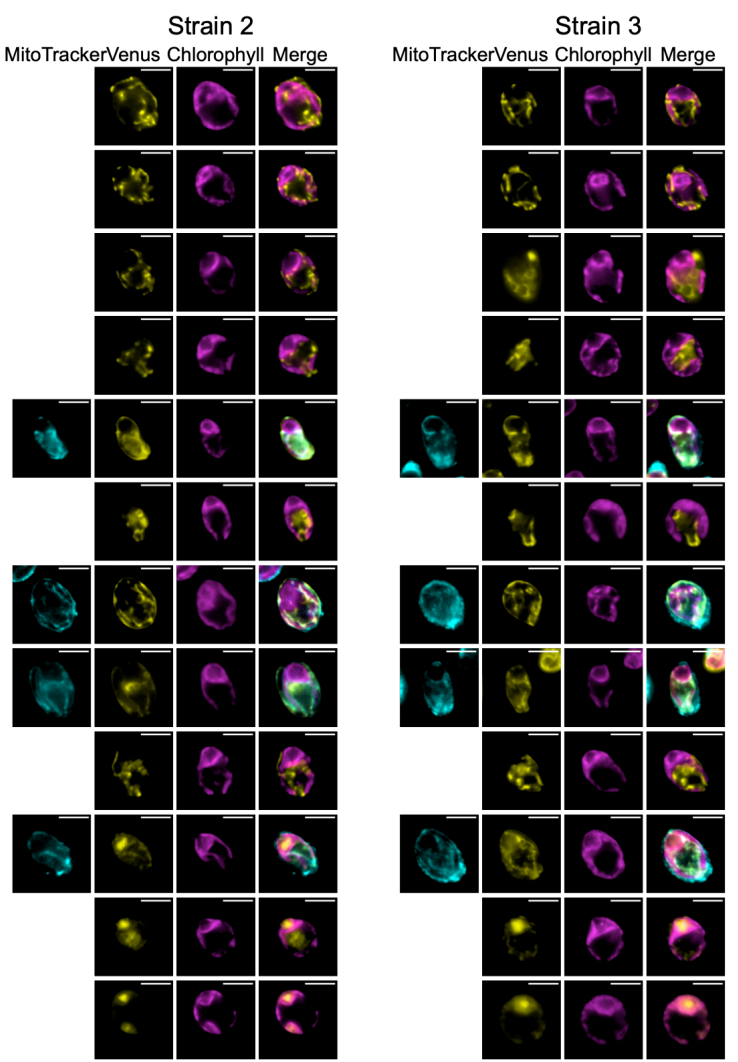

887 Supplementary Fig. 1. Microscopy for biological replicates of Brevinin 2ISb.

888 Three independent transformant lines (Strains 1-3) are shown for each

889 combination of modifications (A-L), represented by a cartoon and a shorthand

890 description (cf. Fig. 2). Where a construct was interpreted as generating reporter

891 localization in mitochondria or chloroplast, this is indicated by an orange ' $m$ ' or a

892 green 'c' respectively, in bold for full targeting or in italics for partial targeting.

893 Scale bars are $5 \mu \mathrm{m}$. 
bioRxiv preprint doi: https://doi org/10.1101/2021.12 03.471120: this version posted December 3, 2021. The copyright holder for this

\section{MII}

A

$\mathbf{A} \rightleftharpoons \mathrm{MII}_{\mathrm{K}} \mathrm{m}$

B

C $\Longrightarrow \mathrm{MII}_{\mathrm{K}} \mathrm{m}^{\mathrm{m}}$

D $\Longleftrightarrow \mathrm{MII}_{\mathbf{R}} \mathrm{m}^{\mathrm{m}}$

E $\Longrightarrow \mathrm{MII}_{\mathrm{K}}^{\mathrm{c}} \mathrm{m}$

$\mathbf{F}$

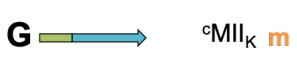

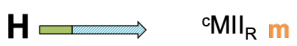

$\mathbf{1} \mathrm{cMIII}_{\mathrm{K}}^{\mathrm{m}} \mathrm{m}$

$\mathbf{J} \rightleftharpoons \frac{1}{\mathrm{c} M I_{\mathrm{R}} \mathrm{m} \mathrm{CH}}$

$\mathbf{K} \rightleftharpoons \mathrm{CMII}_{\mathrm{K}}^{\mathrm{c}} \mathrm{c}$

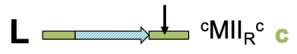

Strain 1 MitoTrackerVenus Chlorophyll Merge
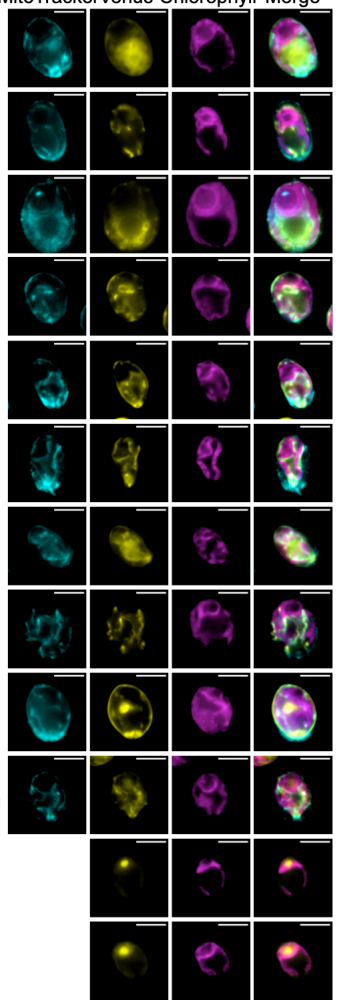

Strain 2

MitoTrackerVenus Chlorophyll Merge
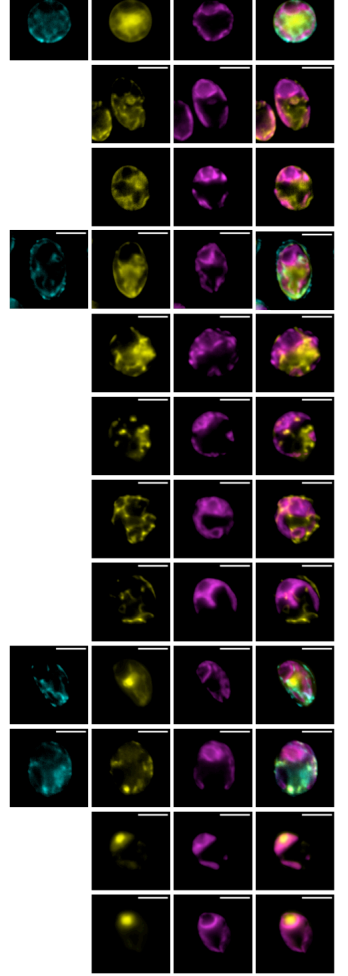

Strain 3

MitoTrackerVenus Chlorophyll Merge
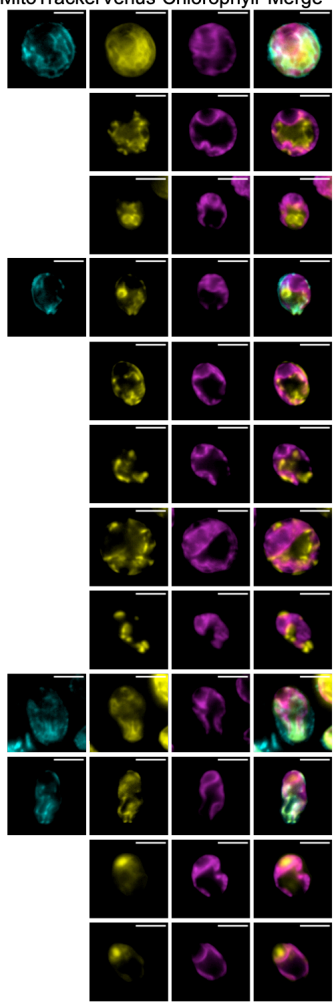

895 Supplementary Fig. 2. Microscopy for biological replicates of Magainin 2.

896 Three independent transformant lines (Strains 1-3) are shown for each

897 combination of modifications (A-L), represented by a cartoon and a shorthand

898 description (cf. Fig. 2). Where a construct was interpreted as generating reporter

899 localization in mitochondria or chloroplast, this is indicated by an orange ' $m$ ' or a

900 green 'c' respectively, in bold for full targeting or in italics for partial targeting.

901 Scale bars are $5 \mu \mathrm{m}$. 
bioRxiv preprint doi: https://doi org/10.1101/2021.12 03.471120; this version posted December 3, 2021. The copyright holder for this

R2G

A

$\Rightarrow R 2 G_{k}$

B

C

D $\rightleftharpoons \mathrm{R} 2 \mathrm{G}_{\mathrm{R}} \mathrm{m}$

$E \rightleftharpoons{\mathrm{R} 2 \mathrm{G}_{\mathrm{K}} \mathrm{c}}^{\mathrm{c}}$

$\mathbf{F}$

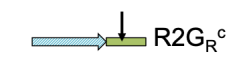

$\mathbf{G} \rightleftharpoons{ }^{\mathrm{C}} 2 \mathrm{G}_{\mathrm{K}} \mathrm{m}$

$\mathbf{H} \rightleftharpoons \quad{ }^{\mathrm{C} R} 2 \mathrm{G}_{\mathrm{R}} \mathrm{m}$

I $\rightleftharpoons{ }^{\mathrm{c} R} 2 \mathrm{G}_{\mathrm{K}}^{\mathrm{m} m}$

$\mathbf{J} \rightleftharpoons \stackrel{\downarrow}{\longrightarrow} \mathrm{R} 2 \mathrm{G}_{\mathrm{R}} \mathrm{m}_{\mathrm{m}}$

$\mathbf{K} \rightleftharpoons d^{\mathrm{c} R} 2 \mathrm{G}_{\mathrm{K}}^{\mathrm{c} m}$

$\mathbf{L} \rightleftharpoons{ }^{\mathrm{c} R} 2 \mathrm{G}_{\mathrm{R}}^{\mathrm{c} m}$
Strain 1

MtoTrackerVenus Chlorophyll Merge
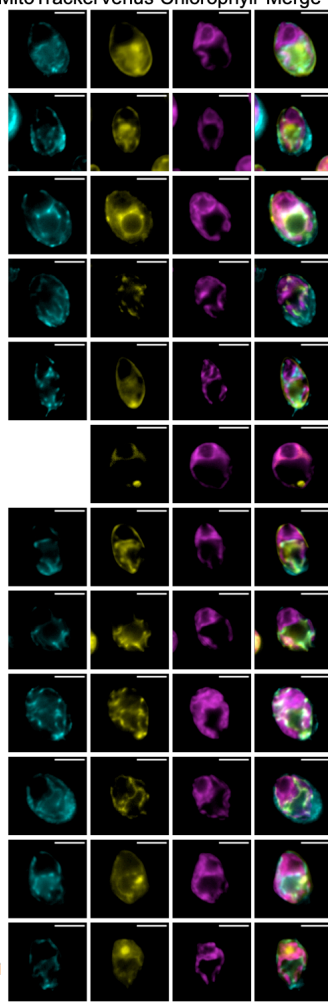

Strain 2

MitoTrackerVenus Chlorophyll Merge
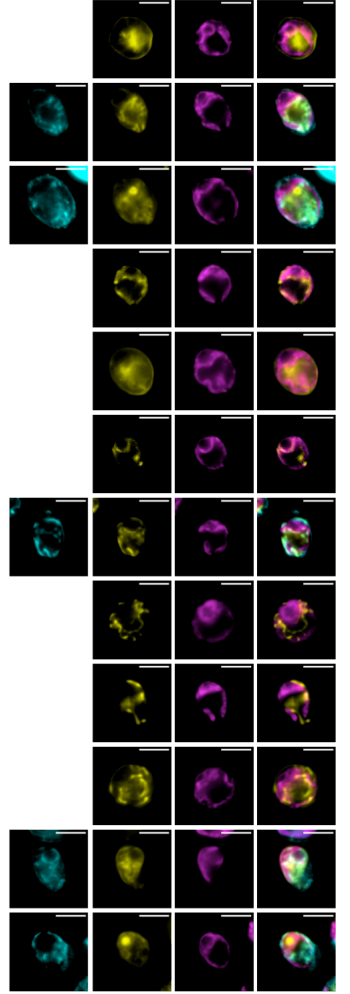

Strain 3
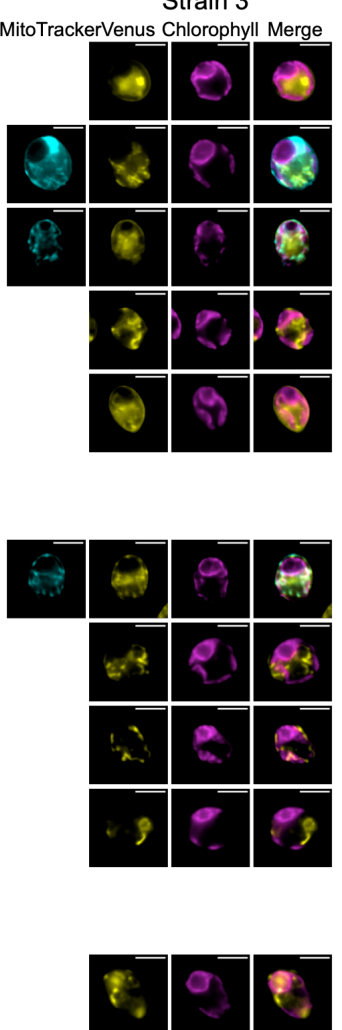

903

Supplementary Fig. 3. Microscopy for biological replicates of Ranatuerin

904 2G. Three independent transformant lines (Strains 1-3) are shown for each

905 combination of modifications (A-L), represented by a cartoon and a shorthand

906 description (cf. Fig. 2). Where a construct was interpreted as generating reporter

907 localization in mitochondria or chloroplast, this is indicated by an orange ' $m$ ' or a

908 green ' $c$ ' respectively, in bold for full targeting or in italics for partial targeting.

909 Scale bars are $5 \mu \mathrm{m}$. 
bioRxiv preprint doi: https://doi org/10.1101/2021.12 03.471120: this version posted December 3, 2021. The copyright holder for this

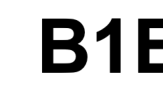

A

B $\Longrightarrow B 1 E_{R}$

$\mathrm{C} \rightleftharpoons \mathrm{B} 1 \mathrm{E}_{\mathrm{K}}^{\mathrm{m}}$

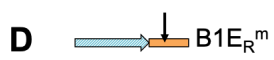

$\mathrm{E} \Longrightarrow \mathrm{D} 1 \mathrm{E}_{\mathrm{K}}^{\mathrm{c}}$

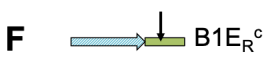

$\mathbf{G} \rightleftharpoons{ }^{\mathrm{BB} 1 \mathrm{E}_{\mathrm{K}} \mathrm{m}}$

$\mathbf{H} \Longrightarrow{ }^{\mathrm{C} B 1 E_{\mathrm{R}} \mathrm{m}}$

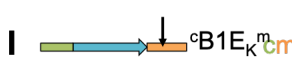

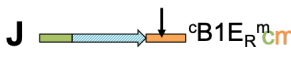

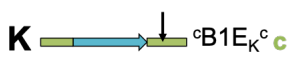

$\mathbf{L} \Longrightarrow b^{\mathrm{CB} 1 \mathrm{E}_{\mathrm{R}}{ }^{\mathrm{C}} \mathrm{C}}$
Strain 1

MitoTrackerVenus Chlorophyll Merge
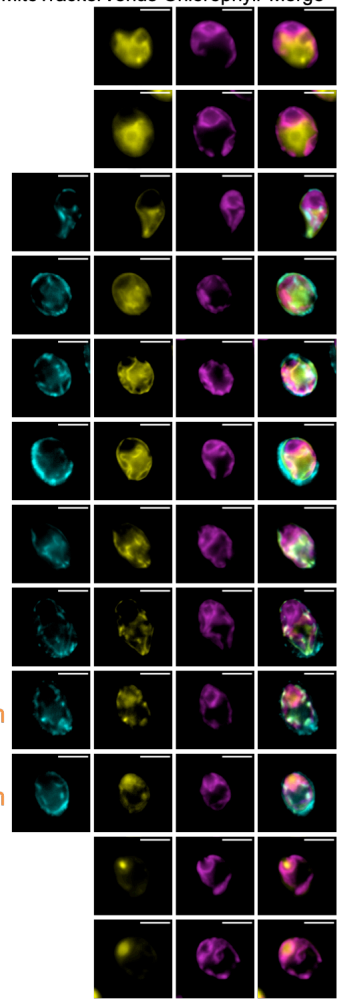

Strain 2

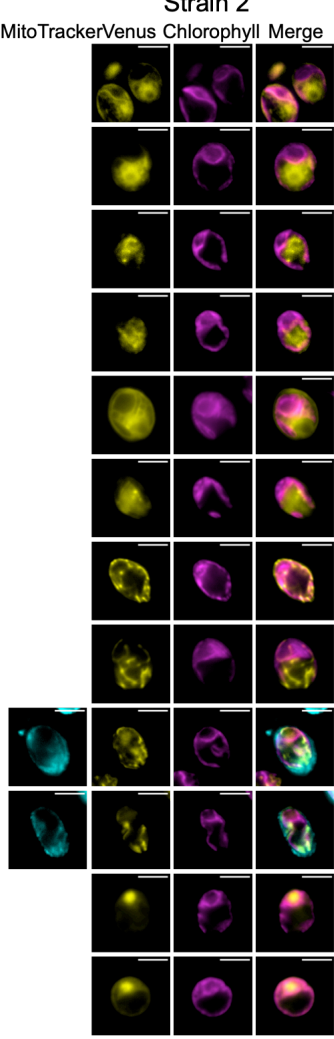

Strain 3

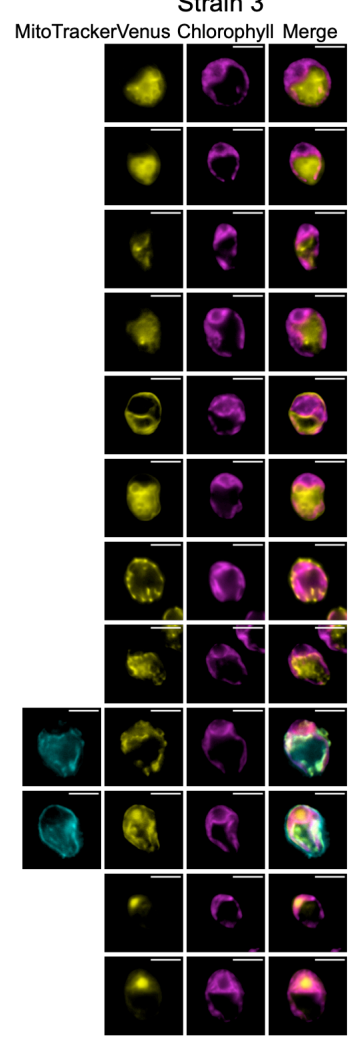

911 Supplementary Fig. 4. Microscopy for biological replicates of Brevinin 1E.

912 Three independent transformant lines (Strains 1-3) are shown for each

913 combination of modifications (A-L), represented by a cartoon and a shorthand

914 description (cf. Fig. 2). Where a construct was interpreted as generating reporter

915 localization in mitochondria or chloroplast, this is indicated by an orange ' $\mathrm{m}$ ' or a

916 green 'c' respectively, in bold for full targeting or in italics for partial targeting.

917 Scale bars are $5 \mu \mathrm{m}$. 


\section{DS4}

$\mathrm{A} \rightleftharpoons \mathrm{DS}_{\mathrm{K}}$

B $\Longrightarrow \mathrm{DS}_{\mathrm{R}}$

C $\Longrightarrow \mathrm{DS4}_{\mathrm{K}}{ }^{\mathrm{m}}$

D $\Longrightarrow \mathrm{DS4}_{\mathrm{R}} \mathrm{m}^{\mathrm{m}}$

E $\Longrightarrow \mathrm{DS}_{\mathrm{K}}{ }^{\mathrm{c}}$

F $\longleftrightarrow \mathrm{DS4}_{\mathrm{R}}{ }^{\mathrm{c}}$

$\mathbf{G} \rightleftharpoons{ }^{\mathrm{cDS}} 4_{\mathrm{K}}$

$\mathbf{H} \rightleftharpoons{ }^{\mathrm{c} D S 4} 4_{\mathrm{R}}$

$\mathbf{2} \downarrow^{\mathrm{CDDS} 4} \mathrm{~K}^{\mathrm{m}}$

$\mathbf{J} \rightleftharpoons d^{\mathrm{c} D S 4}{ }_{\mathrm{R}}^{\mathrm{m}}$

$\mathrm{K} \rightleftharpoons \downarrow^{\mathrm{c} D S 4} \mathrm{~K}^{\mathrm{c}}$

$\mathbf{L} \mathrm{CDS4}_{\mathrm{R}}^{\mathrm{c}}$
Strain 1

MitoTrackerVenus Chlorophyll Merge
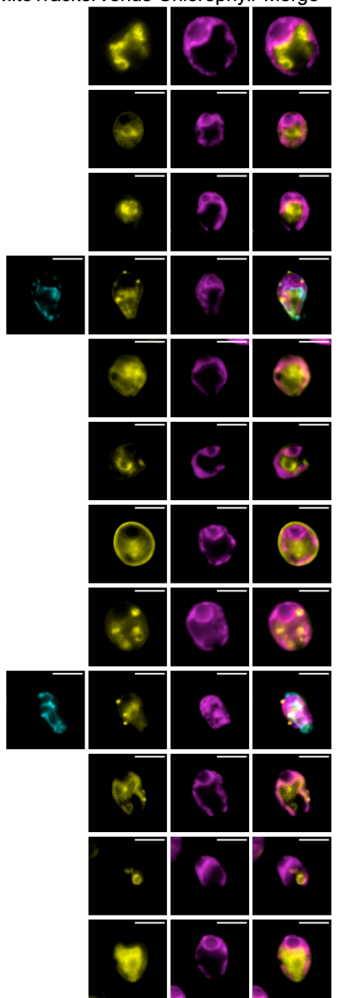

Strain 2

MitoTrackerVenus Chlorophyll Merge
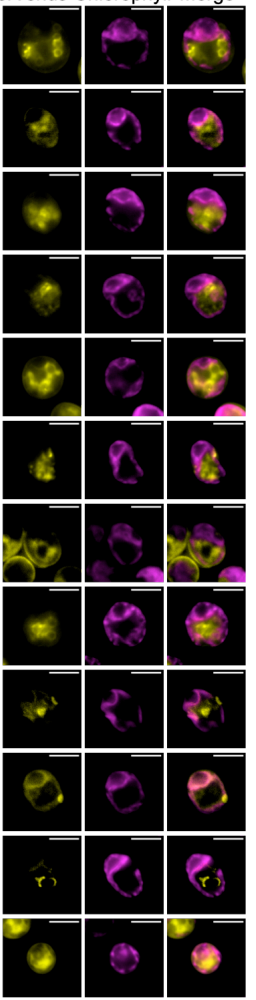

Strain 3

MitoTrackerVenus Chlorophyll Merge

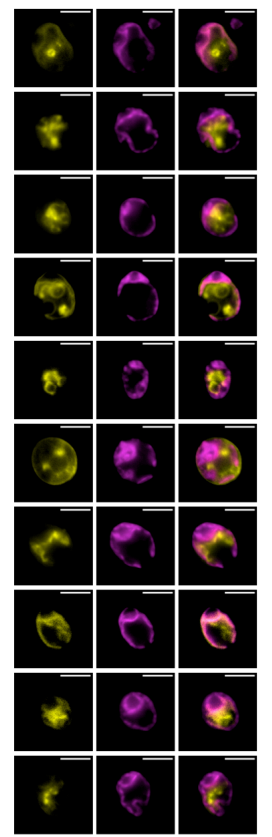

919 Supplementary Fig. 5. Microscopy for biological replicates of Dermaseptin

920 S4. Three independent transformant lines (Strains 1-3) are shown for each

921 combination of modifications (A-L), represented by a cartoon and a shorthand

922 description (cf. Fig. 2). Where a construct was interpreted as generating reporter

923 localization in mitochondria or chloroplast, this is indicated by an orange ' $\mathrm{m}$ ' or a

924 green ' $c$ ' respectively, in bold for full targeting or in italics for partial targeting.

925 Scale bars are $5 \mu \mathrm{m}$. 


\section{DDM}

A $\Longrightarrow \mathrm{DDM}_{\mathrm{K}}$

B $\Longrightarrow \mathrm{DDM}_{\mathrm{R}}$

C $\Longrightarrow \frac{1}{D_{D D M}}{ }^{\mathrm{m}}$

D $\longleftrightarrow \mathrm{DDM}_{\mathrm{R}}^{\mathrm{m}}$

E $\Longrightarrow \mathrm{DDM}_{\mathrm{K}} \mathrm{c}$

$\mathbf{F}$

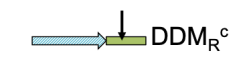

$\mathbf{G} \rightleftharpoons{ }^{c} \mathrm{DDM}_{\mathrm{K}}$

$\mathbf{H} \stackrel{{ }^{c} \mathrm{DDM}}{\mathrm{R} m}$

$\mathbf{1} \rightleftharpoons d^{\mathrm{c} D D M} \mathrm{~K}^{\mathrm{m}}$

$\mathbf{J} \rightleftharpoons{ }^{\mathrm{C} D D M} \mathrm{~m}_{\mathrm{R}}$

$\mathbf{K} \rightleftharpoons \operatorname{cDDM}^{\mathrm{C}} \mathrm{C}$

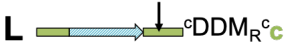

Strain 1

MitoTrackerVenus Chlorophyll Merge

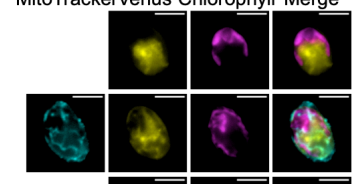

A. 0
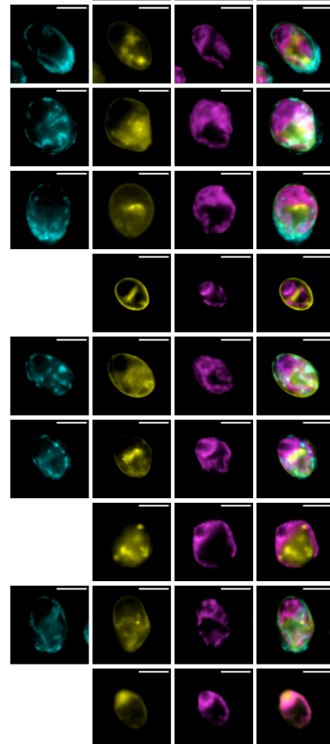

Strain 2

MitoTrackerVenus Chlorophyll Merge
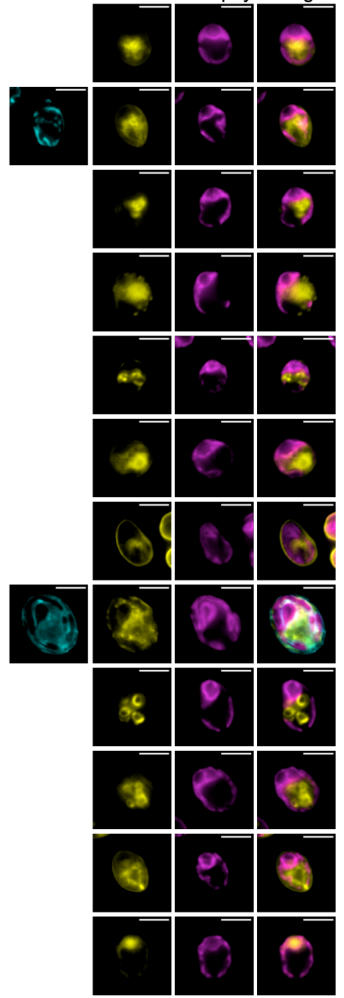

Strain 3

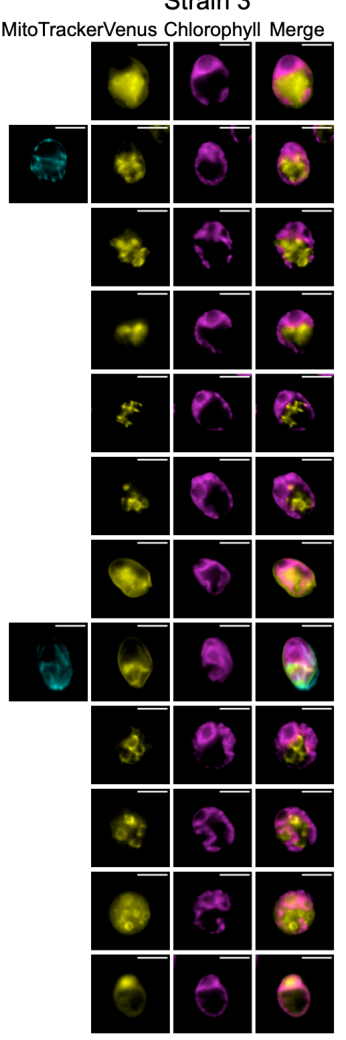

927 Supplementary Fig. 6. Microscopy for biological replicates of

928 Dermadistinctin M. Three independent transformant lines (Strains 1-3) are

929 shown for each combination of modifications (A-L), represented by a cartoon

930 and a shorthand description (cf. Fig. 2). Where a construct was interpreted as

931 generating reporter localization in mitochondria or chloroplast, this is indicated

932 by an orange ' $m$ ' or a green ' $c$ ' respectively, in bold for full targeting or in italics

933 for partial targeting. Scale bars are $5 \mu \mathrm{m}$. 


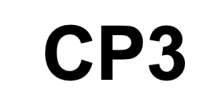$$
\text { A }
$$

Strain 1

MitoTrackerVenus Chlorophyll Merge
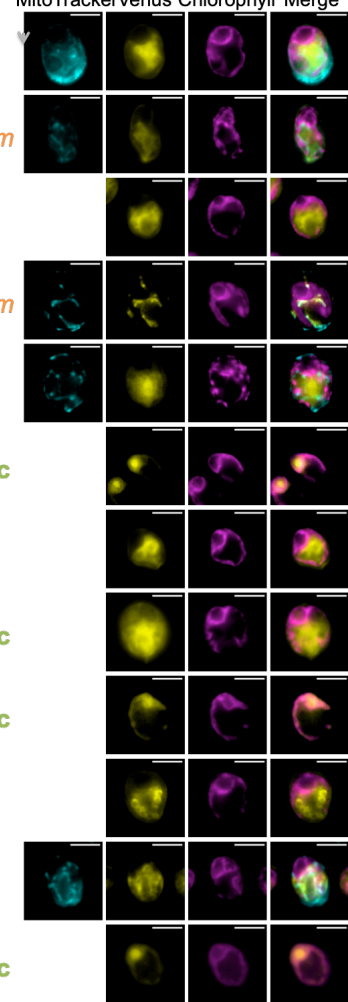

Strain 2

MitoTrackerVenus Chlorophyll Merge
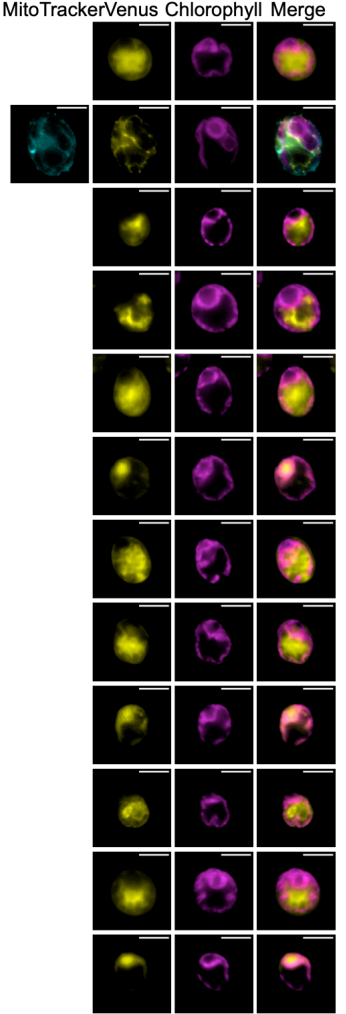

Strain 3

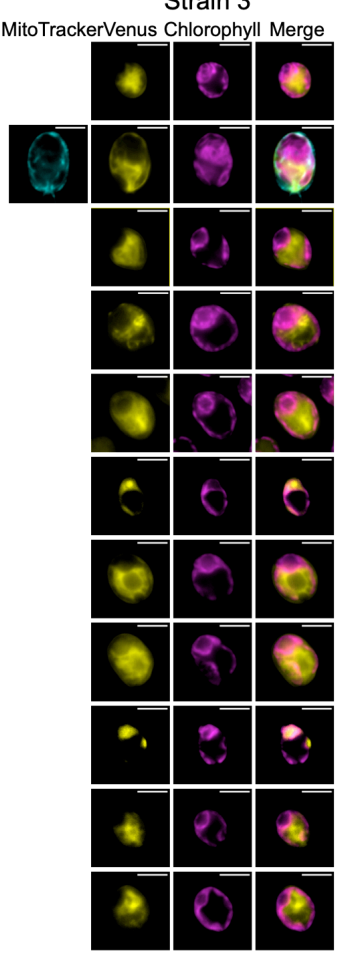

935 Supplementary Fig. 7. Microscopy for biological replicates of Cecropin P3.

936 Three independent transformant lines (Strains 1-3) are shown for each

937 combination of modifications (A-L), represented by a cartoon and a shorthand

938 description (cf. Fig. 2). Where a construct was interpreted as generating reporter

939 localization in mitochondria or chloroplast, this is indicated by an orange ' $\mathrm{m}$ ' or a

940 green ' $c$ ' respectively, in bold for full targeting or in italics for partial targeting.

941 Scale bars are $5 \mu \mathrm{m}$. 


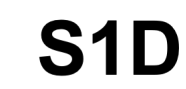

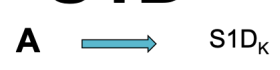

B $\Longrightarrow S 1 D_{R}$

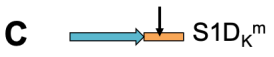

D $\Longrightarrow S 1 D_{R}{ }^{m}$

$E \rightleftharpoons S_{1} D_{K}^{c} c$

$\mathbf{F} \longrightarrow \mathrm{S}_{\mathrm{D}} \mathrm{D}_{\mathrm{R}}^{\mathrm{c} C}$

$\mathbf{G} \rightleftharpoons{ }^{c S} 1 D_{\mathrm{K}}$

$\mathbf{H} \rightleftharpoons \quad{ }^{\mathrm{S}} 1 \mathrm{D}_{\mathrm{R}} \mathrm{m}$

$\mathbf{1} \rightleftharpoons \mathrm{d} 1 \mathrm{D}_{\mathrm{k}}^{\mathrm{m}}$

$\mathbf{J} \rightleftharpoons c^{c} S 1 D_{R}^{m}$

$\mathbf{K} \rightleftharpoons d^{c} \mathrm{~S} 1 \mathrm{D}_{\mathrm{K}}^{\mathrm{c}} \mathrm{c}$

$\mathbf{L} \rightleftharpoons{ }^{c} \mathrm{~S} 1 \mathrm{D}_{\mathrm{R}}{ }^{\mathrm{C}} \mathrm{C}$
Strain 1

MitoTrackerVenus Chlorophyll Merge
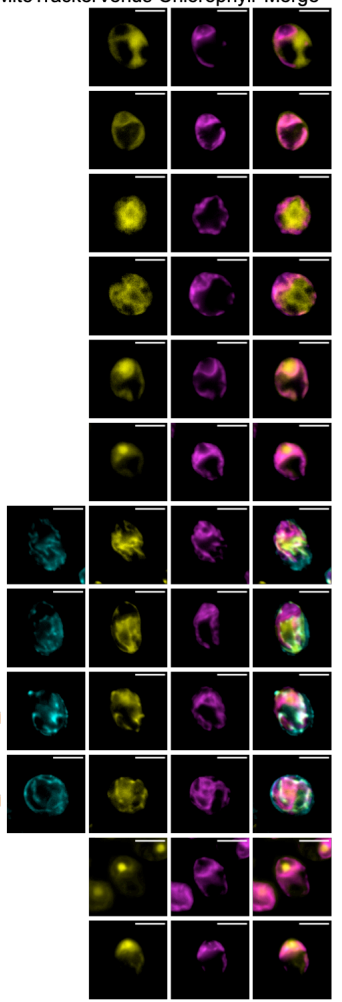

Strain 2

Mito TrackerVenus Chlorophyll Merge

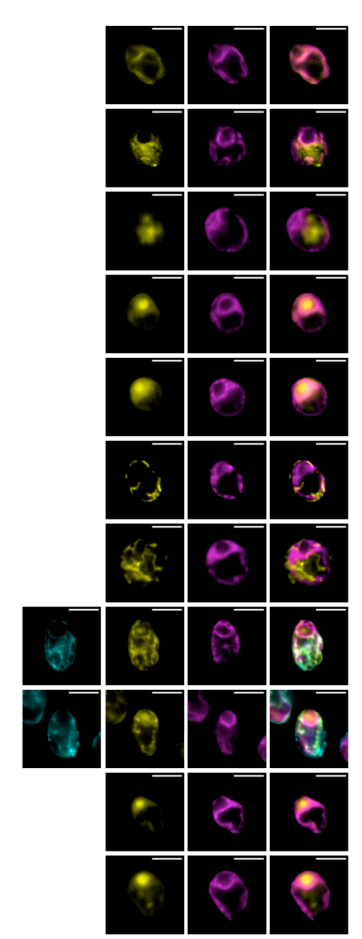

Strain 3

MitoTrackerVenus Chlorophyll Merge

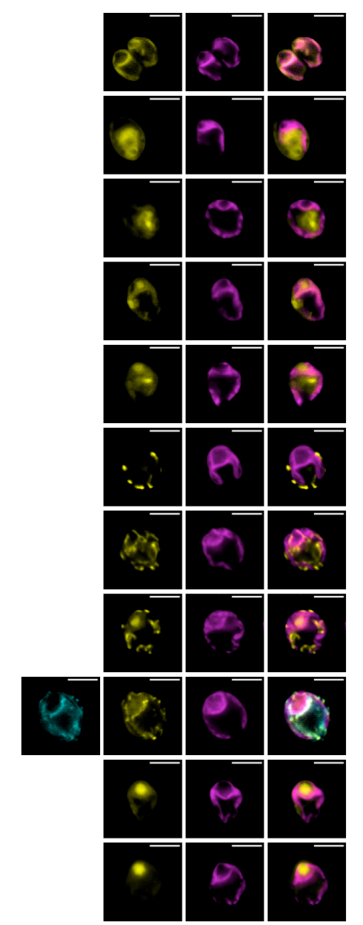

943 Supplementary Fig. 8. Microscopy for biological replicates of Sarcotoxin 1D.

944 Three independent transformant lines (Strains 1-3) are shown for each

945 combination of modifications (A-L), represented by a cartoon and a shorthand

946 description (cf. Fig. 2). Where a construct was interpreted as generating reporter

947 localization in mitochondria or chloroplast, this is indicated by an orange ' $\mathrm{m}$ ' or a

948 green ' $c$ ' respectively, in bold for full targeting or in italics for partial targeting.

949 Scale bars are $5 \mu \mathrm{m}$. 


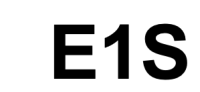

$\mathbf{A} \rightleftharpoons \mathrm{E}^{\mathrm{S}} \mathrm{S}_{\mathrm{K}}$

$B \quad \Longrightarrow \mathrm{E}^{\mathrm{B}} \mathrm{R}$

C $\Longrightarrow \mathrm{E}_{1} \mathrm{~S}_{\mathrm{K}}{ }^{\mathrm{m}}$

D $\rightleftarrows \mathrm{E} 1 \mathrm{~S}_{\mathrm{R}}^{\mathrm{m}} \mathrm{m}$

E $\Longrightarrow \mathrm{E}_{1} \mathrm{~S}_{\mathrm{K}}{ }^{\mathrm{c}}$

F

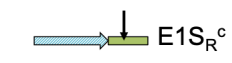

$\mathbf{G} \rightleftharpoons{ }^{\mathrm{c}} \mathrm{E} 1 \mathrm{~S}_{\mathrm{K}}$

$\mathbf{H} \rightleftharpoons \quad{ }^{\mathrm{C}} \mathrm{E} 1 \mathrm{~S}_{\mathrm{R}} \mathrm{c}$

$\mathbf{} \rightleftharpoons{ }^{\mathrm{c} E} \mathrm{~S}_{\mathrm{K}} \mathrm{m}_{\mathrm{c}}$

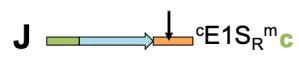

$\mathbf{} \rightleftharpoons b^{\mathrm{C} E} 1 \mathrm{~S}_{\mathrm{K}^{\mathrm{c}} \mathrm{C}}$

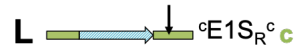

Strain 1

MitoTrackerVenus Chlorophyll Merge
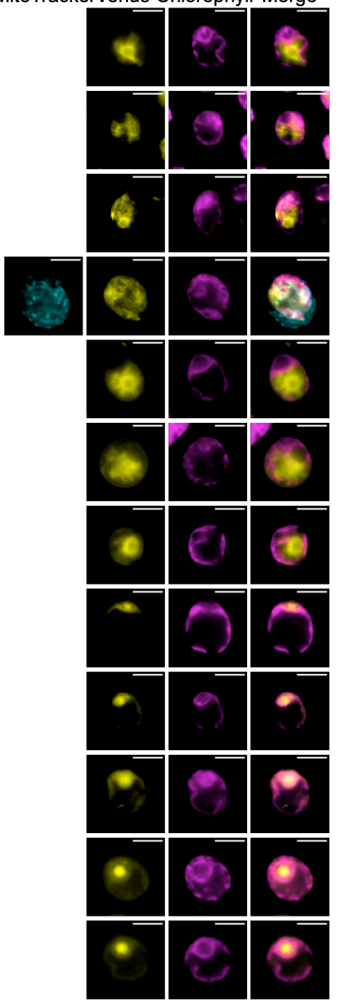

Strain 2

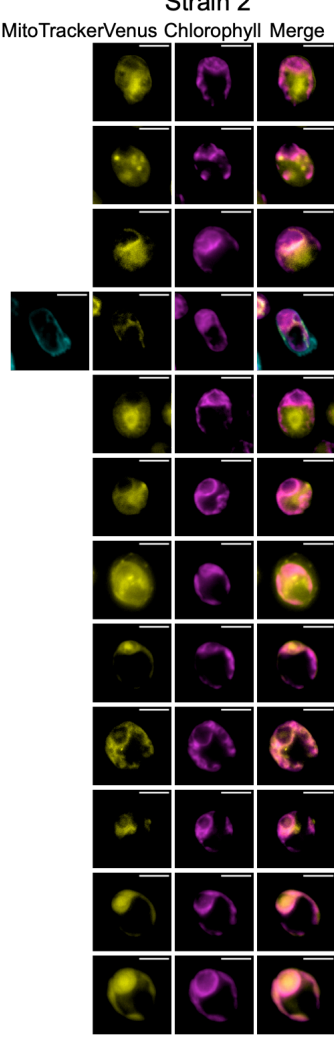

Strain 3
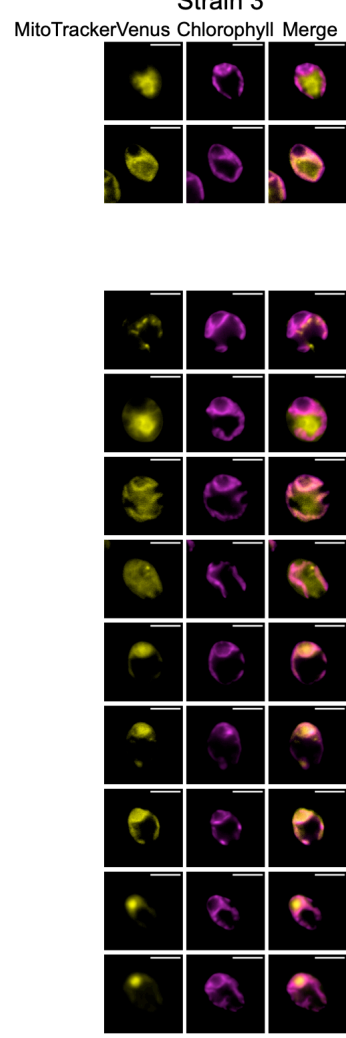

951 Supplementary Fig. 9. Microscopy for biological replicates of Esculentin 1S.

952 Three independent transformant lines (Strains 1-3) are shown for each

953 combination of modifications (A-L), represented by a cartoon and a shorthand

954 description (cf. Fig. 2). Where a construct was interpreted as generating reporter

955 localization in mitochondria or chloroplast, this is indicated by an orange ' $m$ ' or a

956 green ' $c$ ' respectively, in bold for full targeting or in italics for partial targeting.

957 Scale bars are $5 \mu \mathrm{m}$. 
bioRxiv preprint doi: https://doi.org/10.1101/2021.12.03.471120; this version posted December 3, 2021. The copyright holder for this

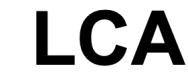

$A \rightleftharpoons$ LCA $_{K}$

B $\Longrightarrow \mathrm{LCA}_{\mathrm{R}}$

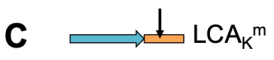

D $\Longrightarrow \mathrm{LCA}_{\mathrm{R}} \mathrm{m}^{\mathrm{m}}$

$E \rightleftharpoons \operatorname{LCA}_{K}{ }^{c} \mathrm{C}$

$\mathbf{F} \longleftrightarrow \mathrm{LCA}_{\mathrm{R}}^{\mathrm{c} C}$

$\mathbf{G} \rightleftharpoons \mathrm{cLCA}_{\mathrm{K}}$

$\mathbf{H} \rightleftharpoons{ }^{\mathrm{CLCA}} \mathrm{R}$

$\mathbf{}$

$\mathbf{J} \longmapsto d^{c} \mathrm{LCA}_{R}{ }^{\mathrm{m}}$

$\mathbf{K} \rightleftharpoons \mathrm{CLCA}_{\mathrm{K}}^{\mathrm{c} C}$

$\mathbf{L} \rightleftharpoons \mathrm{LCA}_{\mathrm{R}}^{\mathrm{c}} \mathrm{c}$
Strain 1

MitoTrackerVenus Chlorophyll Merge
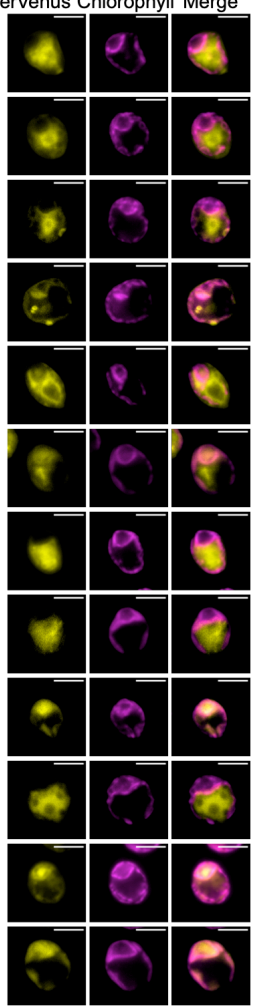

Strain 2

MitoTrackerVenus Chlorophyll Merge
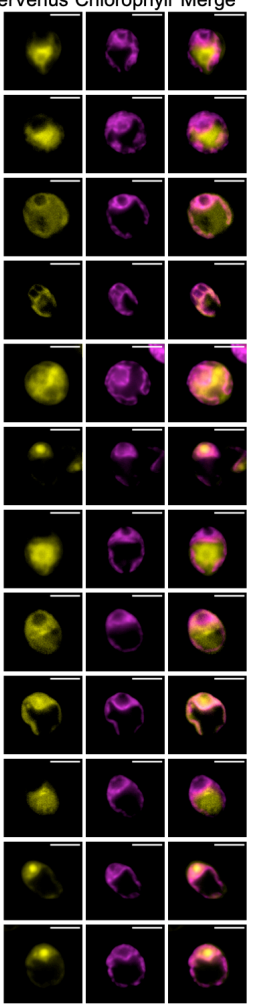

Strain 3

MitoTrackerVenus Chlorophyll Merge
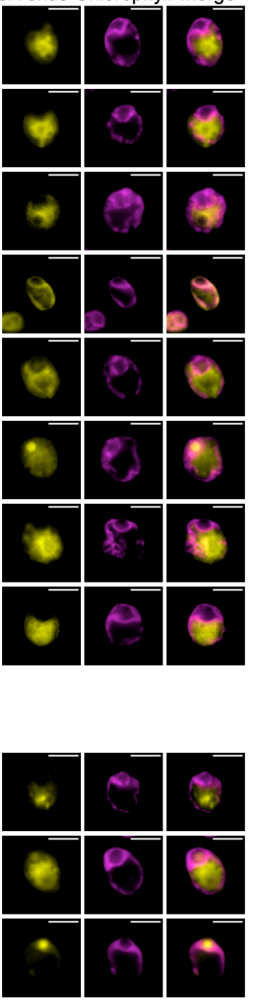

959 Supplementary Fig. 10. Microscopy for biological replicates of Leucocin A.

960 Three independent transformant lines (Strains 1-3) are shown for each

961 combination of modifications (A-L), represented by a cartoon and a shorthand

962 description (cf. Fig. 2). Where a construct was interpreted as generating reporter

963 localization in mitochondria or chloroplast, this is indicated by an orange ' $m$ ' or a

964 green ' $c$ ' respectively, in bold for full targeting or in italics for partial targeting.

965 Scale bars are $5 \mu \mathrm{m}$. 


\section{SIM}$$
\text { A }
$$

B

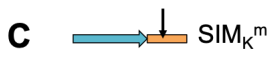

D $\Longleftrightarrow \operatorname{SIM}^{\mathrm{m}}$

E $\Longrightarrow \operatorname{SIM}_{\mathrm{K}}{ }^{\mathrm{C}}$

F $\longrightarrow \mathrm{SIM}_{\mathrm{R}}{ }^{\mathrm{C}}$

$\mathbf{G} \rightleftharpoons{ }^{c} \operatorname{SIM}_{\mathrm{K}} c$

$\mathbf{H} \longmapsto \quad{ }^{c} \operatorname{SIM}_{\mathrm{R}} \mathrm{c}$

I $\downarrow^{\mathrm{c} S I M} \mathrm{~K}_{\mathrm{K}}^{\mathrm{m}} \mathrm{C}$

$\mathbf{J} \downarrow^{c} \mathrm{SIM}_{R}{ }^{\mathrm{m}} \mathrm{c}$

$\mathbf{} \rightleftharpoons{ }^{c} \operatorname{SIM}_{\mathrm{K}}^{\mathrm{c} c}$

$\mathbf{L} d^{\mathrm{C}} \mathrm{SIM}_{\mathrm{R}}^{\mathrm{c} C}$
Strain 1

MitoTrackerVenus Chlorophyll Merge
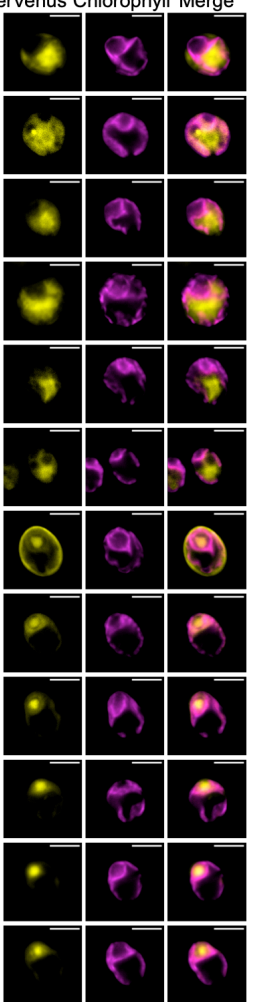

Strain 2

MitoTrackerVenus Chlorophyll Merge
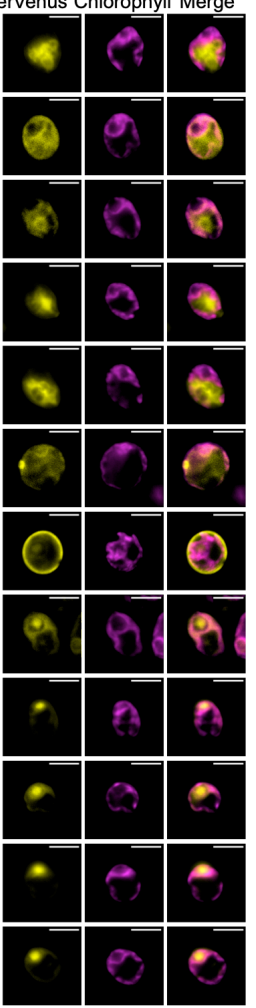

Strain 3

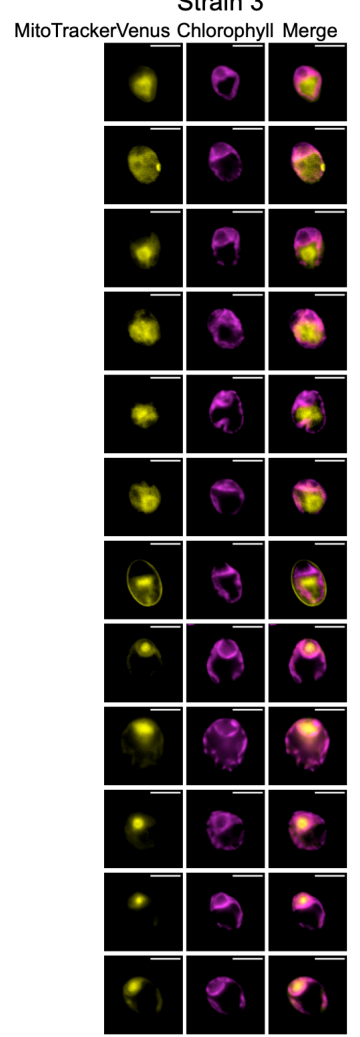

967 Supplementary Fig. 11. Microscopy for biological replicates of SI Moricin.

968 Three independent transformant lines (Strains 1-3) are shown for each

969 combination of modifications (A-L), represented by a cartoon and a shorthand

970 description (cf. Fig. 2). Where a construct was interpreted as generating reporter

971 localization in mitochondria or chloroplast, this is indicated by an orange ' $\mathrm{m}$ ' or a

972 green 'c' respectively, in bold for full targeting or in italics for partial targeting.

973 Scale bars are $5 \mu \mathrm{m}$. 


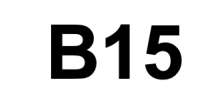

A

B

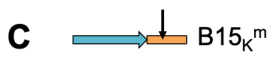

D $\Longrightarrow \mathrm{B}_{15}{ }_{\mathrm{R}}{ }^{\mathrm{m}}$

E $\Longrightarrow \mathrm{B} 15_{\mathrm{K}^{\mathrm{c}} \mathrm{c}}$

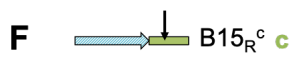

$\mathbf{G} \rightleftharpoons{ }^{\mathrm{CB} 15_{\mathrm{K}}}$

$\mathbf{H} \rightleftharpoons \quad{ }^{\mathrm{C} B} 15_{\mathrm{R}} \mathrm{c}$

$\mathrm{I} \rightleftharpoons{ }^{\mathrm{CB} 15} \mathrm{~K}^{\mathrm{m}} \mathrm{c}$

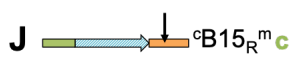

$\mathbf{K} \rightleftharpoons{ }^{\mathrm{C} B 15} \mathrm{~K}^{\mathrm{c} c}$

$\mathbf{L} \downarrow^{\mathrm{C} B 15}{ }_{\mathrm{R}}^{\mathrm{C} C}$
Strain 1

MitoTrackerVenus Chlorophyll Merge
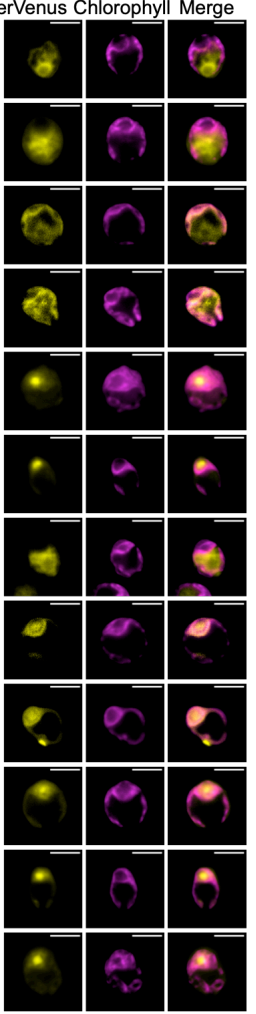

Strain 2

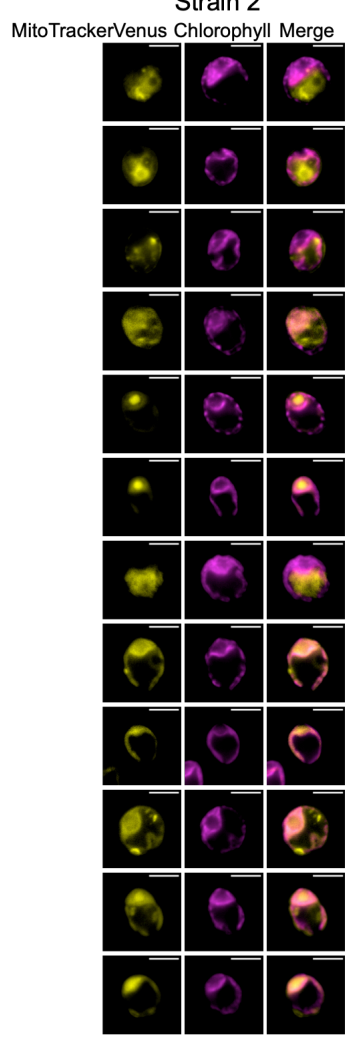

Strain 3

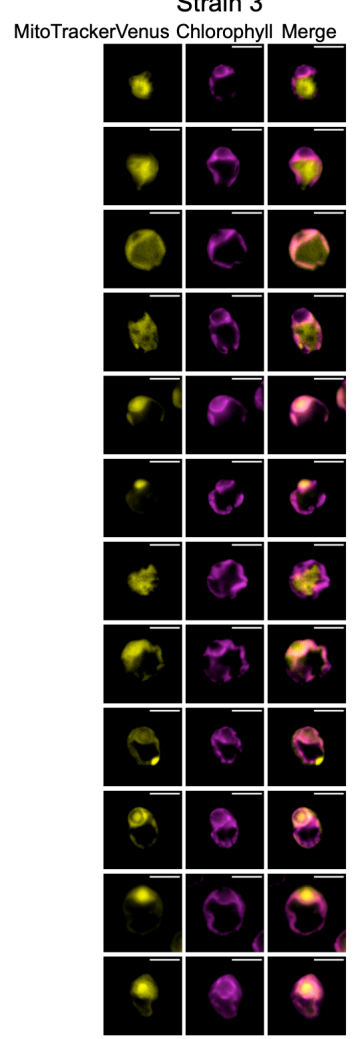

975 Supplementary Fig. 12. Microscopy for biological replicates of Bacillocin

976 1580. Three independent transformant lines (Strains 1-3) are shown for each

977 combination of modifications (A-L), represented by a cartoon and a shorthand

978 description (cf. Fig. 2). Where a construct was interpreted as generating reporter

979 localization in mitochondria or chloroplast, this is indicated by an orange ' $\mathrm{m}$ ' or a

980 green 'c' respectively, in bold for full targeting or in italics for partial targeting.

981 Scale bars are $5 \mu \mathrm{m}$. 


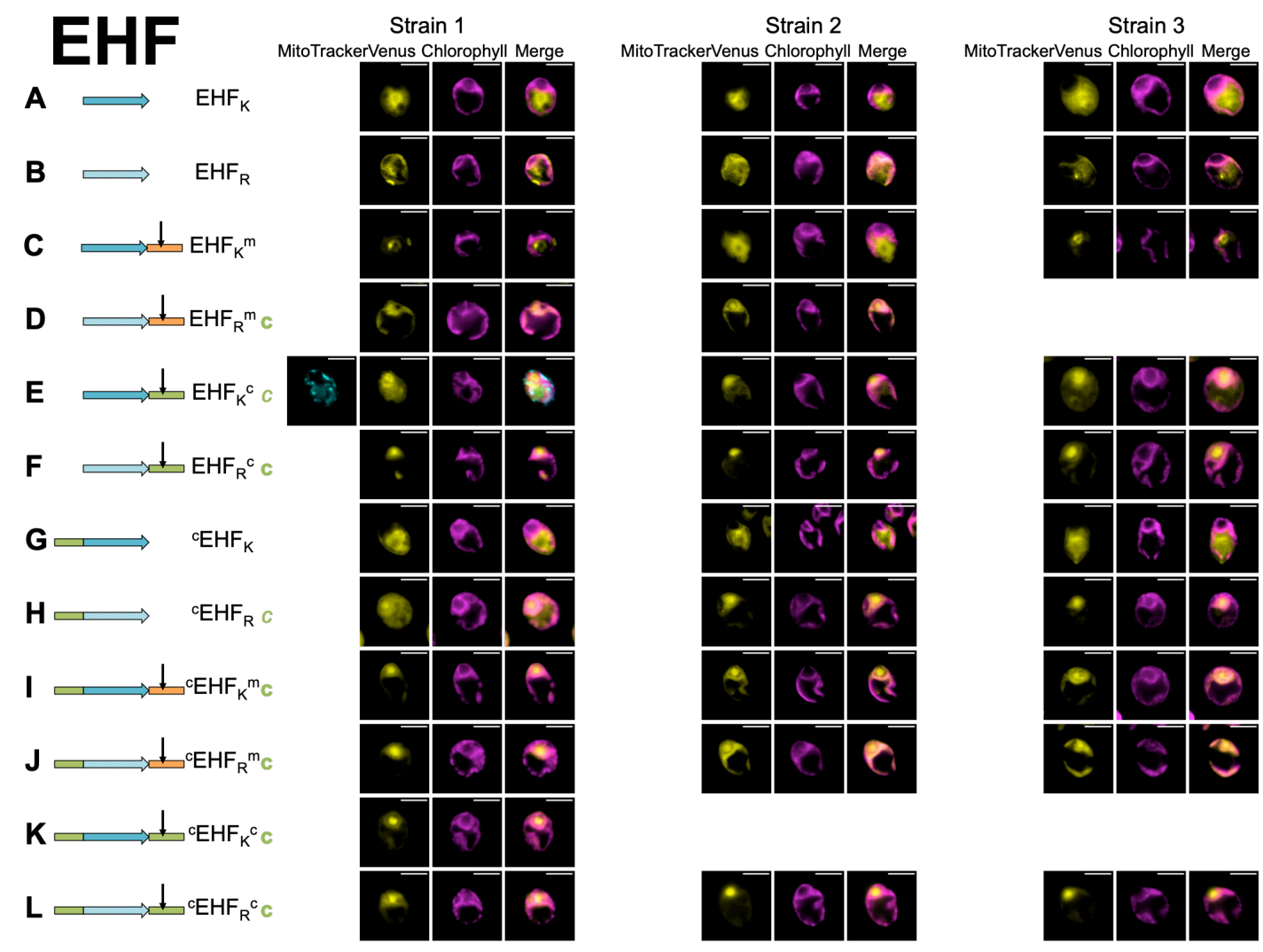

983 Supplementary Fig. 13. Microscopy for biological replicates of Enterocin

984 HF. Three independent transformant lines (Strains 1-3) are shown for each

985 combination of modifications (A-L), represented by a cartoon and a shorthand

986 description (cf. Fig. 2). Where a construct was interpreted as generating reporter

987 localization in mitochondria or chloroplast, this is indicated by an orange ' $\mathrm{m}$ ' or a

988 green ' $c$ ' respectively, in bold for full targeting or in italics for partial targeting.

989 Scale bars are $5 \mu \mathrm{m}$.

990 


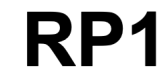

A

$\mathrm{B} \Longrightarrow \mathrm{RP}_{\mathrm{R}} \mathrm{m}$

C $\Longleftrightarrow \mathrm{RP}{ }^{\mathrm{c}}$

$\mathbf{D} \Longrightarrow{ }^{\mathrm{CRP}} 1_{\mathrm{R}}$<smiles></smiles>

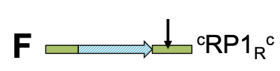

Strain 1

MitoTrackerVenus Chlorophyll Merge

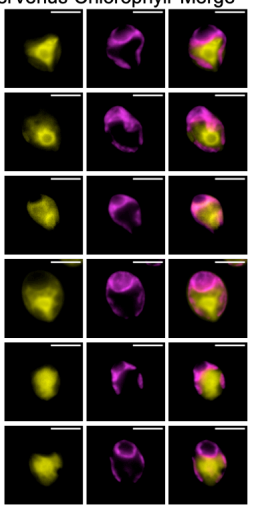

Strain 2

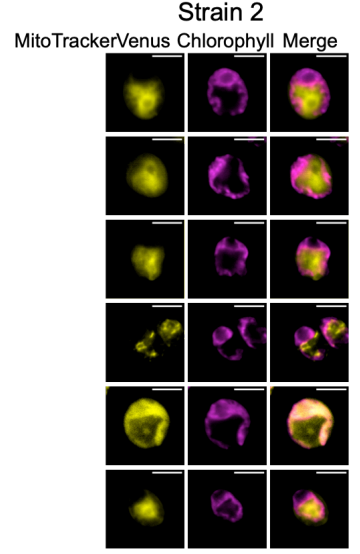

Strain 3

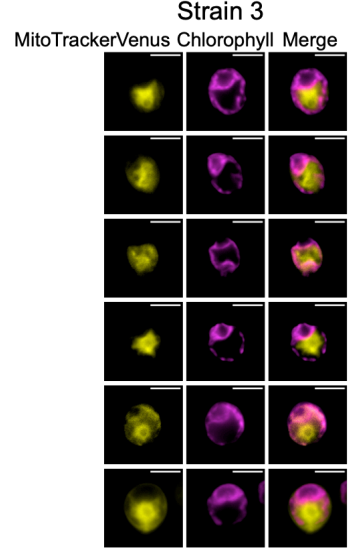

993 Supplementary Fig. 14. Microscopy for biological replicates of negative

994 control Random Peptide 1. Three independent transformant lines (Strains 1-3)

995 are shown for each combination of modifications (A-F), represented by a cartoon

996 and a shorthand description (cf. Fig. 2). Where a construct was interpreted as

997 generating reporter localization in mitochondria or chloroplast, this is indicated

998 by an orange ' $m$ ' or a green 'c' respectively, in bold for full targeting or in italics

999 for partial targeting. Scale bars are $5 \mu \mathrm{m}$. 


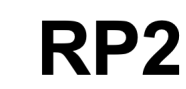

A

$\Rightarrow \mathrm{RP} 2_{\mathrm{K}}$

B $\Longrightarrow R P 2_{R}$

C $\Longleftrightarrow \mathrm{RP}_{\mathrm{K}} \mathrm{m}^{\mathrm{m}}$

D $\Longrightarrow \mathrm{RP}_{\mathrm{R}^{\mathrm{m}}}$

$E \rightleftharpoons \operatorname{RP} 2_{K}{ }^{c}$

$\mathbf{F} \longrightarrow \mathrm{RP} 2_{\mathrm{R}}^{\mathrm{c}}$

$\mathbf{G} \rightleftharpoons{ }^{\mathrm{cRP}} 2_{\mathrm{K}}$

$\mathbf{H} \rightleftharpoons{ }^{\mathrm{CRP}} \mathbf{2}_{\mathrm{R}}$

$\mathrm{I} \rightleftharpoons \mathrm{CRP}_{\mathrm{K}} \mathrm{m}$

$\mathbf{J} \rightleftharpoons \downarrow^{\mathrm{c} R P} 2_{\mathrm{R}}^{\mathrm{m}}$

$\mathbf{K} \rightleftharpoons \frac{1}{\mathrm{cRP} 2}{ }_{\mathrm{K}} \mathrm{c}$

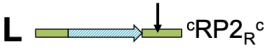

Strain 1

MitoTrackerVenus Chlorophyll Merge
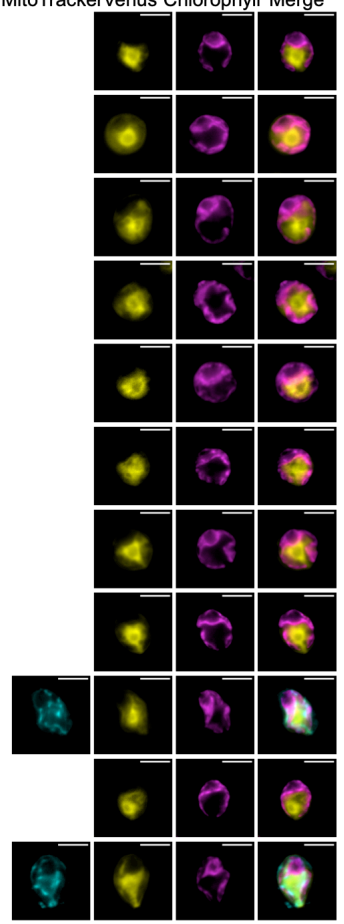

6
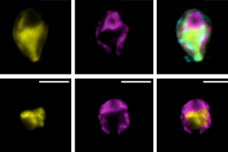

Strain 2

Mito TrackerVenus Chlorophyll Merge
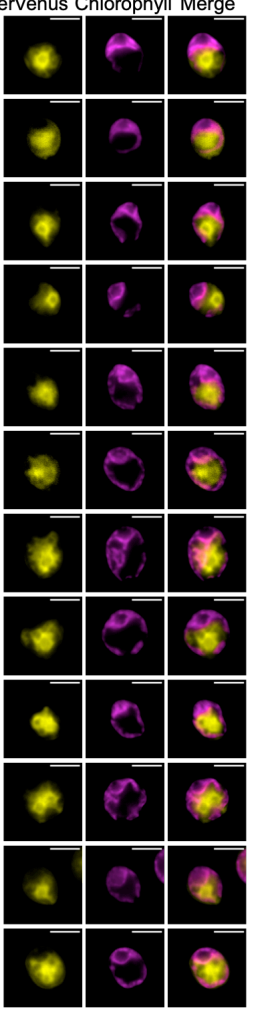

1001 Supplementary Fig. 15. Microscopy for biological replicates of negative

1002 control Random Peptide 2. Three independent transformant lines (Strains 1-3)

1003 are shown for each combination of modifications (A-L), represented by a cartoon

1004 and a shorthand description (cf. Fig. 2). Where a construct was interpreted as

1005 generating reporter localization in mitochondria or chloroplast, this is indicated

1006 by an orange ' $m$ ' or a green ' $c$ ' respectively, in bold for full targeting or in italics

1007 for partial targeting. Scale bars are $5 \mu \mathrm{m}$. 

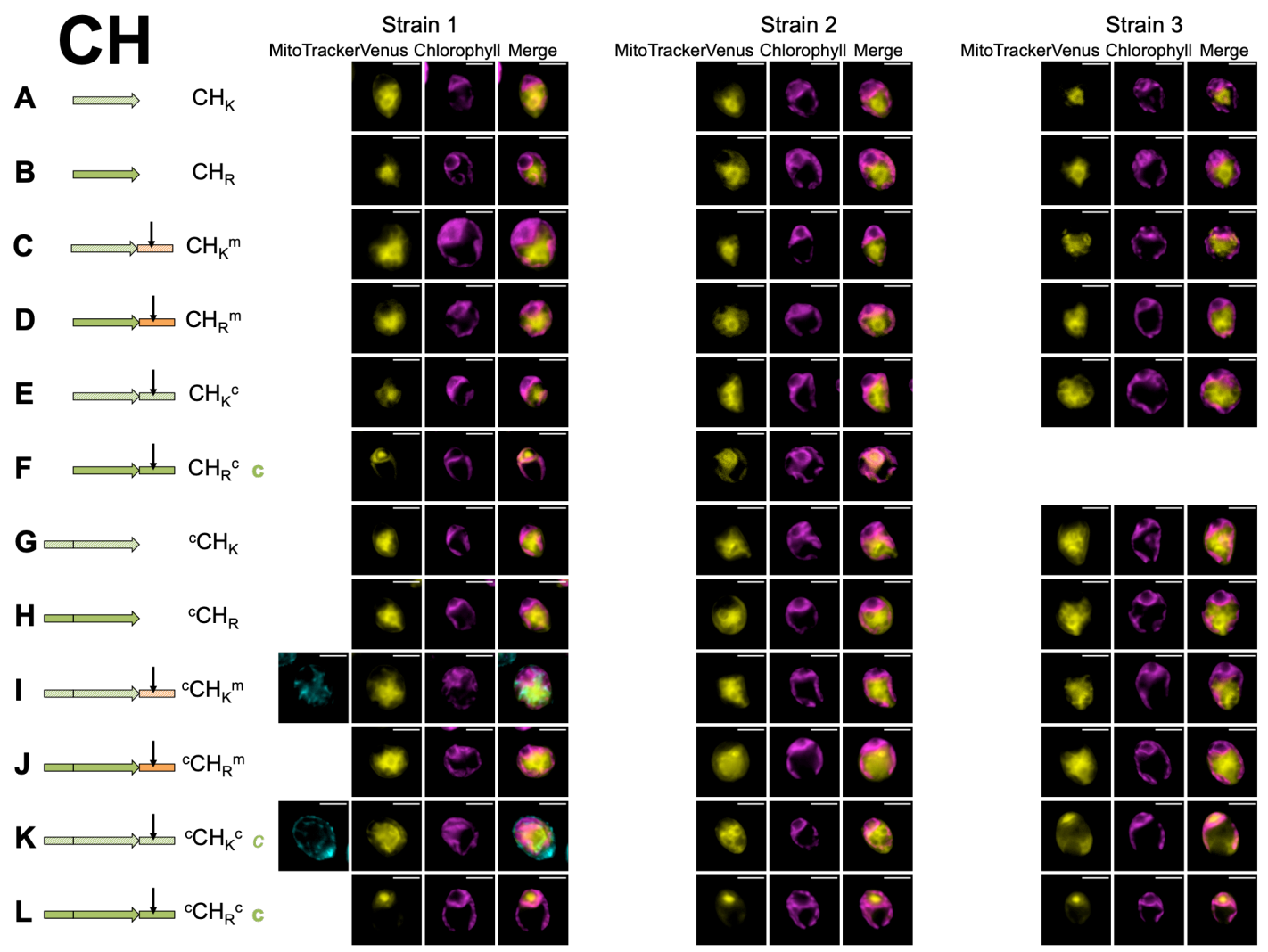

1009 Supplementary Fig. 16. Microscopy for biological replicates of TP control

1010 Rubisco activase cTP helical element (CH). Three independent transformant

1011 lines (Strains 1-3) are shown for each combination of modifications (A-L),

1012 represented by a cartoon and a shorthand description (cf. Fig. 2). Where a

1013 construct was interpreted as generating reporter localization in mitochondria or

1014 chloroplast, this is indicated by an orange ' $m$ ' or a green ' $c$ ' respectively, in bold

1015 for full targeting or in italics for partial targeting. Scale bars are $5 \mu \mathrm{m}$. 


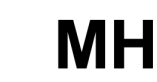

A $\rightleftharpoons \mathrm{MH}_{\mathrm{K}}$

B $\Longrightarrow \mathrm{MH}_{\mathrm{R}}$

C $\Longrightarrow \mathrm{MH}_{\mathrm{K}}{ }^{\mathrm{m}}$

D $\Longleftrightarrow \mathrm{MH}_{\mathrm{R}} \mathrm{m}^{m}$

E $\Longrightarrow \mathrm{MH}_{\mathrm{K}} \mathrm{C}^{\mathrm{Cr}}$

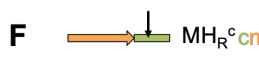

$\mathbf{G} \rightleftharpoons{ }^{\mathrm{c}} \rightleftharpoons \mathrm{H}_{\mathrm{K}}$

$\mathbf{H} \rightleftharpoons{ }^{\mathrm{c}} \mathrm{MH}_{\mathrm{R}}$

$\mathrm{I} \rightleftharpoons \mathrm{cMH}_{\mathrm{K}}{ }^{\mathrm{m}}$

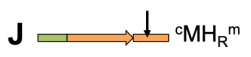

$\mathbf{K} \Longrightarrow \mathrm{CMH}_{\mathrm{K}}{ }^{\mathrm{c}} \mathrm{c}$

$\mathbf{L} \Longrightarrow \mathrm{MH}_{\mathrm{R}}{ }^{\mathrm{C}} \mathrm{c}$
Strain 1
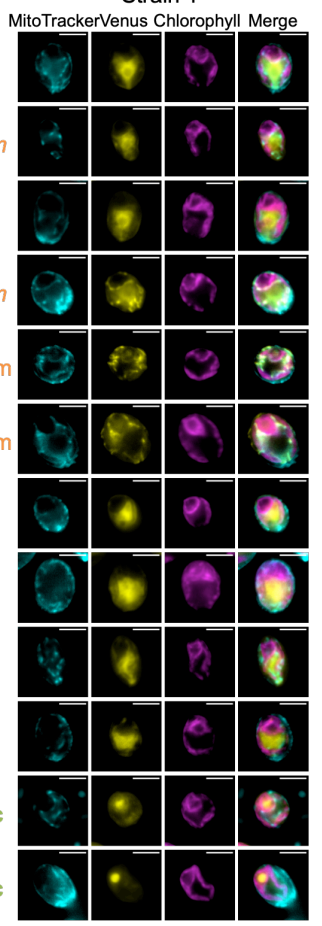

Strain 2
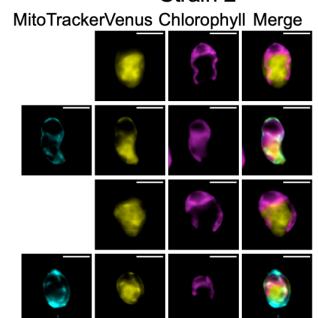

3
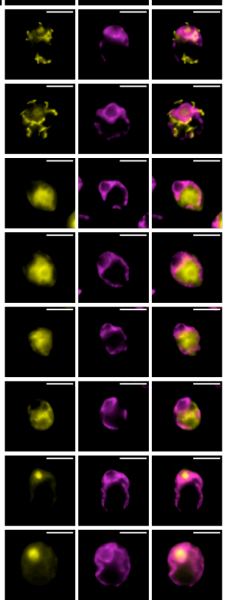

Strain 3

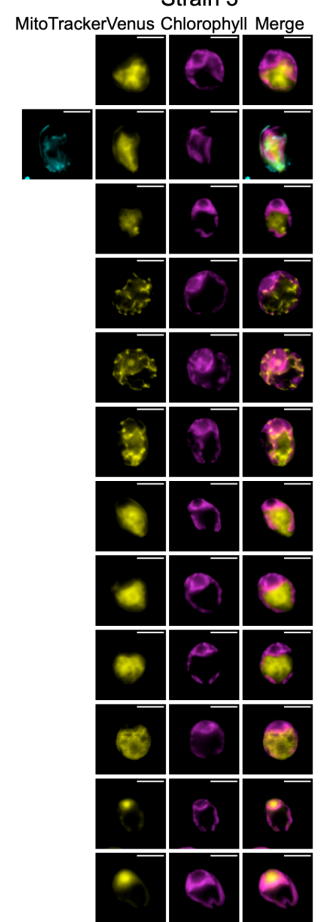

1017 Supplementary Fig. 17. Microscopy for biological replicates of TP control

1018 mitochondrial $\boldsymbol{\gamma}$-carbonic anhydrase 2 mTP helical element (MH). Three

1019 independent transformant lines (Strains 1-3) are shown for each combination of

1020 modifications (A-L), represented by a cartoon and a shorthand description (cf.

1021 Fig. 2). Where a construct was interpreted as generating reporter localization in

1022 mitochondria or chloroplast, this is indicated by an orange ' $m$ ' or a green ' $c$ '

1023 respectively in bold for full targeting or in italics for partial targeting. Scale bars

1024 are $5 \mu \mathrm{m}$. 

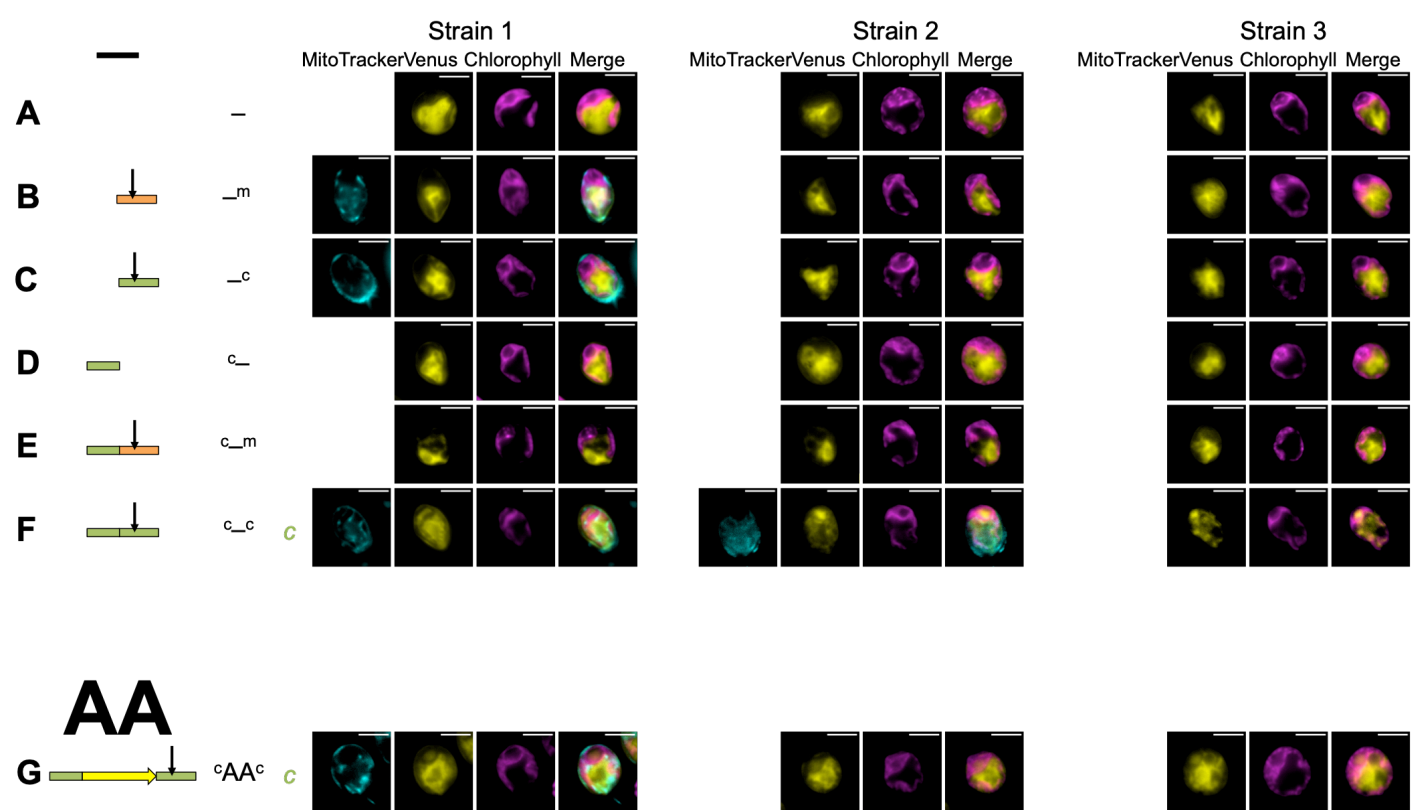

1027 and Alanine-screen controls. Three independent transformant lines (Strains 1-

1028 3) are shown for each combination of elements (A-G), represented by a cartoon

1029 and a shorthand description (cf. Fig. 2). Where a construct was interpreted as

1030 generating reporter localization in mitochondria or chloroplast, this is indicated

1031 by an orange ' $m$ ' or a green ' $c$ ' respectively, in bold for full targeting or in italics

1032 for partial targeting. Scale bars are $5 \mu \mathrm{m}$.

1033 


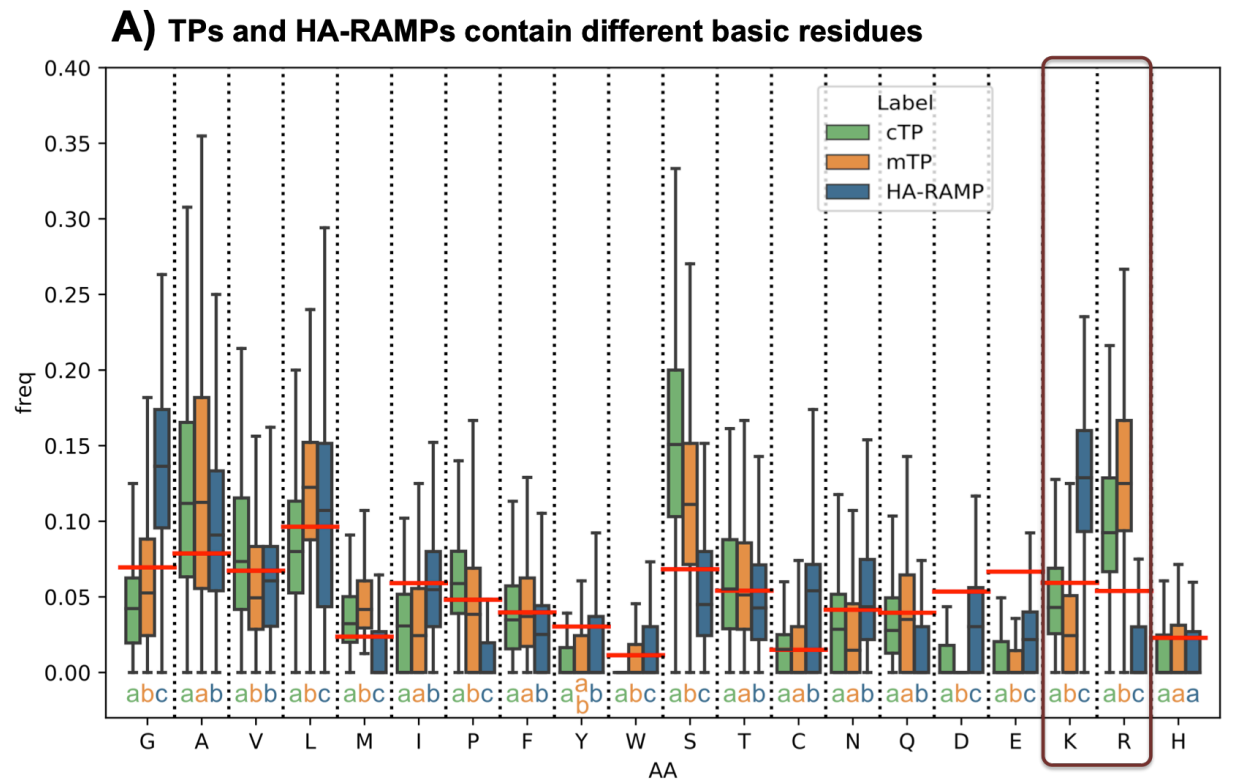

1036 Supplementary Fig. 19. Comparison of amino acid frequencies reveals K/R

1037 shift. A) Amino acid frequencies are shown as boxplots (center line: median; box 1038 limits: upper and lower quartiles; whiskers: $\min / \max$ values within $1.5 \mathrm{x}$ 1039 interquartile range) for Chlamydomonas cTPs in green, Chlamydomonas mTPs in 1040 orange and HA-RAMPs in blue. To give a baseline for comparison, the average 1041 across uniprot is given for each amino acid as red horizontal line. For each 1042 residue, different letters underneath distributions indicate a significant 1043 difference: groups that share the same letter are significantly different at $\mathrm{p}<0.05$ 1044 (Multiple Kruskal Wallis tests followed by Dunn post-hoc tests) from groups 1045 attributed a different letter. 


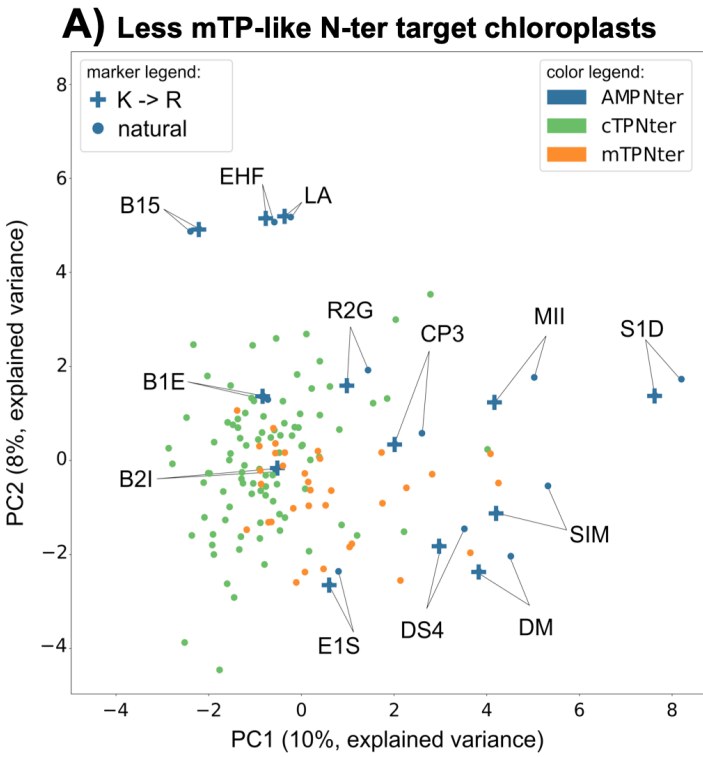

\section{B) More TP-like C-ter enable targeting}

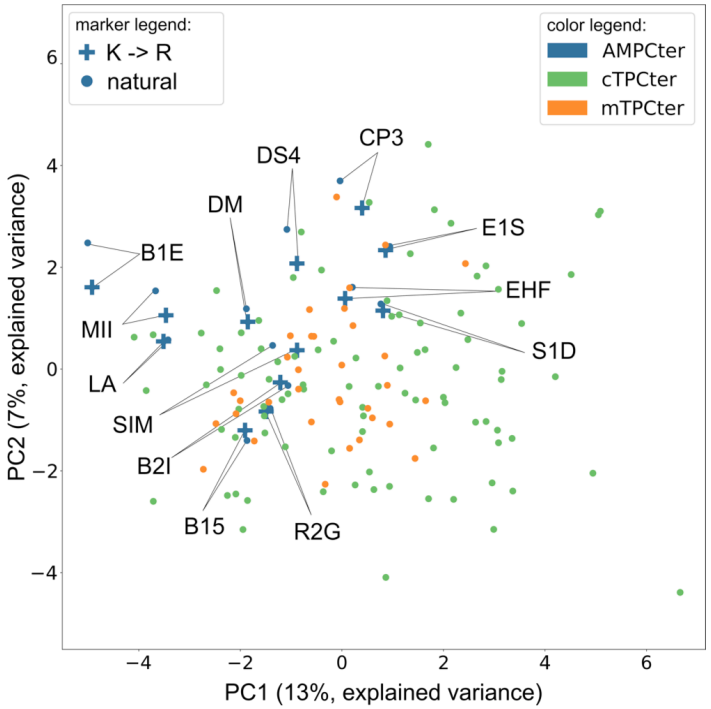

1049 Supplementary Fig. 20. Principal component analyses reveal that $\mathrm{N}$ - but not

1050 C-termini differ between cTPs and mTPs. Principal component (PC) analyses

1051 of auto-cross-correlated Z-scale values ${ }^{5}$ for A) N-termini (15 residues) and B) C-

1052 termini (33 residues, encompassing -10 to +23 relative to the cleavage site for

1053 TPs) respectively of Chlamydomonas cTPs (in green) and mTPs (in orange) as

1054 well as the 13 HA-RAMPs under study (in blue; crosses denote HA-RAMPs after $1055 \mathrm{~K} \rightarrow \mathrm{R})$. 
A) cTP charge distribution

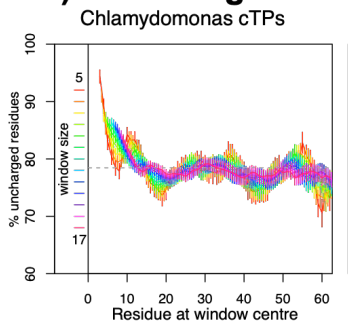

C) Arginines in N-ter

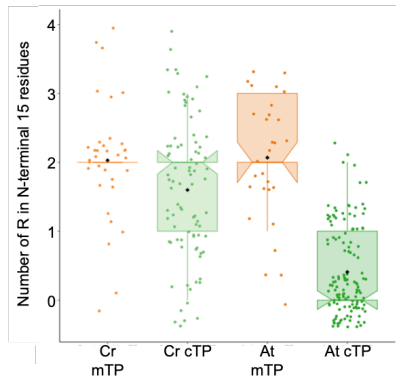

F) C. reinhardtii cTP and mTP N-ter differ
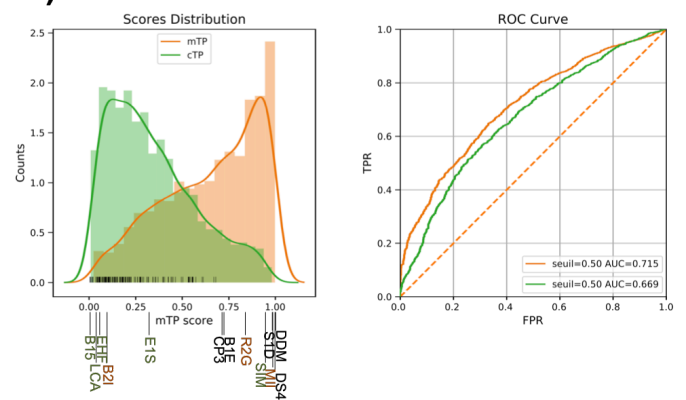

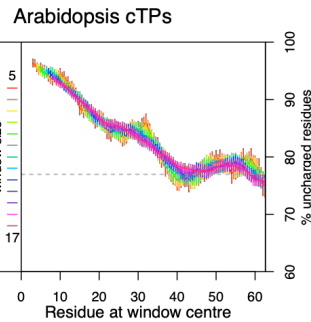

D) N-ter hydrophobicity

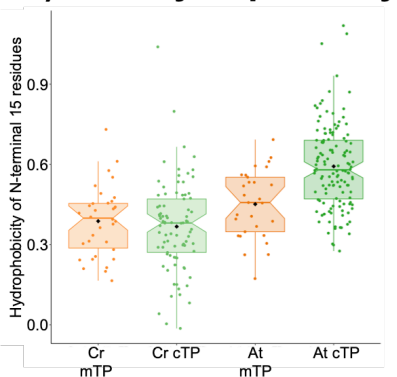

B) mTP charge distribution

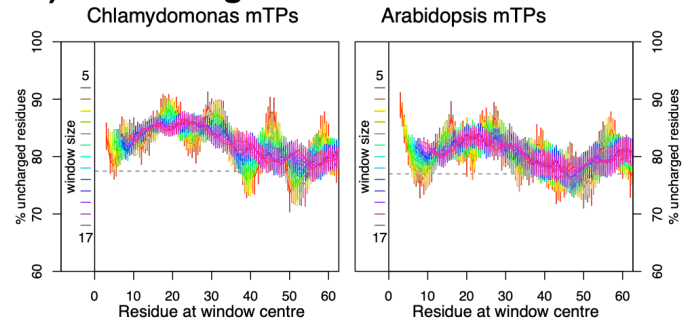

E) N-ter disorder

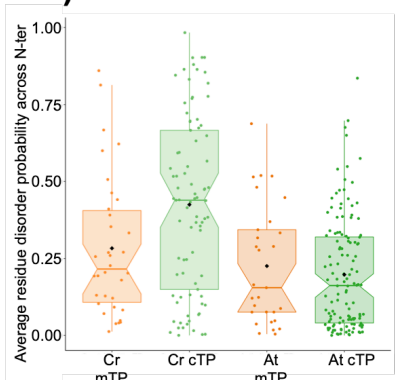

G) A. thaliana cTP and mTP N-ter differ
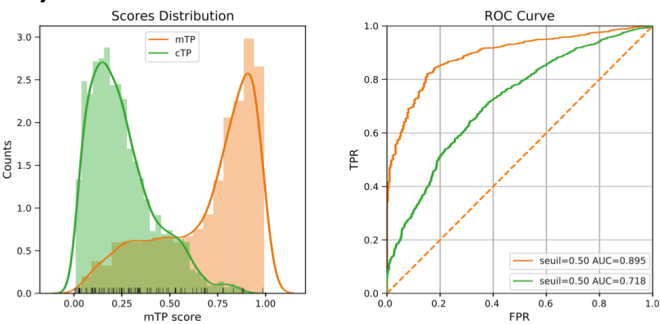

Supplementary Fig. 21. Unusual algal cTP N-termini share physicochemical differences against mTP N-termini with plant counterparts. A) Plots

1059 detailing the percentage of uncharged residues ${ }^{25}$ (i.e. excluding K/R and D/E) for

1060 window sizes ranging from 5 to 17 residues (refer to legend for colour code)

1061 show that Chlamydomonas cTPs (on the left) are less uncharged at the N1062 terminus than Arabidopsis cTPs (on the right). Dashed grey lines indicate the 1063 average value for randomized sequences (including mature proteins), to provide 1064 an estimate of what would be expected by chance. B) Equivalent graphs (as in A) 1065 for mTPs show a similar charge profile across Chlamydomonas and Arabidopsis. 1066 C-E) Distributions of salient values as boxplots (center line: median; box limits: upper and lower quartiles; whiskers: $\min / \max$ values within $1.5 x$ interquartile 
range) for mTPs in orange and cTPs in green with Chlamydomonas reinhardtii

(Cr) on the left and Arabidopsis thaliana (At) on the right show that: C)

1070 Chlamydomonas cTP N-termini contain R at almost the same frequency as mTP

1071 N-termini, whereas Arabidopsis cTP N-termini contain fewer R; D)

1072 Chlamydomonas TP N-termini are less hydrophobic than Arabidopsis TP N-

1073 termini, with plant cTP N-termini showing the highest hydrophobicity; E)

1074 Chlamydomonas cTP N-termini are more disordered than mTP or Arabidopsis

1075 cTP N-termini. Points represent individual peptides (note that for integer values,

1076 point positions are randomized within \pm 0.5 in $\mathrm{y}$ as well as in $\mathrm{x}$ to increase point

1077 separation), population means are shown as black diamonds. F) Binomial logistic

1078 regression classifier separating TPs using auto-cross-covariance of Z-scales ${ }^{5}$ of

1079 the N-terminal 15 residues for $C$. reinhardtii. The left-hand graph shows the

1080 distribution of cTPs in green and mTPs in orange over the model output 'mTP

1081 score'. Black bars at the bottom of the graph represent scores for cTPs of

1082 Arabidopsis, showing that plant cTPs are recognized as cTPs by the model

1083 trained on algal cTP N-termini (89\% recognized as cTPs, with an mTP score

$1084<0.5)$. Values for our 13 HA-RAMPs are given below the graph. The right-hand

1085 graph shows receiver operating characteristic (ROC) curves, plotting the true 1086 positive rate (TPR) against the false positive rate (FPR), where the area under

1087 the curve (AUC) serves as estimate of the model quality with values above 0.5 1088 indicating that the model is better than random. G) Equivalent model (as in F) 1089 trained on $A$. thaliana TPs, with black bars now representing Chlamydomonas 1090 cTPs showing that the majority of algal cTPs are recognized as cTPs by the plant 1091 model $(75 \%$ recognized as cTPs, with an mTP score $<0.5)$. 
A) $K \rightarrow R$ increases HA-RAMP targeting HA-RAMP constructs

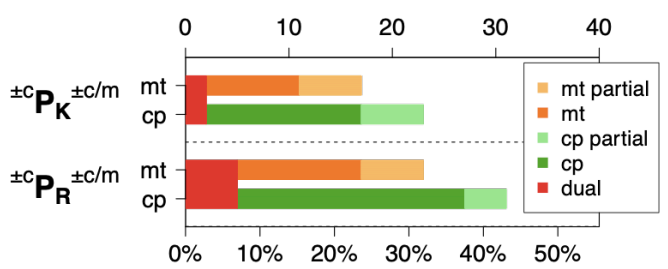

C) $\mathrm{K} \rightarrow \mathrm{R}$ can ameliorate targeting MitoTracker Venus Chlorophyll Merge
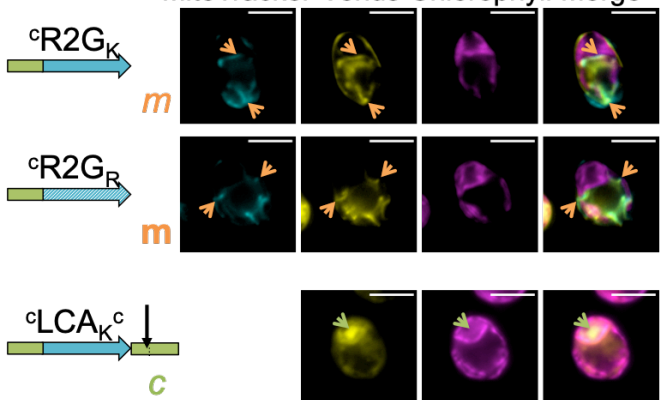

$$
\mathrm{CLCA}_{\mathrm{R}}^{\mathrm{C}}
$$

c

$$
\underset{c}{\mathrm{LLCA} A_{\mathrm{R}}^{\mathrm{m}}}
$$
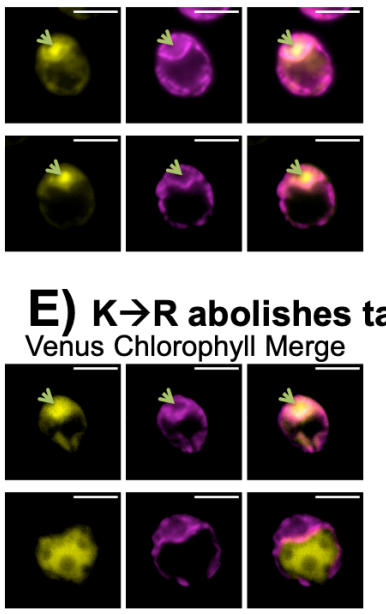

B) $K \rightarrow R$ can enable targeting

MitoTracker Venus Chlorophyll Merge
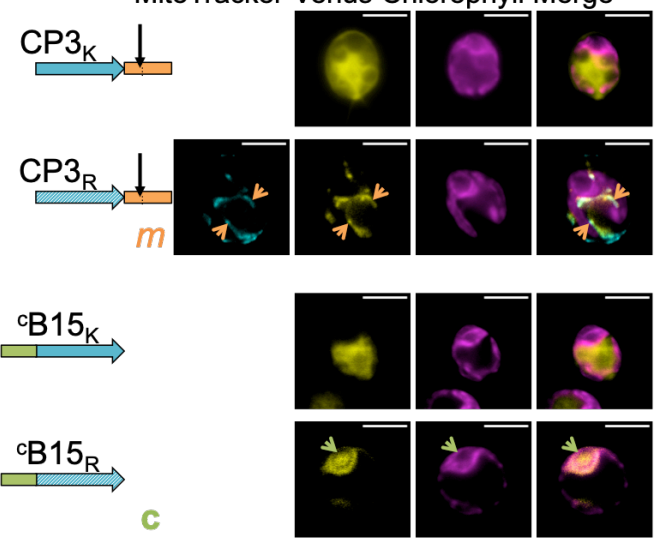

D) $K \rightarrow R$ can generate dual targeting MitoTracker Venus Chlorophyll Merge

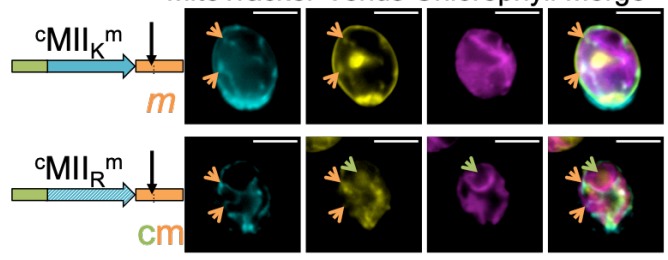

E) $K \rightarrow R$ abolishes targeting in $\mathbf{2}$ instances
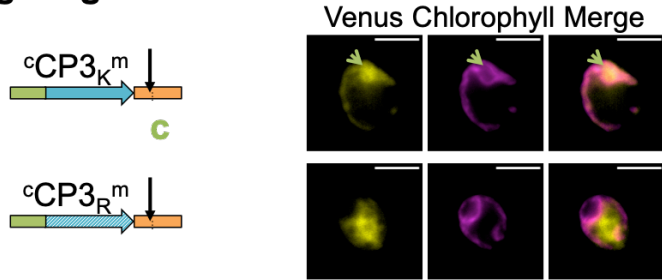

1093 Supplementary Fig. 22. K $\rightarrow$ R generally improves HA-RAMP targeting. A) An

1094 overview graph showing how many, out of 13 HA-RAMPs studied, show

1095 targeting to either mitochondria (mt) or chloroplast (cp), or both (dual) before

1096 and after $\mathrm{K} \rightarrow \mathrm{R}$ represented. B-E) Epifluorescence microscopy images of selected

1097 examples are shown as in Fig. 3; same convention for Venus localization as in Fig.

10984. 


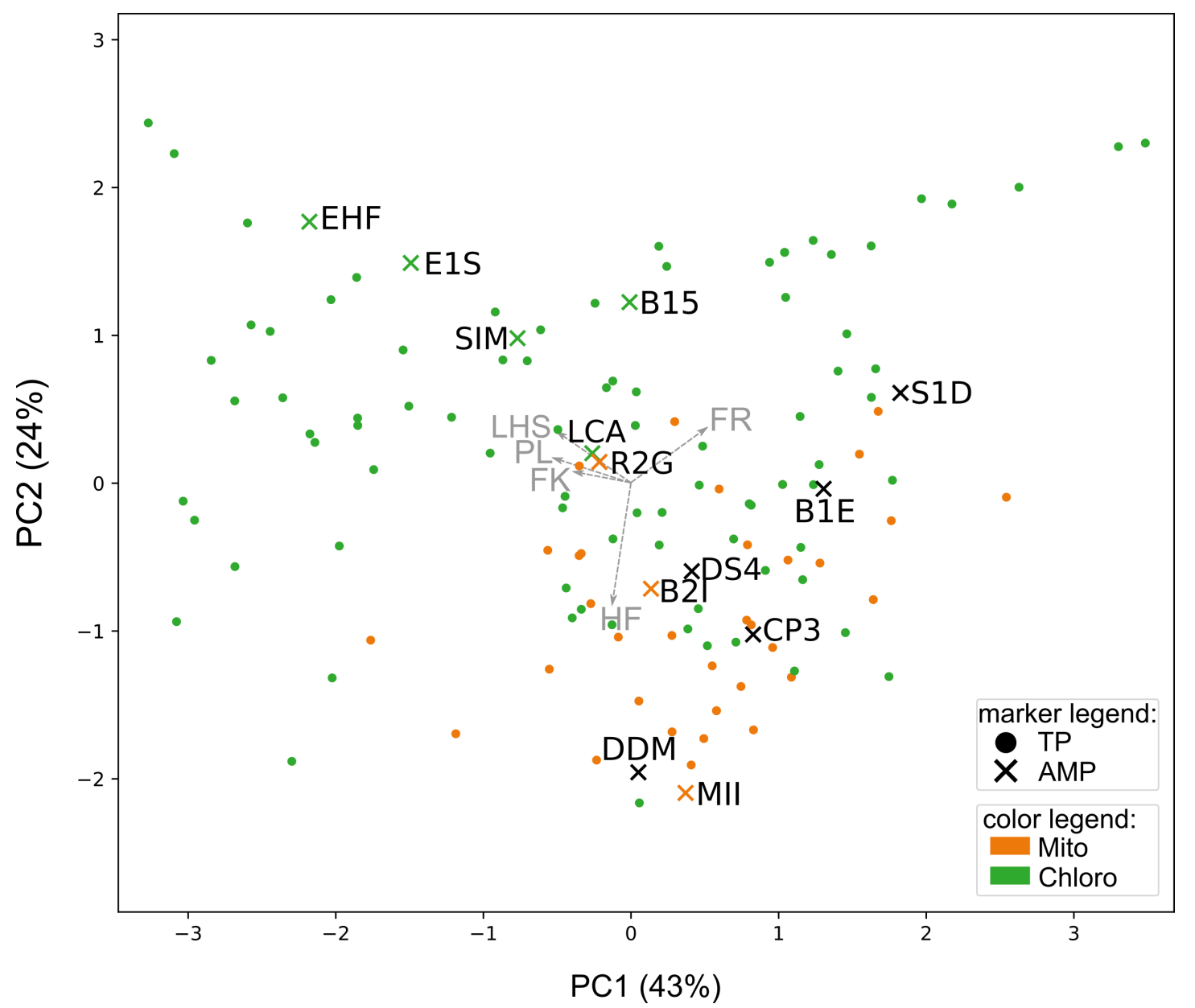

1101 Supplementary Fig. 23. HA-RAMP properties determine their targeting

1102 propensities. PCA analysis of cTPs (green dots), mTPs (orange dots), cp-set HA-

1103 RAMPs (green crosses), mt-set HA-RAMPs (orange crosses) and other HA-

1104 RAMPs (dark crosses) based on their length (Peptide Length: PL), number of

1105 residues before the longest predicted amphipathic helix (Long Helix Start: LHS),

1106 fraction of residues forming the predicted amphipathic helix (Helix Fraction: HF)

1107 and fraction of $\mathrm{K}$ (Lysine Fraction: KF) and R residues (Arginine Fraction: AF).

1108 Arrows on the graph represent the eigenvalues of the individual variables. The

1109 first principal component explains $43 \%$ of the variance and distinguishes

1110 peptides principally according to KF, PL, LHS (pointing left) and RF residues

1111 (pointing right). The second principal component explains $24 \%$ of the variance

1112 and distinguishes peptides principally according to HF. The cp-set HA-RAMPs 
1113 group with the most distinguishable cTPs, being longer, with a higher LHS and a

1114 lower HF compared to mt-set HA-RAMPs and mTPs.

A) Interactivity: Boman

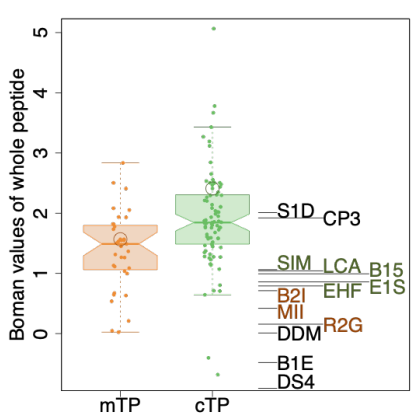

D) FGLK in Arabidopsis
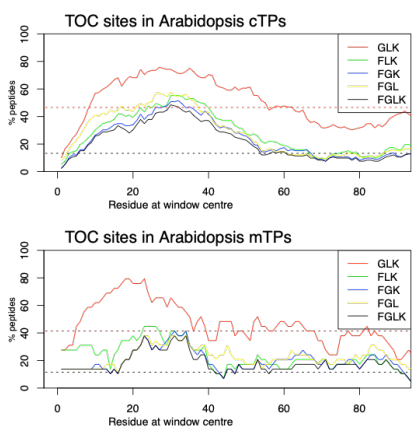

B) Interactivity: Anchor2

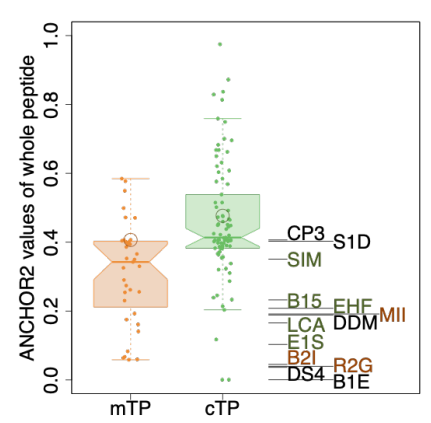

E) FGLK in Chlamydomonas
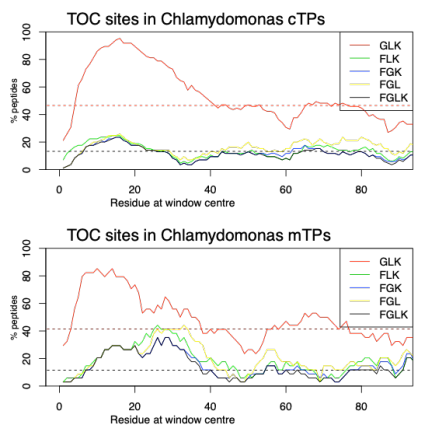

C) putative Hsp70 sites
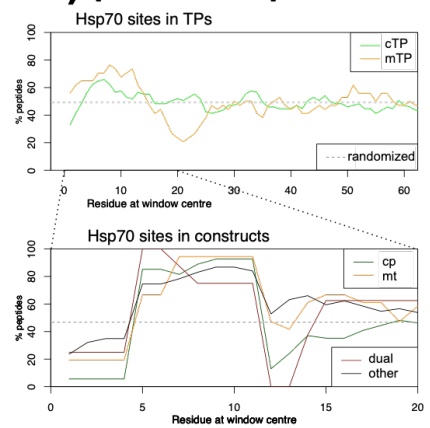

1116 Supplementary Fig. 24. Higher protein interactivity predicted for cTPs than

1117 mTPs. A, B and F) For salient properties, Chlamydomonas mTPs and cTPs are

1118 compared to HA-RAMPs (cp-set in green, mt-set in orange). Distributions are

1119 shown as boxplots (center line: median; box limits: upper and lower quartiles;

1120 whiskers: $\min / \max$ values within $1.5 \mathrm{x}$ interquartile range), coloured points

1121 represent individual peptides A) cTPs show increased protein interactivity $1122(\mathrm{p}=0.0003)$, as do cp-set HA-RAMPs $(\mathrm{p}=0.0355)$, as estimated through Boman 1123 values ${ }^{65}$, a proxy developed for AMPs where a value of ca. $>2$ indicates increased

1124 protein interaction potential, and $<2$ points to membrane interaction. B) cTPs 1125 show increased protein interactivity $(\mathrm{p}<0.0001)$, and $\mathrm{cp}$-set HA-RAMPs show a 1126 similar tendency ( $\mathrm{p}=0.0638)$, as estimated with Anchor $2^{67}$, developed to predict 
1127 the protein interaction potential of disordered sequences. C) Both cTPs and

1128 mTPs show a high fraction of peptides $(\sim 50 \%)$ that have an Hsp70 interaction

1129 site over a 6-residue window at any given position along the sequence in the top

1130 graph, with a peak towards the N-terminus. HA-RAMP constructs shown in the

1131 bottom graph also display a peak in predicted Hsp70-binding sites at the N-

1132 terminus, independently of construct localisation. The value obtained after

1133 randomizing the position of residues is given as dashed grey line to provide an

1134 estimate of how often sites would be expected to occur given amino acid

1135 frequencies ( $\sim 50 \%)$. Randomization was done over the entire sequence

1136 including the cargo protein. D) For Arabidopsis cTPs in the top graph, the

1137 percentage of peptides that have a full 'FGLK' site ${ }^{25}$ (black curve; presence of F

1138 and G/P and L/V/A and K/R and absence of D/E within an 8-residue window)

1139 far exceeds the value obtained after randomizing the position of residues

1140 (dashed black line for full 'FGLK' sites), a proxy for the frequency of the motif

1141 expected at random, for most positions along the sequence up to $\sim 60$ residues.

1142 Randomization was done over the entire sequence including the cargo protein.

1143 Reduced 'FGLK'-1 sites containing three out of four elements appear to occur

1144 mostly in the context of full FGLK sites, except for 'GLK' sites for which

1145 randomization is also shown (dashed red line). Arabidopsis mTPs (bottom

1146 graph) also contain 'FLGK' sites at a frequency higher than expected at random

1147 between residues 20-40, and 'GLK' sites upstream of residue 40, but motifs are

1148 less prevalent than in cTPs. E) Equivalent graphs (as D) for Chlamydomonas

1149 show that full 'FGLK' sites (black lines) are rare in cTPs (top graph) and actually

1150 more common in mTPs (bottom graph) upstream of residue 40. Reduced 'GLK'

1151 sites (red lines) by contrast far exceed the prevalence expected at random 
1152 (dashed red line) ca. up to residue 40. F) Protein interactivity, estimated through

1153 Boman values, for only those parts of peptides that correspond to FGLK-1 sites is

1154 increased for cTPs $(\mathrm{p}<0.0001)$, and $\mathrm{cp}$-set HA-RAMPs show a similar tendency

$1155(\mathrm{p}=0.0627)$. Note that cTP-C and cTP-N, but not mTP-C, contain 'FGLK-1' sites

1156 with predicted protein interactivity. *DS4 contains no 'FGLK-1' sites. Reported p-

1157 values were obtained through two-way t-tests for TPs and one-way t-tests for

1158 HA-RAMPs.

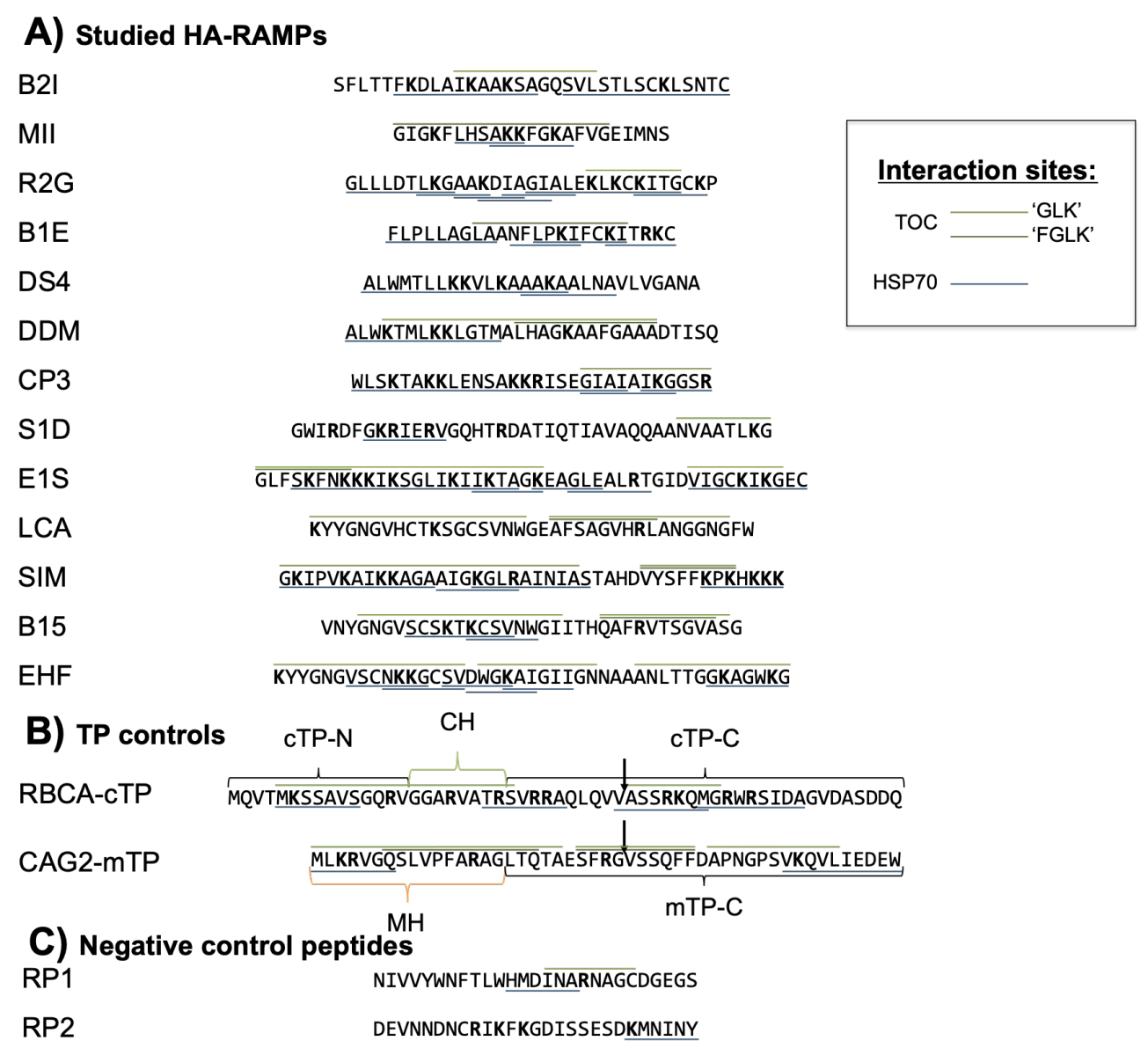

1160 Supplementary Fig. 25. Hsp70 and (F)GLK sites are present in HA-RAMPs.

1161 Predicted Hsp70 binding sites are underlined in blue. Putative TOC interaction

1162 sites are overlined in green: 'FGLK' sites in dark green and 'GLK' sites in light

1163 green. See Fig. 1 for detailed annotations. Notably, both TOC and Hsp70- 
1164 interaction sites are contained within native HA-RAMPs (A) and within our TP

1165 controls (B).

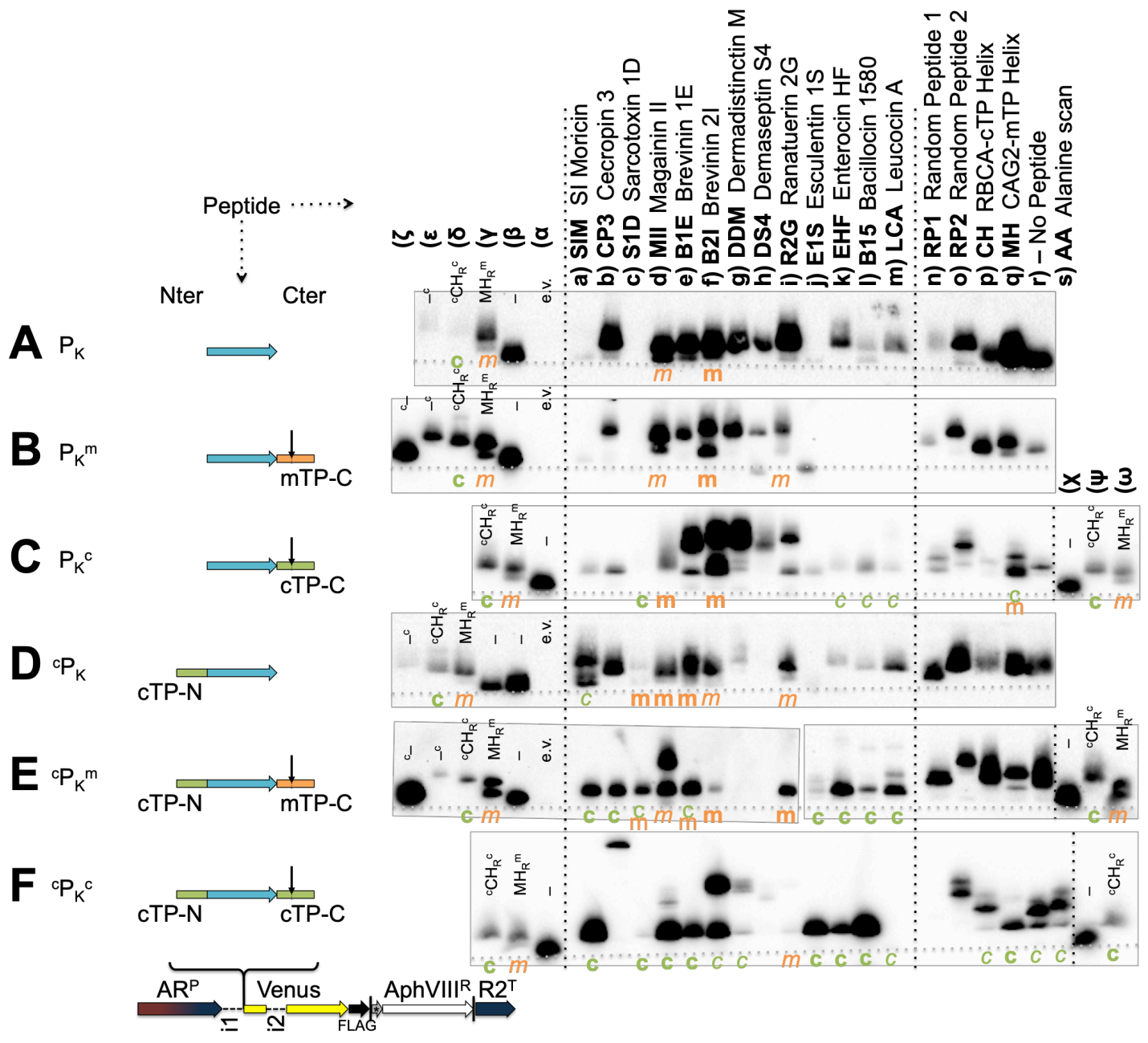

1167 Supplementary Fig. 26. Western Blots for K-bearing constructs. Samples

1168 were immunolabelled using an $\alpha$-FLAG primary antibody. Constructs

1169 representing different combinations of TP-element additions are shown in A-F,

1170 as indicated by a shorthand description and a cartoon (cf. Fig. 2). HA-RAMPs are

1171 consistently arranged in lanes a-m. Note that the order of HA-RAMPs differs from

1172 the one in Fig. 2. Control constructs in lanes o-r carry the $\mathrm{R} \rightarrow \mathrm{K}$ modification.

1173 Additional technical controls are present in lanes labeled with greek letters.

1174 Sections containing HA-RAMPs, control constructs and technical controls are 1175 separated by dotted black lines. Technical controls (cf. Fig. 1): - is no peptide, 


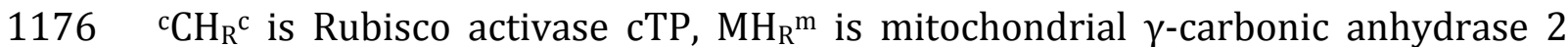

1177 mTP, c- is Rubisco activase cTP N-terminal element (15 residues), ${ }^{-c}$ is Rubisco

1178 activase cTP $\mathrm{C}$-terminal element (33 residues, -10 to +23 relative to cleavage

1179 site), e.v. is empty vector (no Venus expression). Constructs that generated

1180 chloroplast and/or mitochondrial localization are marked with a green 'c' and/or

1181 an orange ' $m$ ' respectively, in bold for full or in italics for partial targeting. Note

1182 that E) contains two separate blots for the left-hand section up to lane $\mathrm{i}$ and the

1183 right-hand section following lane $\mathrm{j}$. 